\title{
Electron transport in disordered Luttinger liquid
}

\author{
I.V. Gornyi ${ }^{1, *}$, A.D. Mirlin ${ }^{1,2, \dagger}$, and D.G. Polyakov ${ }^{1, *}$ \\ ${ }^{1}$ Institut für Nanotechnologie, Forschungszentrum Karlsruhe, 76021 Karlsruhe, Germany \\ ${ }^{2}$ Institut für Theorie der kondensierten Materie, Universität Karlsruhe, 76128 Karlsruhe, Germany
}

(Dated: July 14, 2021)

\begin{abstract}
We study the transport properties of interacting electrons in a disordered quantum wire within the framework of the Luttinger liquid model. We demonstrate that the notion of weak localization is applicable to the strongly correlated one-dimensional electron system. Two alternative approaches to the problem are developed, both combining fermionic and bosonic treatment of the underlying physics. We calculate the relevant dephasing rate, which for spinless electrons is governed by the interplay of electron-electron interaction and disorder, thus vanishing in the clean limit. Our approach provides a framework for a systematic study of mesoscopic effects in strongly correlated electron systems.

PACS numbers: 71.10.Pm, 73.21.-b, 73.63.-b, 73.20.Jc
\end{abstract}

\section{INTRODUCTION}

Mesoscopics of strongly correlated electron systems has emerged as an area of great interest to both experimental and theoretical communities working in the field of nanoscale physics. Recently, progress in manufacturing of nanodevices has paved the way for systematic transport measurements on narrow quantum wires with a few or single conducting channels. Most prominent examples of these are: single-wall carbon

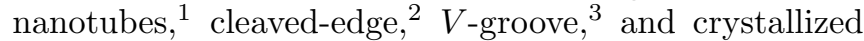
in a matrix ${ }^{4}$ semiconductor quantum wires, quantum Hall edges running in opposite directions and interconnected by means of tunneling,,$\underline{\underline{5}, 6}$ polymer nanofibers, ${ }^{\frac{7}{7}}$ and metallic nanowires,$\underline{8,9}$

Much experimental attention has been focused on the electrical properties of single-wall nanotubes (Refs 10,11, 12, 13, 14, 15, 16, 17, 18, 19, 20, 21, 22, 23, 24, 25 and references therein). In particular, evidence has emerged pointing towards the existence of Luttinger liquid in metallic singlewall carbon nanotubes, as expected for strongly interacting electrons in one dimension (1d). The Luttinger liquid behavior was observed via the power-law temperature and bias-voltage dependence of the current through tunneling contacts attached to the nanotubes. In the past few years, technological advances have made possible the fabrication of contacts between nanotubes and metallic leads with a very low contact resistance, which allows one to observe mesoscopic effects, $\frac{19,20,21,22}{1,}$ Further, techniques to grow very long (of the $\mathrm{mm}$ scale) nanotubes have been developed and the corresponding transport measurements performed $\underline{26}$

On the theoretical side, the challenge is to expand the ideas that have been developed for mesoscopic disordered systems on one side and for strongly correlated clean systems on the other. The situation is particularly interesting in $1 \mathrm{~d}$, where both disorder and electronelectron (e-e) interaction, even if they are weak, modify dramatically the large-scale, low-energy physics of the problem. In a clean wire, e-e interaction leads to the formation of a Luttinger-liquid ground state. The prop- erties of the Luttinger liquid without impurities and in the presence of a single barrier are known in great detail, see Refs. 27, 28, 29,30,31,32 for review. Much less is known about the Luttinger liquid in the presence of many impurities (disordered Luttinger liquids). The influential works by Apel and Rice ${ }^{33}$ and by Giamarchi and Schulz ${ }^{34}$ have defined the state of the art in this area for two decades. Recent years have seen a revival of interest in disordered Luttinger liquids, $, 35,36,37,38,39,40,41,42,43$ largely motivated by the technological advances mentioned above. However, the very applicability of such key notions of the mesoscopic physics as weak localization (WL) and dephasing to a disordered 1d system, not to mention a detailed analysis of these effects, remained an open problem. This problem is a subject of the present paper. Throughout the paper we study spinless (spinpolarized) electrons. Spin-related effects will be considered in Ref. 44.

The structure of the paper is as follows. In Sec. II we summarize the earlier achievements in the mesoscopics of higher-dimensional systems on one side and in the theory of strongly correlated 1d systems on the other. Section III contains an exact formulation of the problem, as well as a discussion of our strategy in solving it. In Sec. [IV] we present the bosonization method and the renormalization-group technique that we use to account for the Luttinger-liquid renormalizations. Having analyzed in Sec. $\mathrm{V}$ the e-e inelastic scattering rate in $1 \mathrm{~d}$, we turn to the calculation of a weak-localization correction to the conductivity and the associated dephasing rate in Sec. VI] We also compare this dephasing rate with the one that governs the damping of AharonovBohm oscillations. Section VII is devoted to the analysis of the transport properties of a disorder Luttinger liquid by using an alternative approach - the "functional bosonization" - which allows one to treat the renormalization and the inelastic scattering on an equal footing. Our results are summarized in Sec. VIII Technical details of the calculations are presented in several Appendices. Some of the results of this paper were published in a brief form in Letter Ref. 45 . 


\section{BACKGROUND}

In this introductory section, we briefly summarize the known results for higher-dimensional mesoscopic systems (Sec. IIA) and for strongly correlated 1d systems (Sec. IIB). These will serve as a starting point for our work.

\section{A. Mesoscopics of higher-dimensional systems}

During the last three decades, mesoscopic physics of low-dimensional diffusive systems has been attracting a great deal of attention. $46,47,48,49,50,51$ It has been recognized that the effects of the quantum interference and e-e interaction become strongly enhanced in disordered systems of reduced dimensionality $d$, affecting in an essential way the low-temperature transport properties of the systems. These effects lead to a set of remarkable phenomena, including the WL, mesoscopic conductance fluctuations, and the Altshuler-Aronov interaction-induced quantum correction to the conductivity. At still lower temperatures, these effects may drive the system towards strong localization. We now briefly remind the reader the basic facts in this field.

\section{Moderately low temperatures: Weak-localization regime}

The quantum localization is the most prominent manifestation of the quantum interference in disordered systems. At not too low temperatures, the localization effects are cut off by inelastic processes, resulting in a WL correction $^{52} \Delta \sigma_{\mathrm{wl}}$ to the Drude conductivity $\sigma_{\mathrm{D}}$,

$$
\begin{aligned}
\frac{\Delta \sigma_{\mathrm{wl}}}{\sigma_{\mathrm{D}}} & \simeq-\int_{L_{\phi}^{-1}}^{l^{-1}} \frac{(d \mathbf{q})}{\pi \nu_{0} D q^{2}} \\
& \sim-\frac{1}{\nu_{0} D} \begin{cases}\ln \left(\tau_{\phi} / \tau\right), & 2 \mathrm{~d}, \\
\left(D \tau_{\phi}\right)^{1 / 2}, & \text { quasi }-1 \mathrm{~d},\end{cases}
\end{aligned}
$$

where $D$ is the diffusion constant, $\nu_{0}$ the density of states, $l$ the mean free path, $\tau$ the transport mean free time, $\tau_{\phi}$ and $L_{\phi}=\left(D \tau_{\phi}\right)^{1 / 2}$ are the interaction-induced dephasing time and length, respectively. We use notation $(d \mathbf{q})=d^{d} q /(2 \pi)^{d}$. Throughout the paper $\hbar=1$. Another manifestation of the quantum coherence are mesoscopic fluctuations of the conductance and of other observables.

As far as e-e interaction in the WL regime is concerned, it is responsible for two distinctly different main effects. First, it renormalizes elastic scattering on impurities through the creation of virtual electron-hole excitations (screening) with a high energy transfer $\omega$ larger than the temperature $T, T \lesssim|\omega| \lesssim \epsilon_{F}$. Here $\epsilon_{F}$ is the Fermi energy. At sufficiently high $T$ (in the ballistic regime $T \tau \gg 1$ ), i.e., when the relevant spatial scales are smaller than $l$, the screening can be described in terms of a dressing of impurities by Friedel oscillations ${ }^{53,54}$ and yields a
$T$-dependent Drude conductivity 54 At lower $T$ (in the diffusive regime $T \tau \ll 1$ ), effects of this type generate the Altshuler-Aronov correction to the conductivity, ${ }^{46}$

$$
\begin{aligned}
\frac{\Delta \sigma_{\mathrm{AA}}}{\sigma_{\mathrm{D}}} & \simeq-\alpha \int_{L_{T}^{-1}}^{l^{-1}} \frac{(d \mathbf{q})}{\pi \nu_{0} D q^{2}} \\
& \sim \frac{\alpha}{\nu_{0} D} \begin{cases}\ln (T \tau), & 2 \mathrm{~d}, \\
-(D / T)^{1 / 2}, & \text { quasi }-1 \mathrm{~d},\end{cases}
\end{aligned}
$$

where $L_{T} \sim(D / T)^{1 / 2}$ is the thermal length. This correction is perturbative both in the effective interaction constant $\alpha$ and in the disorder strength. The Coulomb interaction corresponds to $\alpha \sim 1$. The $T$ dependence of $\Delta \sigma_{\mathrm{AA}}$ is determined by the thermal smearing of the distribution function, which introduces an infrared cutoff on spatial scales of the order of $L_{T}$. Using the renormalization-group (RG) approach within the replicated $\sigma$-model, Finkel'stein extended this theory to include the interaction nonperturbatively 5

Effects related to the interaction-induced renormalizations and screening are also responsible for a zero-bias anomaly 46,55 in the tunneling density of states as a function of electron energy, $\nu(\epsilon)$. It is worth noting that, in the case of Coulomb interaction, $\nu(\epsilon)$ may become strongly suppressed, $, 55,56,57$

$$
\begin{aligned}
\frac{\nu(\epsilon)}{\nu_{0}} & \sim \exp \left\{-\frac{1}{8 \pi^{2} \nu_{0} D}\left[\ln ^{2}\left(D \kappa^{2} /|\epsilon|\right)\right.\right. \\
& \left.\left.-\ln ^{2}\left(D \kappa^{2} \tau\right)\right]\right\} \lesssim 1, \quad 2 \mathrm{~d},
\end{aligned}
$$

where $\kappa^{-1}$ is the screening radius, while the conductivity can still remain close to its Drude value $\left(\Delta \sigma_{\mathrm{AA}} / \sigma_{\mathrm{D}} \ll 1\right)$.

The second effect of interaction is inelastic e-e scattering which breaks the phase coherence. $\stackrel{46}{ } \mathrm{~A}$ key concept in the localization theory of a disordered Fermi liquid is that of the dephasing rate $\tau_{\phi}^{-1}$. The phase-breaking processes are of crucial importance in the problem of transport since without dephasing the conductivity of low-dimensional systems would be zero at any $T$, see Sec.II A 2 Two qualitatively different sources of dephasing are possible: (i) scattering of electrons by external excitations (in practice, phonons) and (ii) e-e scattering (at low $T$ the dephasing is mostly due to e-e interactions). In either case, at sufficiently high $T$, the dephasing rate $\tau_{\phi}^{-1}$ is high, so that the localization effects are reduced to the WL correction to the Drude conductivity.

A characteristic energy transfer in the real inelastic processes that determine the phase relaxation is restricted by temperature: $|\omega| \lesssim T$, so that the dephasing rate vanishes at zero temperature. The infrared divergency characteristic of a diffusive system makes it necessary to introduce a self-consistent cutoff in the equation for the dephasing rate: ${ }^{46}$

$$
\tau_{\phi}^{-1} \sim \alpha^{2} T \int_{L_{\phi}^{-1}}^{L_{T}^{-1}} \frac{(d \mathbf{q})}{\pi \nu_{0} D q^{2}}
$$




$$
\sim \begin{cases}\frac{\alpha^{2} T}{\nu_{0} D} \ln \left(\frac{\nu_{0} D}{\alpha^{2}}\right), & 2 \mathrm{~d}, \\ \left(\frac{\alpha^{2} T}{\nu_{0} D}\right)^{2 / 3} D^{1 / 3}, & \text { quasi }-1 \mathrm{~d} .\end{cases}
$$

A rigorous calculation necessitates employing the pathintegral technique $\underline{\underline{58}}$

The different energy scales relevant to the disorder renormalization due to virtual processes with $T<|\omega|<$ $\epsilon_{F}$ and to the dephasing due to real inelastic scattering with $|\omega| \lesssim T$ allow for a straightforward separation of these two effects of e-e interaction. At sufficiently high $T$, the conductivity

$$
\sigma(T) \simeq \sigma_{\mathrm{D}}+\Delta \sigma_{\mathrm{wl}}+\Delta \sigma_{\mathrm{AA}}
$$

is close to the Drude value $\sigma_{\mathrm{D}}$. It is worth mentioning that for weak interaction $(\alpha \ll 1)$ the WL correction [Eq. (2.1) with $\tau_{\phi}$ given by Eq. (2.4)] is much stronger than the Altshuler-Aronov correction [Eq. (2.2)]. The WL correction grows with lowering $T$ and eventually becomes strong $\left(\left|\Delta \sigma_{\mathrm{wl}}\right| / \sigma_{\mathrm{D}} \sim 1\right)$ when $L_{\phi}$ reaches $\xi$ or, equivalently, when $\tau_{\phi}^{-1}$ becomes of order $\Delta_{\xi}$. Here $\xi$ is the localization length and $\Delta_{\xi}$ is the corresponding energy level spacing. At this temperature, $\left|\Delta \sigma_{\mathrm{AA}}\right| / \sigma_{\mathrm{D}} \ll 1$ for $\alpha \ll 1$. This means that in a weakly interacting system the strong localization occurs due to the growth of the interference-induced WL correction. In the next subsection, we briefly overview the strong localization regime which emerges at low temperatures, when $\tau_{\phi}^{-1} \lesssim \Delta_{\xi}$.

\section{Low temperatures: Fate of dephasing in the strongly localized regime}

In a pathbreaking paper ${ }^{59}$ Anderson demonstrated that a quantum particle may become localized by a random potential. In particular, in noninteracting systems of one- or two-dimensional geometry even weak disorder localizes all electronic states, $, 2,60$ thus leading to the exactly zero conductivity, $\sigma(T)=0$, whatever temperature $T$. A nonzero $\sigma(T)$ in such systems may only occur due to inelastic scattering processes leading to the dephasing of electrons.

As discussed in Sec. II 1 the WL correction (2.1) to the Drude conductivity of a diffusive system behaves as $\tau_{\phi}^{(2-d) / 2}\left(\ln \tau_{\phi}\right.$ for $\left.d=2\right)$ and diverges with lowering temperature for $d \leq 2$. This singular behavior of the perturbative corrections is a manifestation of strong Anderson localization. It prompts a question as to how the disordered interacting low-dimensional systems conduct at low temperatures, when the localization effects are no longer weak.

It is commonly believed that transport in the localized regime is of the hopping nature. Mott $\underline{61}$ has developed the picture of variable-range-hopping $(\mathrm{VRH})$ as the transport mechanism in the insulating state. The
VRH mechanism of Ref. 61 is well justified in the presence of delocalized phonons which provide inelastic scattering between single-particle states with different energies (phonon-assisted hopping). The finite-temperature conductivity is then proportional to the strength of the electron-phonon coupling and governed by Mott's $\mathrm{VRH}:{ }^{61} \sigma(T) \propto \exp \left[-\left(T_{0} / T\right)^{\mu}\right]$ with $\mu=1 /(d+1)$ depending on the spatial dimensionality $d$. In the presence of a long-range Coulomb interaction, the Coulomb gap in the tunneling density of states modifies 62 the VRH exponent, $\mu=\frac{1}{2}$.

But what if the coupling to phonons is negligibly weak? It was proposed in Ref. 63 that the e-e interaction by itself is sufficient to induce VRH at low $T$. Although the idea ${ }^{63}$ of a phononless VRH was widely exploited in the community, it is, however, in conflict with the argument by Fleishman and Anderson ${ }^{64}$ that elementary hops in the low- $T$ limit are forbidden for a short-range interaction (including the case of $1 / r$ Coulomb interaction in $d<3$ ), because energy conservation cannot be respected when an electron attempts a real transition by exciting an electron-hole pair. Since there is a mismatch in the positions of the energy levels of individual single-particle localized states, inelastic scattering is necessary to broaden these levels to allow for hopping between them. It was argued in Ref. 64 that at low $T$ a short-range e-e interaction alone is not sufficient to broaden the localized states and to induce a nonzero conductivity. The situation is particularly interesting in $1 \mathrm{~d}$ and $2 \mathrm{~d}$, where no mobility edge exists, activation to which otherwise might give $\sigma(T) \neq 0$.

Very recently, the problem was reconsidered in Refs. 65, 66. It was found there that the Anderson localization in Fock space crucially affects the conductivity at low $T$. As a result, the phononless VRH should not exist; instead, the system undergoes a transition at certain temperature $T_{c}$ below which the conductivity vanishes, in agreement with Ref. 64.

The mechanism of low- $T$ transport in low-dimensional disordered systems is thus completely determined by inelastic processes which govern the lifetime of localized states. This lifetime may be viewed as a low-temperature reincarnation of the dephasing time that controls the cutoff of quantum-interference effects at higher $T$. Therefore, an analysis of the WL effects turns out to be important also for understanding of the establishment of the strong localization with lowering $T$.

\section{B. Strongly correlated $1 \mathrm{~d}$ electron systems}

This paper is concerned with transport in a singlechannel quantum wire, where both disorder and interaction more strongly modify the transport properties as compared to higher dimensions. In the absence of disorder, the e-e correlations drive the system into the nonFermi liquid state known as Luttinger liquid. Yet another peculiarity of the single-channel $1 \mathrm{~d}$ system is that the bal- 
listic motion on short scales crosses over in the absence of interaction directly to the localization regime, with no diffusive dynamics on intermediate scales. We are now going to expand on these known features of $1 d$ electronic systems.

\section{Noninteracting case: Anderson Localization}

We first briefly recall the peculiarities of $1 \mathrm{~d}$ noninteracting disordered systems. In the absence of interaction, transport in a disordered single-channel 1d gas has been studied in great detail $67,68,69,70,71,72,73,74,75$ It is known that arbitrarily weak disorder leads to the localization of all electronic states in this system, with the localization length $\xi$ being the mean free path. The dc conductivity is then zero, $\sigma(T) \equiv 0$ for any $T$, since the temperature merely determines the distribution function over the localized states.

The characteristic spatial and temporal scales of the problem are probed in the absence of interactions by the ac response at external frequency $\Omega$. The appropriate technique for the calculation of the response, which exploits the separation of fast and slow degrees of freedom in the case of weak disorder, $\epsilon_{F} \tau \gg 1$, was put forward in a pioneering paper by Berezinskii; 69 later, alternative approaches were developed by Abrikosov and Ryzhkin ${ }^{71}$ and by Berezinskii and Gor'kov ${ }^{73}$ In the limit of low frequency, $\Omega \tau \ll 1$, the leading contribution to the ac conductivity is given by the Berezinskii-Mott formula: ${ }^{69,70}$

$$
\operatorname{Re} \sigma(\Omega) / \sigma_{\mathrm{D}}=4(\Omega \tau)^{2} \ln ^{2}(\Omega \tau) .
$$

In the opposite (ballistic) limit of high frequency, $\Omega \tau \gg$ 1 , the conductivity $\sigma(\Omega)$ is approximately equal to the Drude formula, $\sigma_{\mathrm{D}}(\Omega)=\sigma_{\mathrm{D}} /(1-i \Omega \tau)$, with a quantum interference (WL) correction arising at fourth order in the expansion in $(\Omega \tau)^{-1} \ll 1$. At $\Omega \tau \sim 1$, the ballistic regime crosses over directly to the strongly localized regime, Eq. (2.6), so that the diffusive regime is absent in the single-channel quantum wire without e-e interaction.

\section{Clean quantum wires with interaction: Luttinger liquid}

The interest in the role of e-e interaction in quantum wires is largely inspired by the fact that in $1 \mathrm{~d}$ the interaction breaks down the Landau's Fermi liquid approach. Owing to the particular geometry of the Fermi surface, systems of dimensionality one are unique in that the e-e correlations change the noninteracting picture completely and lead to the formation of a strongly correlated state. A remarkable example of the correlated 1d electron phase is the Luttinger-liquid model (for a review see, e.g., Refs. 27, 28,29,30,31,32). The Luttinger-liquid correlations show up in a power-law singularity of the tunneling density of states at low energy $\epsilon$ counted from the Fermi surface,

$$
\nu(\epsilon) \sim \nu_{0}(|\epsilon| / \Lambda)^{\beta} .
$$

Here $\nu_{0}$ is the density of states in the absence of interaction, $\Lambda$ is the ultraviolet cutoff (determined by the band width, the Fermi energy, or the range of interaction). The exponent $\beta$ is determined by the interaction strength and depends on the system geometry (tunneling into the bulk of a wire differs from tunneling into the end of a wire). The suppression of the tunneling density of states in Luttinger liquid is similar to the interaction-induced zero-bias anomaly in higher dimensions, Eq. (2.3).

According to a common wisdom, electronic excitations do not exist in Luttinger liquid and a proper language to describe the strongly correlated electrons is the language of bosonic excitations. This notion is based on the fact that the bosonic density-fields in a clean Luttinger liquid do not decay in the linearized-spectrum approximation, when a finite curvature of the electron spectrum is neglected.

\section{Renormalization of an impurity in Luttinger liquid}

Interactions between oppositely moving electrons generate charge- and spin-density wave correlations that lead to striking transport properties of Luttinger liquid in the presence of impurities. The densitywave correlations yield a strong renormalization of an impurity: $76,77,78,79,80,81$ if the bare strength of the impurity is small, the renormalized backscattering amplitude $U\left(2 k_{F}, \epsilon\right)$ seen by an electron with energy $\epsilon$ scales as

$$
U\left(2 k_{F}, \epsilon\right) \propto(\Lambda /|\epsilon|)^{a}
$$

where $a$ is determined by the interaction strength. As a result, a single impurity in the infinite Luttinger liquid with a repulsive interaction effectively decouples the wire into two independent pieces, thus leading to a vanishing linear conductance at zero temperature. This conclusion remains valid no matter how weak the interaction is.

\section{Many impurities: Disordered Luttinger liquid}

The conductivity of a disordered Luttinger liquid was discussed for the first time more than 30 years ago in Refs. 76.77.78 in terms of the renormalization of individual impurities (see also Refs. 82,83 for early attempts to construct the phase diagram of a disordered Luttinger liquid). In the beginning of the 80's, Apel and Rice 33 combined the scaling arguments developed for noninteracting systems $60,72,84$ with the results of the theory of a clean Luttinger liquid. Later, the problem of transport in a disordered Luttinger liquid was studied by perturbative in disorder methods based on bosonic field theories in Refs. 34,85,86. A small correction to the quantized conductance of a short quantum wire due to a Gaussian disorder was derived in Ref. 86 (for a multichannel wire see Ref. 87). A detailed exposition of these issues can be found in the recent lecture course Ref. 88 . 
The RG equations for a disordered Luttinger liquid were derived by Giamarchi and Schulz $\stackrel{34}{*}^{\text {Their approach }}$ bears a similarity with the Finkel'stein RG ${ }^{55}$ developed earlier for higher-dimensional diffusive systems (both RG schemes treat disorder perturbatively and e-e interaction exactly). The flow equations of Ref. 34 describe, in addition to the renormalization of impurities by interaction (Sec. IIB 3), also a renormalization of the interaction by disorder. A sufficiently strong attractive interaction was shown to yield a metal-insulator transition ${ }^{34}$ at zero $T$ with changing interaction strength. A repulsive interaction was predicted to enhance the disorder-induced localization. In the latter case, the RG equations show that at a certain temperature (such that $L_{T} \sim \xi$ ) the renormalized disorder becomes strong. This means that the disordered interacting 1d system enters a "strongcoupling regime", where the Drude-like approach breaks down. At higher temperatures, these equations describe the temperature dependence of an effective scattering time renormalized by interaction (which can be viewed as screening of impurities by Friedel oscillations), thus yielding a temperature-dependent Drude conductivity,

$$
\sigma_{\mathrm{D}}(T) \propto(T / \Lambda)^{2 a} .
$$

Recently, a crossover between Eqs. (2.9) and (2.2) with increasing number of channels was studied in Ref. 43 .

It is important to emphasize that the RG approach formalizes the renormalization of the Drude conductivity due to the $T$-dependent screening but does not capture the essential physics of the Anderson localization. In particular, the RG equations of Ref. 34 miss the interference effects (coherent scattering on several impurities) that lead to the WL correction to the conductivity. As we demonstrate below, it is the WL correction that governs the temperature dependence of the conductivity at moderately high temperatures for the case of weak interaction and eventually drives the quantum wire into the strong-localization regime with decreasing temperature. Another related deficiency of the RG scheme of Ref. 34 is the treatment of the scalings with length and $u / T$ as interchangeable. While this approach is justified for the "elastic renormalization" of disorder (i.e., the screening effects), it does not properly account for the dephasing (since the dephasing length $L_{\phi}$ is in general different from $L_{T}$, see Sec. III B 1 and Sec. IV C).

\section{Dephasing and localization in disordered Luttinger liquid}

As already mentioned in Sec. IIB 4, a key ingredient of transport theory as regards the WL and the interactioninduced dephasing in a strongly correlated $1 \mathrm{~d}$ system is missing. Some papers (e.g., Refs. 34,81) have suggested that the dephasing length that controls localization effects in a disordered Luttinger liquid is $L_{T}=u / T$, where $u$ is the plasmon velocity. According to this approach, the interference effects get strong with lowering $T$ at $L_{T} \sim \xi$.
An alternative approach ${ }^{33}$ is predicated on the assumption that the dephasing rate is determined by the singleparticle properties of a clean Luttinger liquid. On top of that, one might think that since in the case of linear dispersion the clean interacting electron system can be equivalently represented in terms of noninteracting bosons, the interaction should not induce any dephasing at all. The conductivity would then be exactly zero at any $T$. As we demonstrate below, none of the approaches captures the essential physics of dephasing in the conductivity of a disordered $1 \mathrm{~d}$ system.

As for the strongly localized regime, recently Ref. 37 used bosonization to study the problem and concluded that transport is of variable-range-hopping character. Subsequently, Ref. 38 argued that this result requires a nonzero coupling to a thermal bath, in agreement with Ref. 64, see Sec. II A2,

\section{Summary of known results: 1d vs 2d}

We summarize the basic facts discussed in Secs. IA and 【B in Table If for simplicity, we compare here $2 \mathrm{~d}$ and single-channel $1 \mathrm{~d}$ systems. It turns out that the difference between the disordered Luttinger liquid and the higher-dimensional (quasi-1d and 2d) systems is not so strong as one might think judging by the proverbial nonFermi-liquid character of the Luttinger liquid.

\section{DISORDERED LUTTINGER LIQUID}

\section{A. Formulation of the problem}

Let us now introduce the model we are going to study. We consider a single-channel infinite wire at sufficiently low temperatures, much lower than the bandwidth and the Fermi energy. The effective low-energy theory can then be described by the Luttinger model, $27,28,29,30,31,32$ with a linear dispersion of the electronic spectrum $\epsilon_{k}=$ $k v_{F}$, where $v_{F}$ is the Fermi velocity. We use the notion of right- and left-movers corresponding to two Fermi points $k \sim \pm k_{F}$. The fermionic operators $\psi_{\mu}$ are then labeled by $\mu= \pm$ for the right-/left-movers.

We consider a finite-range (screened) pairwise e-e interaction potential $V\left(x-x^{\prime}\right)$. We need only its Fourier transforms at $q=0$ and $q= \pm 2 k_{F}: V_{\mathrm{f}}$ and $V_{\mathrm{b}}$, respectively, which give the amplitudes of forward and backward scattering. In terms of the $g$-ology, $\stackrel{27,28,32}{2}$ the interaction in a homogeneous system is characterized by three coupling constants $g_{1}=V_{\mathrm{b}}$ (e-e backscattering), $g_{2}=V_{\mathrm{f}}$ (forward scattering between right- and left-movers), and $g_{4}=V_{\mathrm{f}}$ (forward scattering of right-/left-movers on right-/leftmovers). In several places below, it will be convenient to treat $g_{2}$ and $g_{4}$ as independent parameters.

Next, we introduce weak $\left(\epsilon_{F} \tau_{0} \gg 1\right)$ white-noise dis- 
TABLE I: The $2 \mathrm{~d}-1 \mathrm{~d}$ vocabulary.

\begin{tabular}{|c|c|}
\hline $2 \mathrm{~d}$ & $1 d$ \\
\hline $\begin{array}{l}\text { disorder, no interaction: } \\
\qquad \sigma(T)=0\end{array}$ & $\begin{array}{c}\text { disorder, no interaction: } \\
\qquad \sigma(T)=0\end{array}$ \\
\hline $\begin{array}{l}\text { interaction, common tool: } \\
\text { fermionic diagrams }\end{array}$ & $\begin{array}{l}\text { interaction, common tool: } \\
\text { bosonization }\end{array}$ \\
\hline field theory: $\sigma$-model & field theory: \\
\hline$Q$-matrices & bosonic density fields \\
\hline $\begin{array}{l}\text { zero-bias anomaly in } \\
\text { tunneling DoS, Eq. (2.3) }\end{array}$ & $\begin{array}{l}\text { Luttinger ("non-Fermi") liquid } \\
\text { power-law DoS, Eq. (2.7) }\end{array}$ \\
\hline $\begin{array}{l}\text { T-dependent screening } \\
\text { (Friedel oscillations) }\end{array}$ & $\begin{array}{l}\text { renormalization of impurity } \\
\text { (Friedel oscillations), Eq. (2.8) }\end{array}$ \\
\hline $\begin{array}{l}\text { Altshuler-Aronov correction } \\
\text { to the conductivity, Eq. (2.2) }\end{array}$ & $\begin{array}{c}\text { renormalization of disorder } \\
\longrightarrow \sigma_{\mathrm{D}}(T), \text { Eq. }\end{array}$ \\
\hline $\begin{array}{c}\text { Finkel'stein RG: } \\
\text { disorder (perturbative) } \\
\text { \& interaction (exact) }\end{array}$ & $\begin{array}{c}\text { Giamarchi-Schulz RG: } \\
\text { disorder (perturbative) } \\
\& \text { interaction (exact) }\end{array}$ \\
\hline$L_{T} \sim \xi:$ strong & $L_{T} \sim \xi$ : strong coupling \\
\hline$\neq$ Anderson localization & $\neq$ Anderson localization \\
\hline $\begin{array}{l}\quad L_{\phi} \sim \xi, \quad \Delta \sigma_{\mathrm{wl}} \sim \sigma_{\mathrm{D}}: \\
\text { strong Anderson localization }\end{array}$ & $\begin{array}{c}\quad L_{\phi} \sim \xi, \quad \Delta \sigma_{\mathrm{wl}} \sim \sigma_{\mathrm{D}}: \\
\text { strong Anderson localization }\end{array}$ \\
\hline $\begin{array}{l}\text { weak localization correction } \\
\text { to the conductivity, Eq. 2.1 }\end{array}$ & $\begin{array}{l}\text { this work, } \\
\text { Secs. VIVII }\end{array}$ \\
\hline dephasing time, Eq. (2.4) & this work, Secs. VIVII \\
\hline
\end{tabular}

order, described by the correlation function

$$
\left\langle U(x) U\left(x^{\prime}\right)\right\rangle=\delta\left(x-x^{\prime}\right) / 2 \pi \nu_{0} \tau_{0},
$$

where $\tau_{0}$ is the elastic scattering time and $\nu_{0}=1 / \pi v_{F}$ is the density of states per spin in the absence of interaction.

The Hamiltonian for spinful electrons takes the form:

$$
\begin{aligned}
H & =H_{\mathrm{kin}}+H_{\mathrm{ee}}+H_{\mathrm{dis}} \\
H_{\mathrm{kin}} & =\sum_{k, \mu= \pm, \sigma} v_{F}\left(\mu k-k_{F}\right) \psi_{\mu \sigma}^{\dagger}(k) \psi_{\mu \sigma}(k) \\
H_{\mathrm{ee}} & =\frac{1}{2} \sum_{\mu, \sigma, \sigma^{\prime}} \int d x\left\{n_{\mu, \sigma} g_{2} n_{-\mu, \sigma^{\prime}}+n_{\mu, \sigma} g_{4} n_{\mu, \sigma^{\prime}}\right.
\end{aligned}
$$

In what follows we omit the tilde over the shifted coupling $g_{2}$. The second simplification is related to the $g_{4^{-}}$ term in Eq. (3.10). Naively, one could think it is simply 
zero, since it describes a local interaction between identical fermions. In fact, the situation is slightly more tricky. While yielding no genuine interaction, this term generates a shift of the velocity due to a quantum anomaly, $v_{F} \rightarrow v_{F}^{*}$, where

$$
v_{F}^{*}=v_{F}+\frac{g_{4}}{2 \pi} .
$$

This is also straightforwardly seen in the bosonization approach, see Sec. IV]

Therefore, the complete set of parameters defining the spinless problem includes $v_{F}^{*}, g_{2}, \tau_{0}$, and $\Lambda$, with the interaction characterized by a single coupling $g_{2}$. It is customary to define the corresponding dimensionless parameter in the form

$$
K=\left(\frac{1-g_{2} / 2 \pi v_{F}^{*}}{1+g_{2} / 2 \pi v_{F}^{*}}\right)^{1 / 2} .
$$

Note that in a $1 \mathrm{~d}$ system with $g_{2}=g_{4}$ Eq. (3.13) is identical to another conventional representation of $K$ in terms of the bare velocity $v_{F}$,

$$
K=\frac{1}{\left(1+g_{2} / \pi v_{F}\right)^{1 / 2}} .
$$

For the case of a weak interaction (considered in the present paper), $K \simeq 1-\alpha$, where

$$
\alpha=\frac{g_{2}}{2 \pi v_{F}^{*}} \simeq \frac{g_{2}}{2 \pi v_{F}} \ll 1
$$

is the dimensionless strength of the interaction.

\section{B. Qualitative discussion: Luttinger-liquid renormalization vs Fermi-liquid dephasing}

Having formulated the model in Sec. IIA, we turn now to a discussion of our strategy in its analysis. In principle, one could proceed in a number of alternative ways:

(i) First of all, one can bosonize the action and try to study the transport properties of interacting electrons in the bosonic language, Sec. IV] While this approach is very powerful for the problem of $1 \mathrm{~d}$ interacting fermions, it turns out to be poorly suited for the analysis of transport problems in the presence of disorder. For this reason, we develop an alternative "two-step procedure".

(ii) The two-step procedure combines the language of bosonization for high energies with a fermionic treatment of processes with low-energy transfers. This method is discussed on the qualitative level in the remainder of this subsection and is implemented in Secs. IVIVI below.

(iii) Finally, one more alternative is the "functional bosonization" approach $\underline{89,90,91,92,93}$ This method also combines the languages of fermionic (electrons) and bosonic (plasmons) excitations, but in contrast to the two-step procedure this is done in a uniform fashion, so that the processes with high and low energy transfers are treated on the same footing. This method yields results identical to the two-step approach and is presented in Sec. VII]

Let us describe now the ideas underlying our two-step approach. The method is based on the separation of two types of processes: (i) "elastic renormalization" of disorder, which is associated with virtual transitions with energy transfers $|\omega| \gtrsim T$ and (ii) inelastic processes with $|\omega| \lesssim T$ which lead to dephasing in quantum-interference effects and thus to a destruction of the Anderson localization. This separation of energy scales is very much similar to that in physics of mesoscopic phenomena in higher-dimensional case, see Sec. 【A.

\section{Step 1: Luttinger-liquid renormalization}

We begin by considering the Drude conductivity under the condition that the dephasing time $\tau_{\phi}$ is much shorter than the transport time of elastic scattering off disorder $\tau$. The source of the strong dephasing may be external (say, interaction with phonons, or Coulomb interaction with "environment") or intrinsic, that is the inelastic e-e interaction within the quantum wire. In the latter case, the limit $\tau_{\phi} \ll \tau$ is achieved at sufficiently high $T$, as shown below in Sec. VI For $\tau_{\phi} \ll \tau$, the Andersonlocalization effects are strongly suppressed by dephasing.

To leading order in $\tau_{\phi} / \tau \ll 1$, the conductivity is given by the Drude formula

$$
\sigma_{\mathrm{D}}=e^{2} \rho v_{F}^{2} \tau,
$$

where $\rho$ is the compressibility. The Drude conductivity (3.16) depends on $T$ through a $T$-dependent renormalization of the static disorder: $34,77,78,79,80$

$$
\tau(T)=\tau_{0}(T / \Lambda)^{2 a} .
$$

For spinless electrons, the exponent in Eq. (3.17) reads

$$
a=1-K \simeq \alpha .
$$

In principle, the interplay of disorder and interaction leads to the renormalization of both disorder, Eq. (3.17), and interaction $\stackrel{34}{=}$ However, the value of $\alpha$ in Eq. (3.18) is given by the bare interaction constant (the one in a clean system), see Sec. IV for details.

The Luttinger-liquid renormalization (3.17) is similar to the Altshuler-Aronov corrections $\underline{46}$ [Eq. (2.2)] in higher dimensionalities. The underlying physics of the elastic renormalization of disorder can be described in terms of the $T$-dependent screening of individual impurities and scattering by slowly decaying in real space Friedel oscillations, similarly to higher-dimensional systems $\frac{53.54}{4}$ At this level, the only peculiarity of Luttinger liquid as compared to higher dimensionalities is that the renormalization of $\tau$ is more singular and necessitates going beyond the Hartree-Fock (HF) approach even for the weak interaction. 94 
Technically, Eq. (3.17) can be derived by a variety of methods: either fermionic 27,79 or bosonic ${ }^{34,80}$ In particular, a conceptually important framework is based on the bosonic RG procedure developed by Giamarchi and Schulz $\stackrel{34}{=}$ This approach allows one to integrate out all energy scales between $\Lambda$ and $T$, which are responsible for the elastic Luttinger-liquid renormalization due to virtual e-e scattering processes. We will discuss the features of the bosonic RG approach ${ }^{34}$ relevant to our analysis in more detail in Sec. IV

The renormalization of $\tau$ stops with decreasing $T$ at $T \tau(T) \sim 1$. Physically, this is due to the fact that the long-range Friedel oscillations are cut off even at zero $T$ on the spatial scale of the order of the disorder-induced mean free path. The condition

$$
T \tau(T) \sim 1
$$

gives the zero- $T$ localization length

$$
\xi \propto \tau_{0}^{1 /(1+2 a)} .
$$

It is important to stress, however, that Eq. (3.19) does not correctly predict the onset of localization, in contrast to the argument made in Refs. 34,81. This can be seen, in particular, by noting that the temperature $T \sim \tau^{-1}$ does not depend on the strength of interaction for small $\alpha$, whereas it is evident that for noninteracting electrons there is no interaction-induced dephasing (the dephasing length is infinite, $L_{\phi}=\infty$ ) and hence $\sigma(T)=0$ for any $T$ due to the Anderson localization, see Sec. IIB 1. The onset of strong localization occurs at $L_{\phi} \sim \xi$, as in the case of higher dimensionality, see the discussion in Sec. IIA The temperature $T_{1}$ below which the Anderson-localization effects become strong is determined in $1 \mathrm{~d}$ by the condition

$$
\tau\left(T_{1}\right) \sim \tau_{\phi}\left(T_{1}\right) .
$$

At this temperature, the quantum-interference effects leading to the WL correction $\Delta \sigma_{\mathrm{wl}}$ to the conductivity are no longer weak,

$$
\left|\Delta \sigma_{\mathrm{wl}}\left(T_{1}\right)\right| \sim \sigma_{\mathrm{D}}\left(T_{1}\right) .
$$

\section{Step 2: Fermi-liquid dephasing}

It is worth stressing that the Luttinger-liquid physics in the present problem is fully accounted for by Eq. (3.17), i.e., all the Luttinger-liquid power-law singularities are now incorporated in the renormalization of $\tau(T)$. At this point, we are left with the system of interacting electrons with a new effective "Fermi energy" $T$. These electrons are scattered by disorder with renormalized $\tau_{0} \rightarrow \tau(T)$, which is still weak for $T \tau(T) \gg 1$.

What remains after the renormalization is the e-e scattering with energy transfers $|\omega| \lesssim T$. The interaction is thus still important and leads to real inelastic processes.
The physics of these processes is in essence equivalent to the Fermi-liquid physics of dephasing in higher dimensionalities. Having integrated out the virtual transition, we have to choose a controllable method of dealing with the dephasing. The bosonization approach (whose advantage is that it can be straightforwardly formulated for the arbitrary strength of interaction) is not particularly beneficial here. Although, in principle, it contains all effects of the Anderson localization in 1d, the machinery of bosonization is poorly suited to deal with such ingredients of the localization theory as the interference and dephasing. Even for noninteracting electrons, it is by far not straightforward to derive the Anderson localization [for instance, to obtain the Berezinskii-Mott formula (2.6) for the case of weak disorder] in the language of bosonization. For this reason, we refermionize the theory at this step, Secs. VIVI

\section{BOSONIZATION APPROACH}

\section{A. Bosonized action}

As discussed in Sec. IIIB the first part of our program, namely the renormalization of the model due to highenergy processes, is done most efficiently in the framework of the bosonization technique. In this section, we present a calculation of $\tau(T)$, using the RG scheme developed by Giamarchi and Schulz $\stackrel{34}{=}$ After a brief description of the derivation of the RG equations, we will discuss an important subtlety of the scheme, $\stackrel{34}{=}$ namely a mixing of disorder and interaction.

For simplicity, we consider here the spinless case (in notation of Ref. 34, we set $g_{1 \perp}=g_{2 \perp}=0$ and hence $\left.u_{\rho}=u_{\sigma}, K_{\rho}=K_{\sigma}\right)$. As discussed in Sec. IIIA for spinless electrons the backscattering amplitude $g_{1}$ can be absorbed into $g_{2}$, and $g_{4}$ into $v_{F}^{*}$, so that only one interaction coupling constant remains $\left(g_{2}\right)$, yielding the dimensionless Luttinger-liquid parameter $K$, Eq. (3.13).

Using the boson representation of the fermionic operators,$\stackrel{32}{=}$ the Hamiltonian can be expressed in terms of the boson operators $\phi$ and $\Pi$ that satisfy the commutation relation $\left[\Pi(x), \phi\left(x^{\prime}\right)\right]=-i \delta\left(x-x^{\prime}\right)$. In a given realization of disorder the Hamiltonian reads

$$
\begin{aligned}
H & =\frac{1}{2 \pi} \int d x\left\{u K(\pi \Pi)^{2}+\frac{u}{K}\left(\partial_{x} \phi\right)^{2}\right\} \\
& +\frac{1}{2 \pi \lambda} \int d x\left\{\mathcal{U}_{b}^{*}(x) \exp (2 i \phi)+\text { H.c. }\right\}
\end{aligned}
$$

with

$$
u=\left[\left(v_{F}^{*}\right)^{2}-g_{2}^{2} / 4 \pi^{2}\right]^{1 / 2} .
$$

The ultraviolet length scale $\lambda$ is related to the energy cutoff $\Lambda$ as

$$
\lambda=u / \pi \Lambda .
$$


We will concentrate on the case of e-e repulsion, implying that $K<1$. The disorder-induced forward scattering amplitude $\mathcal{U}_{f}(x)$ is absorbed ${ }^{34}$ in the quadratic part of $H$ as a shift of $\phi(x)$.

In order to perform the disorder averaging, one introduces replicas, $\phi_{n}$. In the resulting action the interaction (Luttinger-liquid) term $S_{L L}$ is quadratic in fields $\phi_{n}$, while disorder generates a term $S_{D}$ of $\cos 2 \phi$-type which breaks the Gaussian character of the theory:

$$
\begin{aligned}
S & =\sum_{n} S_{L L}\left[\phi_{n}\right]+\sum_{n, m} S_{D}\left[\phi_{n}, \phi_{m}\right] \\
S_{L L} & =\frac{1}{u K} \int \frac{d x d \tau}{2 \pi}\left\{\left[\partial_{\tau} \phi_{n}(x, \tau)\right]^{2}+u^{2}\left[\partial_{x} \phi_{n}(x, \tau)\right]^{2}\right\} \\
S_{D} & =-D_{b} \int \frac{d x d \tau d \tau^{\prime}}{(2 \pi \lambda)^{2}} \cos \left\{2\left[\phi_{n}(x, \tau)-\phi_{m}\left(x, \tau^{\prime}\right)\right]\right\}
\end{aligned}
$$

where $D_{b}=v_{F}^{*} / 2 \tau_{0}$. The integration over the imaginary times is performed within the interval $0 \leq \tau \leq 1 / T$.

The conductivity is expressed in terms of the currentcurrent correlation function at zero momentum via the Kubo formula:

$$
\sigma(\Omega)=-\frac{e^{2}}{i \Omega}\left[\frac{v_{F}}{\pi}-\int_{0}^{1 / T} d \tau e^{i \Omega_{n} \tau} \mathcal{K}(\tau)\right]_{i \Omega_{n} \rightarrow \Omega+i 0}
$$

with

$$
\mathcal{K}(\tau)=\int d x\left\langle T_{\tau} j(x, \tau) j(0,0)\right\rangle,
$$

where in the bosonic language the currents are expressed via the field $\phi(x, \tau)$ as

$$
j(x, \tau)=\frac{i}{\pi} \partial_{\tau} \phi(x, \tau) .
$$

The first (diamagnetic) term in Eq. (4.7) is canceled by a contribution of the singularity at $\tau=0$ in the second term. It is worth noting that, strictly speaking, the correlation function $\left\langle T_{\tau} \partial_{\tau} \phi(x, \tau) \partial_{\tau} \phi(0,0)\right\rangle$ is a reducible (with respect to the interaction) correlator, when it is calculated with the use of $S_{L L}$ as given by Eq. (4.5). On the contrary, the conductivity is given by the irreducible current-current correlator. However, at zero external momentum $Q$, the two correlators are equal to each other, since the polarization operator vanishes at $Q=0$ for arbitrary nonzero frequency. This should be contrasted with a calculation of the conductance of a finite interacting wire: the latter is related to the response at finite $Q$ and the Kubo formula should be supplemented with an analysis of the effect of renormalization of the electric field by e-e interaction. ${ }^{95,96}$

Note that the authors of Ref. 34 did not calculate the conductivity using the Kubo formula (4.7), (4.8), (4.9). Instead, they found the renormalized value of $1 / \tau$ and substituted it into the Drude formula, Eq. (3.16) (which can be represented as a certain approximation in the framework of the memory-function formalism, see, e.g., Refs. 78, 97). In view of technical difficulties, in this paper we do not attempt to evaluate the conductivity, including the localization effects, by using the Kubo formula in the bosonic language either. Having used the bosonization for the analysis of the high-energy renormalizations, we refermionize the theory and calculate the conductivity in the fermionic language, Sec. VI]

\section{B. Giamarchi-Schulz RG}

Giamarchi and Schulz ${ }^{34}$ performed the renormalization of the coupling constants $K, u$, and $D_{b}$ in the action (4.4) upon rescaling the ultraviolet cutoff $\lambda \rightarrow L=\lambda \exp (\ell)$. Their analysis is done to first order in the dimensionless strength of disorder

$$
\mathcal{D}=2 D_{b} \lambda / \pi u^{2} .
$$

To this order, one can omit replica indices in Eq. (4.4). Before presenting the RG equations, we discuss a subtle point of the approach developed in Ref. 34. Specifically, the coupling constant $\tilde{K}$ in terms of which the RG equations are obtained in a natural way, is not simply $K$ but rather contains an admixture of disorder. We now briefly reproduce the corresponding argument from Ref. 34 . In the fermionic representation, the part of the action related to the disorder-induced backscattering acquires, after the disorder averaging, the form

$$
\begin{aligned}
S_{D} & =-D_{b} \int d x d \tau d \tau^{\prime} \psi_{+}^{\dagger}(x, \tau) \psi_{-}(x, \tau) \\
& \times \psi_{-}^{\dagger}\left(x, \tau^{\prime}\right) \psi_{+}\left(x, \tau^{\prime}\right) .
\end{aligned}
$$

In order to derive the RG equations, Giamarchi and Schulz singled out the ultraviolet contribution of close times $u\left|\tau-\tau^{\prime}\right| \leq \lambda$, splitting the time integration into two parts:

$$
\begin{aligned}
S_{D} & =-D_{b} \int_{u\left|\tau-\tau^{\prime}\right|>\lambda} d x d \tau d \tau^{\prime} \\
& \times \psi_{+}^{\dagger}(x, \tau) \psi_{-}(x, \tau) \psi_{-}^{\dagger}\left(x, \tau^{\prime}\right) \psi_{+}\left(x, \tau^{\prime}\right) \\
& -\frac{2 D_{b} \lambda}{u} \int d x d \tau \\
& \times \psi_{+}^{\dagger}(x, \tau) \psi_{-}(x, \tau) \psi_{-}^{\dagger}(x, \tau) \psi_{+}(x, \tau) .
\end{aligned}
$$

The second term in Eq. (4.13) is local in time and thus equivalent to an interaction term generated by $g_{1^{-}}$ processes. For this reason, the authors of Ref. 34 redefined the interaction constant, $\tilde{g_{1}} \equiv g_{1}-2 D_{b} \lambda / u$. For the case of spinless electrons considered here, this is equivalent to a modification of the constant $g_{2}$ :

$$
\tilde{g_{2}}=g_{2}+2 D_{b} \lambda / u
$$


In terms of the modified interaction constants the RG equations 34 (in the spinless case only three equations out of original six remain) are:

$$
\begin{aligned}
d \tilde{K} / d \ell & =-\tilde{K}^{2} \mathcal{D} / 2, \\
d \tilde{u} / d \ell & =-\tilde{u} \tilde{K} \mathcal{D} / 2, \\
d \mathcal{D} / d \ell & =(3-2 \tilde{K}) \mathcal{D},
\end{aligned}
$$

where $\tilde{u}$ and $\tilde{K}$ are related to the modified interaction constant

$$
\tilde{g_{2}}(\ell)=g_{2}(\ell)+\pi u \mathcal{D}(\ell)
$$

according to Eqs. (4.2) and (3.13), respectively.

The modification of the plasmon velocity $u$ by disorder, as well as its renormalization, can be neglected in Eq. (4.18), since this generates higher powers of $\mathcal{D}$. Then Eq. (4.16), which describes the renormalization of $u$, turns out to have no influence on the renormalization of disorder and interaction, described by the remaining two equations. [In fact, Eqs. (4.15), (4.16) show that $\tilde{K} / \tilde{u}$ is the invariant of the flow.] Therefore, we will concentrate on the coupled set of the flow equations (4.15), (4.17). It follows from Eq. (4.17) that for $\tilde{K}<3 / 2$ the strength of disorder $\mathcal{D}(\ell)$ increases upon renormalization. At the same time, the value of $\tilde{K}$ decreases. The system flows then to the strong-coupling regime where $\mathcal{D}$ becomes of order one and the first-order treatment of disorder is no longer valid.

The decrease of $\tilde{K}(\ell)$ upon renormalization suggests that the initially weak interaction ( $K$ close to 1 ) becomes strong upon approaching the strong coupling regime. However, this is not so, as we demonstrate below: the decrease of $\tilde{K}$ occurs solely due to the admixture of increasing disorder in this quantity.

It is also worth noticing that Eq. (4.15) seems to generate finite interaction due to the scattering off disorder in the noninteracting case. However, as verified in Ref. 34, this is not the case. The point is that the condition $\tilde{K}=1$ does not mean that the system is noninteracting, because of the mixing of interaction and disorder in Eq. (4.18). Therefore, it is desirable to rewrite Eqs. (4.15), (4.17) in terms of the true interaction constant $K$ instead of the one modified by the admixture of disorder, $\tilde{K}$ (see Fig. 9.6 in Ref. 32).

Let us first consider the case of weak interaction, 1 $K \simeq \alpha \ll 1$, retaining only the terms of first order in $\alpha$ in the RG equations. From Eqs. (3.13), (4.2), (4.10), and (4.14) we get, to first order in $\alpha$,

$$
\tilde{K}=1-\alpha-\mathcal{D} / 2+\alpha \mathcal{D} / 2 .
$$

Substituting this formula in Eq. (4.17), we express the flow equation for the disorder strength in terms of the renormalized true interaction constant $\alpha(\ell)$ :

$$
d \mathcal{D} / d \ell=\mathcal{D}+2 \alpha \mathcal{D}+\mathcal{D}^{2} .
$$

In this equation, the first term in the right-hand side describes Ohm's law, while the second term $(\propto \alpha)$ yields the Luttinger-liquid renormalization of $\tau$, Eq. (3.17).

The last (quadratic-in-disorder) term in Eq. (4.20) is beyond the accuracy of the derivation of the $\mathrm{RG}$ equations and should be neglected. We note, however, that this spurious term is hidden in the RG equations of Ref. 34. Accidentally, this spurious quadratic term qualitatively mimics the effect typical for the WL. Clearly, this purely accidental similarity does not hold on the quantitative level: the true $\mathrm{WL}$ - when included into consideration - would only appear in the r.h.s. of Eq. (4.20) at $\mathcal{D}^{3}$ order.

Now let us turn to the flow equation (4.15) for the interaction strength. We rewrite it using Eqs. (4.19) and (4.20) in the form

$$
d \alpha / d \ell=-3 \alpha \mathcal{D} / 2-\mathcal{D}^{2} .
$$

Again, the last (quadratic-in-disorder) term here is beyond the accuracy of the calculation and has to be neglected (this unphysical term generates interaction from disorder). It is important that the linear-in-disorder term in Eq. (4.21) vanishes for $\alpha=0$. In other words, disorder does not generate interaction, which was not trivial to see from Eq. (4.15).

To summarize, for a weak interaction, $\alpha \ll 1$, the set of two scaling equations for $\alpha$ and $\mathcal{D}$ reads

$$
\begin{aligned}
d \alpha / d \ell & =-3 \alpha \mathcal{D} / 2, \\
d \mathcal{D} / d \ell & =(1+2 \alpha) \mathcal{D} .
\end{aligned}
$$

One sees that Eq. (4.22) yields decreasing $\alpha(\ell)$ : while the disorder strength grows with increasing $L$, the interaction gets weaker ("disorder kills interaction" — Ref. 32; also Ref. 98), in contrast to a naive expectation based on Eq. (4.15). This resembles the behavior of the renormalized interaction in $2 \mathrm{~d}$ diffusive systems. Specifically, according to the Finkel'stein RG equations,, 55 the combination of the singlet and triplet amplitudes (an analog of $\alpha$ for the spinful case) which determines the interactioninduced correction to the conductivity $\Delta \sigma_{\mathrm{AA}}$, decreases for weak interaction $(\alpha \ll 1)$ due to the disorder-induced renormalization of interaction.

For completeness, let us also consider the RG equations for arbitrary strength of interaction. Expanding $\tilde{K}$ in $\mathcal{D}$ and using Eqs. (4.2) and (4.14), we obtain

$$
\tilde{K} \simeq K-\left(1+K^{2}\right) \mathcal{D} / 4 .
$$

Substituting Eq. (4.24) in Eqs. (4.15) and (4.17), we arrive at

$$
\begin{aligned}
\frac{d K}{d \ell} & =-\frac{1}{2}\left[K^{2}-\frac{\left(1+K^{2}\right)(3-2 K)}{2}\right] \mathcal{D}, \\
\frac{d \mathcal{D}}{d \ell} & =(3-2 K) \mathcal{D} .
\end{aligned}
$$

The solution to Eqs. (4.25), (4.26) is shown in Fig. [1. for comparison, we show also the solution to 

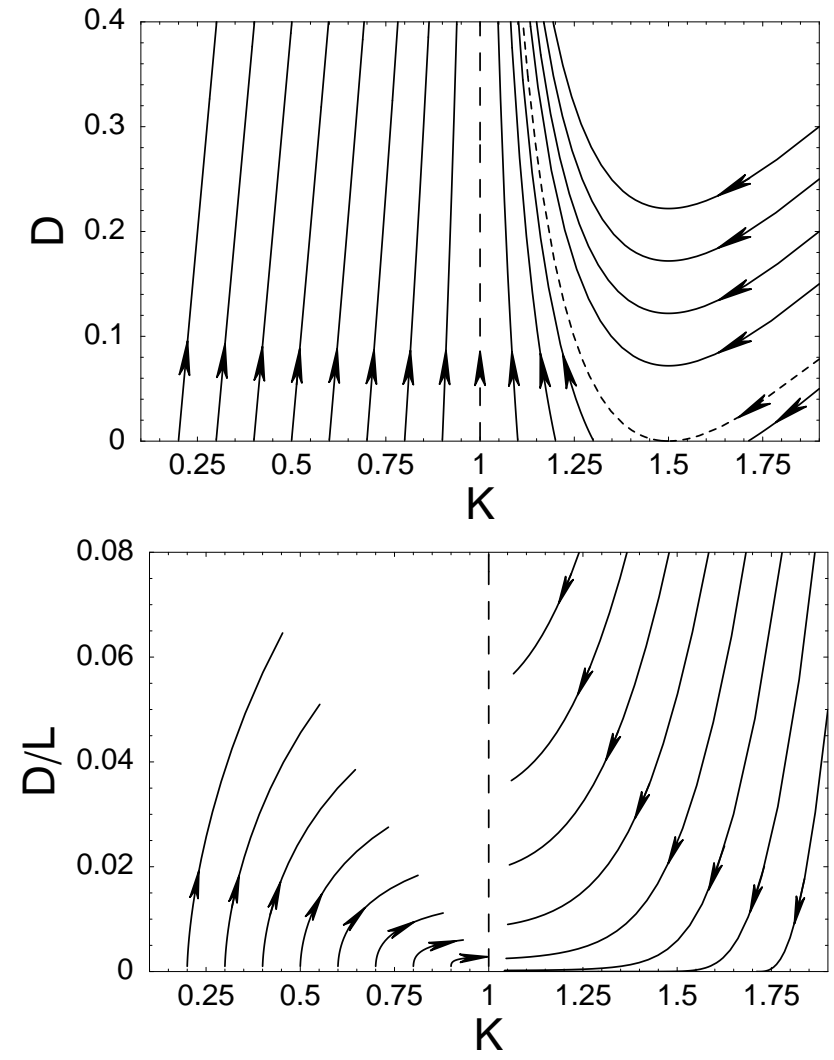

FIG. 1: Upper panel: the RG flows given by Eqs. (4.25), (4.26) in the plane $(K, \mathcal{D})$, where $K$ is the true interaction constant and $\mathcal{D}$ is the strength of disorder (the dimensionless resistance). Dashed line: the separatrix touches the quantum critical point at $K=3 / 2, \mathcal{D}=0$. Long-dashed line: the line of $K=1$ separates the repulsion $(K<1)$ and attraction $(K>1)$ regions. Lower panel: the RG flows from Eqs. (4.25), 4.26) in the coordinates $K$ and $\mathcal{D} / L$, the latter corresponds to the resistivity. The renormalization for each curve is stopped at $\mathcal{D}=1$ (the strong coupling regime). The dashed line separates the repulsion and attraction regions: an attractive (repulsive) interaction cannot become repulsive (attractive) in the course of the renormalization. Initial couplings in the upper panel from left to right: $\mathcal{D}_{0}=0.001$, $K_{0}=0.2,0.3,0.4,0.5,0.6,0.7,0.8,0.9,1.0,1.1,1.2,1.3$ and $K_{0}=1.9, \quad \mathcal{D}_{0}=0.3,0.25,0.2,0.15,0.05$. Separatrix: $K_{0}=1.9 ; \mathcal{D}_{0} \simeq 0.079$. Initial couplings in the lower panel: $K<1$ (from left to right): $\mathcal{D}_{0}=$

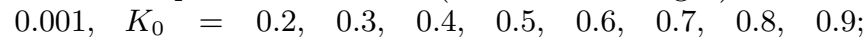
$K>1$ (from top to bottom): $K_{0}=1.9, \mathcal{D}_{0}=$ $0.4,0.35,0.3,0.25,0.2,0.15,0.10,0.05$.

Eqs. (4.15), (4.17) in Fig. 2 The flow equation (4.25) for $K$ tells us that the repulsive interaction, $K<1$, gets weaker in the course of renormalization, $d K / d \ell>0$, whatever the interaction strength is, see Fig. 1, Equations (4.25), (4.26) reduce to the set of Eqs. (4.15), (4.17) around $K=3 / 2$, where the Giamarchi-Schulz RG predicts the metal-insulator transition. Note that for $K>$ $3 / 2$ the flows in terms of $K$ and $\tilde{K}$ are qualitatively similar to each other, as is evident from a comparison of
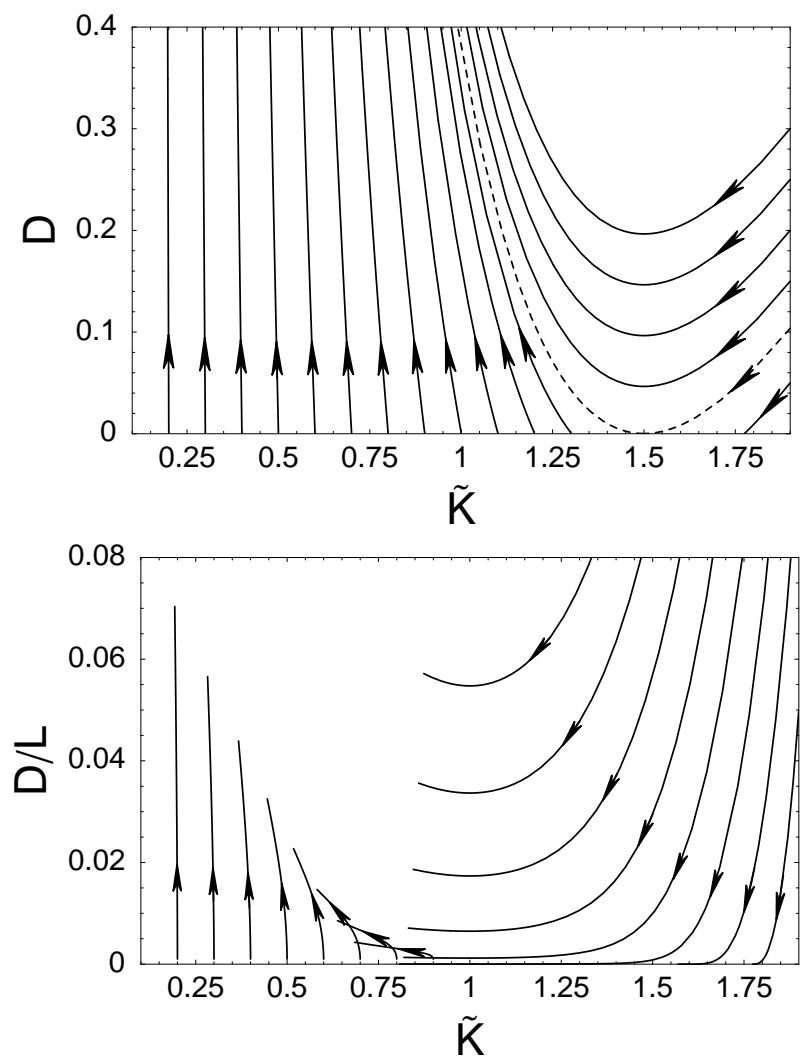

FIG. 2: Upper panel: the RG flows given by Eqs. (4.15), 4.17) in the plane of the modified interaction constant $\tilde{K}$, introduced in Ref. 34, and the strength of disorder (the dimensionless resistance) $\mathcal{D}$. Dashed line: the separatrix touches the quantum critical point at $\tilde{K}=3 / 2, \mathcal{D}=$ 0 . Lower panel: the RG flows from Eqs. (4.15), (4.17) in the coordinates $\tilde{K}$ and $\mathcal{D} / L$ (corresponding to the resistivity). The renormalization for each curve is stopped at $\mathcal{D}=1$ (the strong coupling regime). Initial couplings in the upper panel from left to right: $\mathcal{D}_{0}=0.001, \tilde{K}_{0}=$

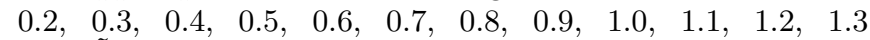
and $\tilde{K}_{0}=1.9, \mathcal{D}_{0}=0.3,0.25,0.2,0.15,0.05$. Separatrix: $\tilde{K}_{0}=1.9 ; \mathcal{D}_{0} \simeq 0.104$. Initial couplings in the lower panel: $\mathcal{D}_{0}=0.001, \tilde{K}_{0}=0.2,0.3,0.4,0.5,0.6,0.7,0.8,0.9$ and $\tilde{K}_{0}=1.9, \mathcal{D}_{0}=0.4,0.35,0.3,0.25,0.2,0.15,0.10,0.05$.

Figs. 1 and 2 .

Let us return to the case of weak interaction and analyze the RG flow generated by Eqs. (4.22), (4.23), see Fig. 3. An approximate solution of these equations in the range of weak disorder, $\mathcal{D}(\ell) \ll 1$ (which is where they are valid), is easily found by an iterative procedure. The result is:

$$
\begin{aligned}
& \mathcal{D}(\ell) \simeq \mathcal{D}_{0}(L / \lambda)^{1+2 \alpha_{0}}, \\
& \alpha(\ell) \simeq \alpha_{0}[1-3 \mathcal{D}(\ell) / 2],
\end{aligned}
$$

where $\mathcal{D}_{0}$ and $\alpha_{0}$ are the initial (ultraviolet) values of the coupling constants at $L \sim \lambda$.

It is worth emphasizing that whereas the correction to $\alpha_{0}$ is of the order of $\alpha_{0}$ itself when $\mathcal{D}(\ell) \sim 1$, the renor- 

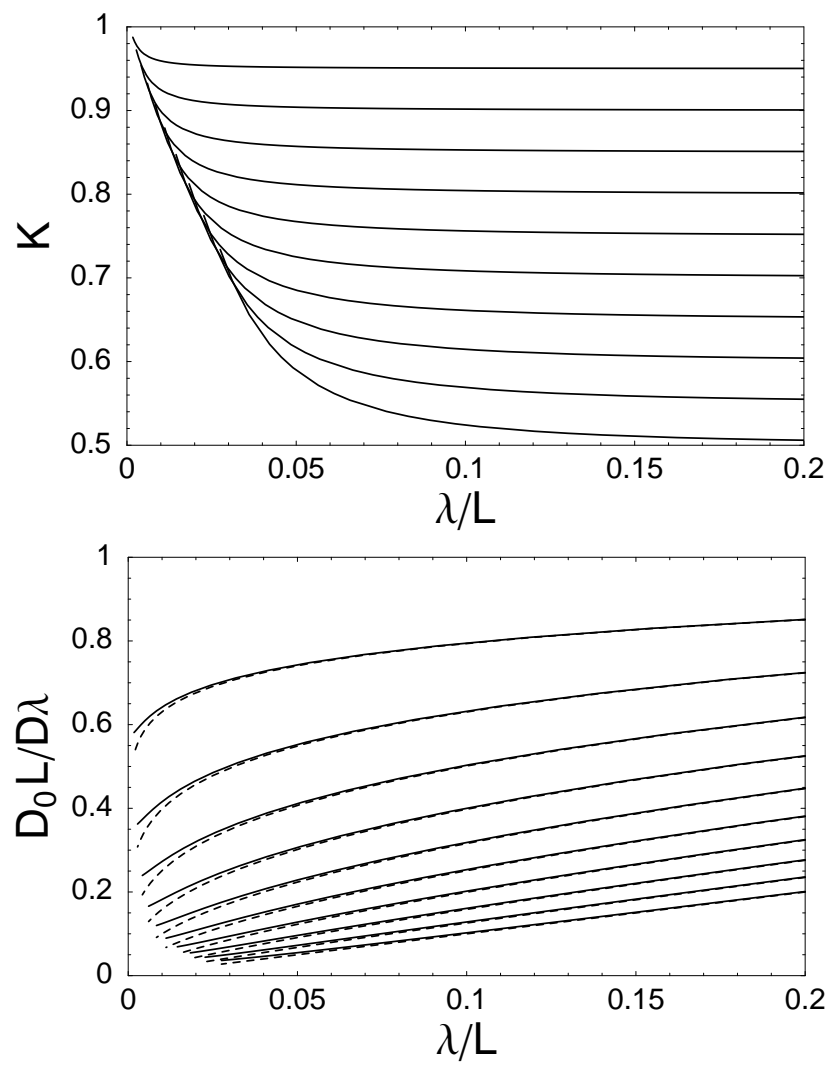

FIG. 3: Upper panel: the renormalization of the interaction constant $K$ due to disorder for various initial values of $K$ in the ultraviolet limit $\lambda / L=1$. The strength of interaction decreases upon renormalization, $K \rightarrow 1$. Lower panel: the renormalization of the "conductivity" $L / \mathcal{D}$ in units of the bare "conductivity" $\lambda / \mathcal{D}_{0}$ (solid line). The dashed lines describe the dependence of $L / \mathcal{D}$ given by Eq. (4.27) with the exponent $\alpha_{0}=1-K_{0}$, where $K_{0}$ is the ultraviolet value of the Luttinger constant. Each flow is stopped at $\mathcal{D}=1$. Initial couplings from top to bottom, both panels: $\mathcal{D}_{0}=0.001$, $K_{0}=0.95,0.9,0.85,0.8,0.75,0.7,0.65,0.6,0.55,0.5$.

malization of disorder is governed by the initial value of the interaction constant and not by the running coupling constant $\alpha(\ell)$. The renormalization of the interaction constant is irrelevant in the present case, and hence the renormalization of disorder reduces to the renormalization of an individual impurity. As a consequence, the exponent $a$ in Eq. (3.17) for the renormalized scattering time $\tau(T)$ is given by the bare interaction constant (the one in a clean system), $a=a_{0} \simeq \alpha_{0}$. Thus, in the Drude formula (3.16) the exponent of the Luttingerliquid power-law function $(T / \Lambda)^{a}$ is $T$-independent.

\section{Bosonization vs Localization}

As discussed in Sec.IVB the solution of the RG equations flows to the strong-coupling regime, where $\mathcal{D} \gtrsim 1$ and the perturbative-in-disorder RG equations are no longer valid. Since in the absence of interaction the strong-coupling fixed point $\mathcal{D} / L \rightarrow \infty$ corresponds to the complete Anderson localization, Giamarchi and Schulz identified the regime $\mathcal{D} \gg 1$ as the localized phase.

It should be stressed, however, that the notion of Anderson localization does not follow from the RG equations of Ref. 34. Indeed, Eq. (4.23) does not account for the renormalization of disorder $\mathcal{D} / L$ in the absence of interaction: the effects of Anderson localization (arising due to interference terms which involve higher powers of $\mathcal{D}$ ) are not included in this RG scheme. In the weak-coupling regime, $\mathcal{D} \ll 1$, the Anderson localization would show up in an additional $\alpha$-independent term, $f_{\mathrm{wl}}(\mathcal{D}) \sim \mathcal{D}^{3}$ on the right-hand side of Eq. (4.23), which is beyond the accuracy of the derivation of these equations. It is also worth noting that in the presence of interaction all the RG trajectories for $\mathcal{D} / L$ tend to saturate at finite values of $\mathcal{D} / L$, as can be seen from Fig. 1, in contrast to what one expects for the Anderson-localization behavior. The strong-coupling fixed point $\mathcal{D} \rightarrow \infty$ is therefore a manifestation of Ohm's law, rather than of the Anderson localization.

In order to study the temperature behavior of $\sigma(T)$, the renormalization procedure in Ref. 34 was stopped at $L=L_{T} \sim u / T$, with the argument that the coherence effects disappear on length scales greater than $L_{T}$ due to inelastic processes. Although this is perfectly correct for the interaction-induced renormalization of disorder which is cut off by the thermal smearing of the electron distribution function [similarly to the Altshuler-Aronov correction to the conductivity in higher- $d$ systems, see Eq. (2.2)], the coherence effects relevant to the Anderson localization are governed by the dephasing length, $L_{\phi}$. The condition $L_{T} \sim \xi$ yielding the temperature $\sim u / \xi$ for the onset of the Anderson localization is in general not correct.

Indeed, the additional term $f_{\mathrm{wl}}(\mathcal{D})$ responsible for the WL (quantum-interference) effects is cut off not by $L_{T}$ but rather by the dephasing length $L_{\phi}$, which is parametrically different from $L_{T}$. In particular, in the absence of interaction $L_{\phi}$ diverges, while $L_{T}$ remains finite, being independent of $\alpha$. For weak interaction $L_{\phi} \gg L_{T}$ and hence, there exists an intermediate range of length scales, $L_{T} \lesssim L \lesssim L_{\phi}$, in which only the WL term $f_{\mathrm{wl}}(\mathcal{D})$ contributes to the renormalization of $\mathcal{D}$. Thus, it turns out that the scalings with $T$ and $L$ are not interchangeable, when $\sigma(T)$ is considered. For $\alpha \ll 1$, the onset of strong localization is determined by the condition

$$
L_{\phi} \sim \xi
$$

which in $1 \mathrm{~d}$ is equivalent to

$$
\tau_{\phi} \sim \tau
$$

After the RG transformation, the bosonization action retains its original form (4.4)-(4.6), but with a renormalized ultraviolet cutoff, $\Lambda \rightarrow T$, and a renormalized scattering rate, $\tau_{0}^{-1} \rightarrow \tau^{-1}(T)$. Performing a transformation 
from the bosons back to fermions, we thus return to the original fermionic theory with a corresponding renormalization of its parameters. This theory is analyzed in the following sections.

\section{GOLDEN RULE IN LUTTINGER LIQUID}

In order to analyze the effects of dephasing, we will follow the route suggested by earlier works on higherdimensional (in particular, diffusive) systems $\underline{46}$ Since the physics of dephasing is governed by electronic inelastic scattering processes, a natural first step is to calculate the e-e scattering rate $\tau_{\mathrm{ee}}^{-1}$. Indeed, for higherdimensional systems in the high-temperature (ballistic) regime, $T \tau \gg 1$, the dephasing rate $\tau_{\phi}^{-1}$ to leading order is given by $\tau_{\text {ee }}^{-1}$, see Ref. 99 . For quasi-1d and 2 d systems in the diffusive regime the scattering kernel acquires an infrared singularity leading to a divergent $\tau_{\mathrm{ee}}^{-1}$. However, even in this case the calculation of the e-e collision rate turns out to be instructive: a parametrically correct result for the dephasing rate can be obtained from the expression for $\tau_{\text {ee }}^{-1}$ supplemented with an appropriate infrared cutoff. It is thus useful to begin by analyzing $\tau_{\text {ee }}^{-1}$. We will show that this quantity is finite and meaningful in the interacting $1 \mathrm{~d}$ system (contrary to what one might expect based on the famous non-Fermi-liquid character of the Luttinger liquid). On the other hand, we will demonstrate that, while governing the dephasing of AharonovBohm oscillations, $\tau_{\mathrm{ee}}^{-1}$ does not give the dephasing rate for the WL of spin-polarized electrons.

The Golden Rule expression for the e-e collision rate following from the Boltzmann kinetic equation reads

$$
\begin{aligned}
\frac{1}{\tau_{\mathrm{ee}}(\epsilon)} & =\int d \omega \int d \epsilon^{\prime} \mathcal{K}(\omega) \\
& \times\left(f_{\epsilon-\omega}^{h} f_{\epsilon^{\prime}} f_{\epsilon^{\prime}+\omega}^{h}+f_{\epsilon-\omega} f_{\epsilon^{\prime}}^{h} f_{\epsilon^{\prime}+\omega}\right),
\end{aligned}
$$

where $\mathcal{K}(\omega)$ is the kernel of the e-e collision integral

$$
\begin{aligned}
\operatorname{St}\left\{f_{\epsilon}\right\} & =\int d \omega \int d \epsilon^{\prime} \mathcal{K}(\omega) \\
& \times\left(-f_{\epsilon} f_{\epsilon-\omega}^{h} f_{\epsilon^{\prime}} f_{\epsilon^{\prime}+\omega}^{h}+f_{\epsilon}^{h} f_{\epsilon-\omega} f_{\epsilon^{\prime}}^{h} f_{\epsilon^{\prime}+\omega}\right) \\
& =-\frac{f_{\epsilon}}{\tau_{\mathrm{ee}}(\epsilon)}+\int d \omega \int d \epsilon^{\prime} \mathcal{K}(\omega) f_{\epsilon-\omega} f_{\epsilon^{\prime}}^{h} f_{\epsilon^{\prime}+\omega} .
\end{aligned}
$$

Here $f_{\epsilon}$ is the Fermi distribution function, $f_{\epsilon}^{h}=1-f_{\epsilon}$.

To leading (second) order in interaction, the scattering kernel $K(\omega)$ in a clean Luttinger liquid has the form

$$
K(\omega)=\eta_{s}\left[K_{++}^{H}(\omega)+K_{+-}^{H}(\omega)\right]+K^{F}(\omega),
$$

where

$$
\begin{aligned}
K_{++}^{H} & =g_{4}^{2} \frac{1}{\pi^{3} \rho} \int \frac{d q}{2 \pi}\left[\operatorname{Re} D_{+}(\omega, q)\right]^{2} \\
K_{+-}^{H} & =g_{2}^{2} \frac{1}{\pi^{3} \rho} \int \frac{d q}{2 \pi} \operatorname{Re} D_{+}(\omega, q) \operatorname{Re} D_{-}(\omega, q) \\
K^{F} & =-K_{++}^{H} .
\end{aligned}
$$

Here $K_{++}^{H}$ and $K_{+-}^{H}$ are related to scattering of two electrons from the same or different chiral spectral branches, respectively, $K_{F}$ is the exchange counterpart of $K_{++}^{H}$, $\eta_{s}$ is the spin degeneracy $\left(\eta_{s}=1\right.$ for the spinless case and $\eta_{s}=2$ for the spinful case), and $D_{ \pm}(\omega, q)$ are the two-particle propagators, given by Eq. (A.4) with $i \Omega_{n} \rightarrow \omega+i 0$.

Substituting Eqs. (5.2)-(5.5) in Eq. (5.1), we obtain the lowest-order result for the e-e scattering rate at the Fermi level $(\epsilon=0)$ in terms of the corresponding contributions to the retarded electronic self-energy $\Sigma_{+}^{R}$ defined by $G_{+}^{R}(\epsilon, p)=\left[\epsilon-v_{F} p-\Sigma_{+}^{R}(\epsilon, p)\right]^{-1}$, where $G_{+}^{R}$ is the retarded Green's function for right-movers:

$$
\begin{aligned}
1 / 2 \tau_{\mathrm{ee}} & =-\operatorname{Im} \Sigma_{+}^{R}(0,0), \\
\Sigma_{+}^{R}(0,0) & =\Sigma_{++}^{H}+\Sigma_{+-}^{H}+\Sigma^{F} .
\end{aligned}
$$

The diagrams for $\Sigma_{+-}^{H}, \Sigma_{++}^{H}$, and $\Sigma^{F}$ are shown in Figs. 4a, $4 \mathrm{~b}$, and $4 \mathrm{k}$, respectively. The contribution of the diagram in Fig. 4b (Hartree term) is canceled by the diagram in Fig. 4r (Fock term) due to the Pauli principle,

$$
\Sigma^{F}=-\Sigma_{++}^{H} .
$$

We refer to this cancellation as the "Hartree-Fock cancellation".

a)

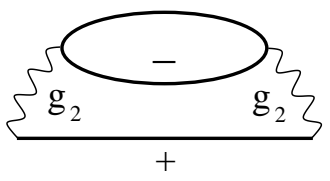

b)

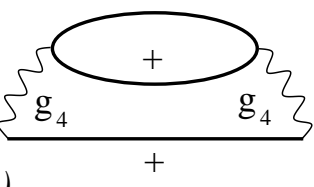

FIG. 4: Second-order ("Golden Rule") diagrams for the selfenergy of a right-mover. Wavy lines: interaction of the rightmover with left- $\left(g_{2}\right)$ or right- $\left(g_{4}\right)$ movers. (a) $\Sigma_{+-}^{H}$, the Hartree contribution due to the interaction with a left mover; (b) $\Sigma_{++}^{H}$, the Hartree contribution due to the interaction with a right mover; (c) $\Sigma^{F}$, the Fock (exchange) contribution.

Let us now calculate the imaginary parts of the perturbative self-energies. Using Eq. (A.4) to get the retarded propagators $D_{ \pm}(\omega, q)$ by analytically continuing $i \Omega_{n} \rightarrow \omega+i 0$ and putting $g_{2}=g_{4}=2 \pi v_{F} \alpha$ we find:

$$
\begin{aligned}
& \operatorname{Im} \Sigma_{++}^{H}=-2 \pi^{2} \alpha^{2} v_{F}^{3} \int \frac{d \omega}{2 \pi} \omega\left(\operatorname{coth} \frac{\omega}{2 T}-\tanh \frac{\omega}{2 T}\right) \\
& \times \int \frac{d q}{2 \pi}\left[\operatorname{Re} D_{+}(\omega, q)\right]^{2} \simeq-\pi \alpha^{2} v_{F} T \\
& \times \int_{|\omega| \lesssim T} d \omega \int d q \delta\left(\omega-v_{F} q\right) \delta\left(\omega-v_{F} q\right)
\end{aligned}
$$




$$
\begin{aligned}
& \operatorname{Im} \Sigma_{+-}^{H}=-2 \pi^{2} \alpha^{2} v_{F}^{3} \int \frac{d \omega}{2 \pi} \omega\left(\operatorname{coth} \frac{\omega}{2 T}-\tanh \frac{\omega}{2 T}\right) \\
& \times \int \frac{d q}{2 \pi} \operatorname{Re} D_{+}(\omega, q) \operatorname{Re} D_{-}(\omega, q) \simeq-\pi \alpha^{2} v_{F} T \\
& \times \int_{|\omega| \lesssim T} d \omega \int d q \delta\left(\omega-v_{F} q\right) \delta\left(\omega+v_{F} q\right) .
\end{aligned}
$$

One sees that the contribution of $\Sigma_{++}^{H}$ is diverging. For spin-polarized electrons (i.e., for $\eta_{s}=1$ ) it is, however, exactly canceled by the exchange interaction, Eq. (5.8). Indeed, as we have discussed in Sec. IIA the $g_{4}$ interaction drops out of the problem in this case, inducing only a shift of the velocity, Eq. (3.12). The remaining term $\Sigma_{+-}^{H}$ is determined by $\omega, q \rightarrow 0$ and is given by

$$
\operatorname{Im} \Sigma_{+-}^{H}=-\pi \alpha^{2} T / 2 .
$$

Already the perturbative expansion demonstrates a qualitative difference between two cases of spinless and spinful electrons - the spinful case will be considered in Ref. 44.

The characteristic frequencies in Eqs. (5.9), (5.10) satisfy $|\omega| \lesssim T$, because of the factor $\operatorname{coth}(\omega / 2 T)-$ $\tanh (\omega / 2 T)$, even if we would perform this calculation in the original theory with the ultraviolet cutoff $\Lambda$. For the sake of comparison, we present also the corresponding contribution to the real part of the self-energy:

$$
\begin{aligned}
& \operatorname{Re} \Sigma_{+}(\epsilon, p)=-2 \pi^{2} \alpha^{2} v_{F}^{2} \int \frac{d \omega}{2 \pi} \int \frac{d q}{2 \pi} \\
& \times\left\{\operatorname{coth} \frac{\omega}{2 T} \frac{q}{\epsilon+\omega-v_{F}(p+q)} \delta\left(\omega+v_{F} q\right)\right. \\
& \left.\quad+\tanh \frac{\omega+\epsilon}{2 T} \frac{q}{\omega+v_{F} q} \delta\left[\epsilon+\omega-v_{F}(p+q)\right]\right\},
\end{aligned}
$$

which for $T \gg\left|\epsilon \pm v_{F} p\right|$ gives

$$
\operatorname{Re} \Sigma_{+}(\epsilon, p) \simeq-\frac{1}{2} \alpha^{2}\left(\epsilon-v_{F} p\right) \ln \frac{\Lambda}{T} .
$$

In contrast to the imaginary part of the self-energy, the real part is determined by energy transfers $|\omega| \gtrsim T$, which is characteristic of the elastic virtual processes. Furthermore, $\operatorname{Re} \Sigma_{+}$is logarithmically divergent in the ultraviolet and is cut off by $\Lambda$ : this behavior is specific to $1 \mathrm{~d}$.

It is worth stressing that in the considered case of weak interaction, $\alpha \ll 1$, the lowest-order contribution to the inelastic scattering rate obtained from Eqs. (5.6),(5.11),

$$
\tau_{\mathrm{ee}}^{-1}=\pi \alpha^{2} T
$$

is much smaller than temperature:

$$
T \tau_{\text {ee }} \gg 1 \text {. }
$$

In higher dimensionalities, this fact is commonly referred to as one of the conditions of the existence of Fermi liquid: the inverse lifetime of a quasiparticle is smaller than its characteristic energy. In this respect, the weakly interacting Luttinger liquid, while being a canonical example of a non-Fermi liquid, reveals the typical Fermi-liquid property. The Luttinger-liquid physics is in fact encoded in the singular real part of the perturbative self-energy, Eq. (5.12). For $\alpha^{2} \ln (\Lambda / T) \ll 1$, the Green's function can be written as $G_{+}^{R}(\epsilon, p) \simeq Z /\left(\epsilon-v_{F} p+i \pi \alpha^{2} T / 2\right)$, where the single-particle weight $Z \simeq 1-\left(\alpha^{2} / 2\right) \ln (\Lambda / T)$ is suppressed by interaction. It is in fact the product $\alpha^{2} \ln (\Lambda / T)$, coming from the real part of the self-energy, that after resummation of all orders gets exponentiated and leads to the Luttinger-liquid power-law singularities. Those singularities have been accounted for by the renormalization at step 1 in the present approach.

It is instructive to compare the perturbative collision rate in a clean Luttinger liquid, Eq. (5.13), with the damping ${ }^{100}$ of the exact retarded single-particle Green's function in the coordinate-time $(x, t)$ representation,

$$
g_{ \pm}^{R}(x, t)=2 i \Theta(t) \operatorname{Im} \bar{g}_{ \pm}(x, t)
$$

Considering right-movers for definiteness, we have

$$
\begin{aligned}
\bar{g}_{+}(x, t) & =(\Lambda / 2 \pi u) A^{1+\alpha_{b} / 2}(x / u-t+i / \Lambda) \\
& \times A^{\alpha_{b} / 2}(x / u+t-i / \Lambda)
\end{aligned}
$$

where

$$
A(z)=\pi T / \Lambda \sinh (\pi T z)
$$

and $\alpha_{b}=\left(K+K^{-1}-2\right) / 2$, which becomes $\alpha_{b} \simeq \alpha^{2} / 2$ for $\alpha \ll 1$.

The Green's function is peaked at the "classical trajectory" $x=u t$. In the limit of large $t \rightarrow \infty$ we have an exponential decay 100

$$
\bar{g}_{+}(x=u t, t) \simeq \frac{-i \Lambda}{2 \pi u}\left(\frac{2 \pi T}{i \Lambda}\right)^{\alpha_{b} / 2} \exp \left(-\pi \alpha_{b} T t\right),
$$

which should be contrasted with a power-law $t$ dependence at zero temperature,

$$
\bar{g}_{+}(u t, t)=\frac{-i \Lambda}{2 \pi u} \frac{1}{(2 i \Lambda t)^{\alpha_{b} / 2}} .
$$

The large factor $\Lambda$ in the numerator of Eq. (5.18) is reminiscent of the pole in the noninteracting Green's function. It is worth stressing that, since $|A(i / \Lambda)| \rightarrow 1$, the nonanalytical singularity of the factor $\sinh ^{-\alpha_{b} / 2}[\pi T(x / u-$ $t+i / \Lambda)]$ in the main peak at $x=u t$ is exactly canceled by the Luttinger-liquid renormalization factor $(T / \Lambda)^{\alpha_{b} / 2}$ which arises due to the resummation of the real part of the self-energy. Thus the exponential decay in Eq. (5.18) comes solely from the factor $[A(2 t-i / \Lambda)]^{\alpha_{b} / 2}$ which describes the tail of the "left-moving peak" centered at $x=-u t$. The decay $\exp \left(-\pi \alpha_{b} T t\right)$ of the "residue"

$$
\left.[x-u(t-i / \Lambda)] g_{+}^{R}(x, t)\right|_{x \rightarrow u t} \propto \sinh ^{-\alpha_{b} / 2}(2 \pi T t)
$$

for $t \rightarrow \infty$ agrees with the Golden Rule expression (5.13) to order $\alpha^{2}: \pi \alpha_{b} T \simeq 1 / 2 \tau_{\text {ee }}$. Therefore, the temporal decay of the Green's function is determined by the processes of inelastic scattering of right-movers on left-movers. 
We see that the perturbative result for the scattering rate yields the asymptotic behavior of the exact Green's function. This means that the Golden Rule calculation of the inelastic scattering time is meaningful in Luttinger liquid. However, the Golden Rule time $\tau_{\text {ee }}$ does not cut off the WL correction in the spinless case, as we demonstrate below in Sec. VI

\section{WEAK LOCALIZATION AND DEPHASING: FERMIONIC PATH-INTEGRAL APPROACH}

\section{A. WL dephasing vs Golden Rule}

The notion of dephasing associated with the behavior of the single-particle Green's function (5.20) makes sense in a clean Luttinger liquid in the ring geometry, see Sec. VIE. However, as we are going to show in this section, the WL dephasing time $\tau_{\phi}^{\mathrm{wl}}$ that governs the cutoff of the WL correction $\Delta \sigma_{\mathrm{wl}}$ is parametrically different from the Golden Rule time $\tau_{\text {ee }}$, Eq. (5.13).

This difference can in fact be anticipated based on the following observation. The integral (5.10) for the Golden Rule rate is determined by the energy and momentum transfer $q, \omega=0$. On the other hand, in the spirit of Ref. [58, soft inelastic scattering with $q v_{F}, \omega \ll 1 / \tau_{\phi}^{\mathrm{wl}}$ is not expected to contribute to the WL dephasing. This suggests that the dephasing rate $1 / \tau_{\phi}^{\mathrm{wl}}$ requires a selfconsistent cutoff in Eq. (5.1) at $q v_{F}, \omega \sim 1 / \tau_{\phi}^{\mathrm{wl}}$, similarly to Eq. (2.4) for higher-dimensional systems, and so is parametrically different from the one in Eq. (5.13). In particular, if one introduces, similarly to higher dimensionalities, a sharp low-frequency cutoff in the right-hand side of Eq. (5.10), one immediately gets zero instead of Eq. (5.13). On the other hand, introducing disorder in $D_{+}$and $\Pi_{-}$in Eq. (5.10), one effectively broadens the delta functions by $1 / \tau$, and the self-consistent equation can be solved.

Such a self-consistent equation should be treated with the utmost caution, in view of the singular character of the ballistic propagators. In particular, when transformed to the space-time representation, the integral in Eq. (5.10) is determined by a single point, $x, t=0$, so that one could be drawn to the erroneous conclusion that it is high - rather than low - frequencies that matter. In any case, this equation is at most qualitative. However, the message that we learn from this consideration is correct: (i) the WL dephasing rate differs from the Golden Rule result, and (ii) to evaluate it, we have to include disorder in the interaction propagators. To perform a controllable quantitative analysis, we employ the pathintegral technique, which is a natural tool in the situation when the diagrammatic calculation suffers from the infrared problems.

\section{B. Weak localization in $1 \mathrm{~d}$}

We begin by identifying the leading contribution to the WL correction to the conductivity in the regime of strong dephasing, $\tau_{\phi}^{\mathrm{wl}} / \tau \ll 1$. This regime takes place at sufficiently high temperatures - the precise condition is found below in Sec. IIIc, where we evaluate $\tau_{\phi}^{\mathrm{wl}}$. The diagrams that give the main contribution to $\Delta \sigma_{\mathrm{wl}}$ are shown in Fig. [5]

a)

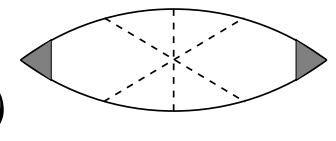

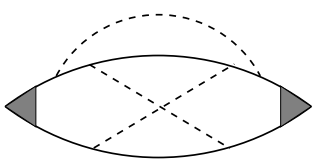

b)

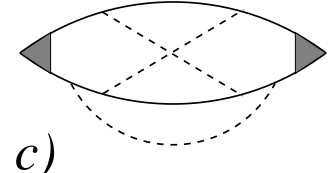

c)
FIG. 5: Diagrams describing the leading WL correction to the conductivity of Luttinger liquid for $\tau_{\phi}^{\mathrm{wl}} \ll \tau$. The dashed lines represent backscattering off impurities. The current vertices are dressed by impurity ladders. The solid lines are the Green's functions with disorder-induced self-energies. The diagrams are understood as fully dressed by e-e interactions.

The "three-impurity Cooperon" in Fig. 5a describes the propagation of two electron waves along the path connecting three impurities ("minimal loop") in timereversed directions, Fig. 6 6 a. Indeed, one can easily check that the interference paths involving only two backscattering impurities are impossible merely due to geometrical reasons. This means that the Cooperon with two backscattering impurity lines 101 yields zero contribution to the conductivity to leading order in $\left(\epsilon_{F} \tau\right)^{-1}$. If one retains the forward scattering and does not gauge it out, the two-impurity Cooperon diagram (Fig. $7 \mathrm{~g}$ ) is canceled by the contribution of two other non-Drude diagrams with two impurity lines (Fig. 7b,c). The diagrams represented in Fig. 5 b,c (the corresponding trajectories are shown in Fig. 6b) are of the same order as the Cooperon diagram.

In the absence of interaction, all higher-order diagrams (including non-Cooperon diagrams) which describe quantum-interference processes involving a larger number of impurities $(4,5, \ldots)$ are equally important and together with the diagrams in Fig. 5 sum up to exactly cancel $\sigma_{\mathrm{D}}$, which spells a complete localization. $\underline{69}$ In the WL regime, they only yield subleading corrections through a systematic expansion in powers of the small parameter $\tau_{\phi}^{\mathrm{wl}} / \tau$. Thus, the WL in $1 \mathrm{~d}$ is governed by the "shortest possible Cooperon", namely, the Cooperon with three impurity lines. In essence, this is due to the fact that the WL condition $1 / \tau_{\phi}^{\mathrm{wl}} \gg \Delta_{\xi}$ reduces in $1 \mathrm{~d}$ to the condition of the "ballistic dephasing", $\tau_{\phi}^{\mathrm{wl}} / \tau \ll 1$. In turn, this means that the contribution to $\Delta \sigma_{\mathrm{wl}}$ of typical 

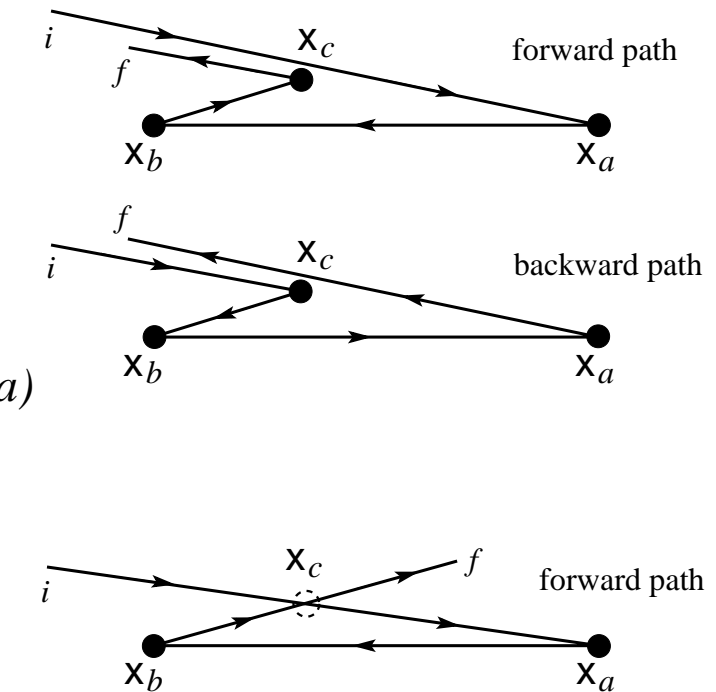

b)

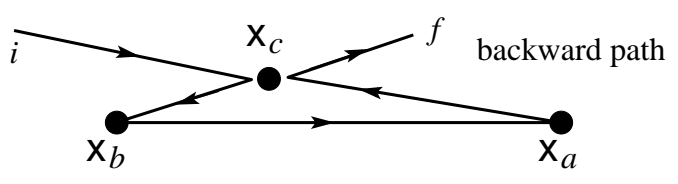

FIG. 6: (a) Interference paths described by the Cooperon diagram, Fig. 5 a. Black dots: the backscattering off impurities. The one-dimensional graphs are stretched vertically for ease of visualization. The two waves start at point $i$ and interfere at point $f$ after traversing the closed loop in opposite directions. (b) Interfering paths for the diagram in Fig. 5. The dashed circle denotes the absence of backscattering off the impurity at point $x_{c}$. The paths corresponding to the diagram in Fig. $5 \mathrm{~b}$ are obtained by interchanging the upper and lower plots in Fig. 6b. Note that similar paths describe the WL in the ballistic regime in higher dimensionalities. ${ }^{102}$

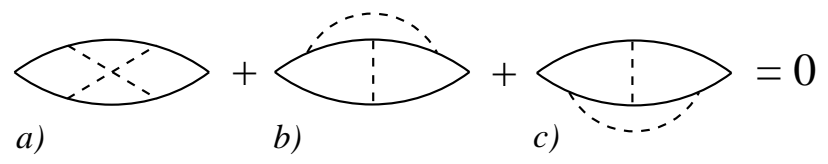

FIG. 7: Two-impurity non-Drude diagrams.

configurations of three impurities separated by a distance of order $v_{F} \tau$ is strongly suppressed by dephasing. In the compact configurations that do contribute the three impurities are anomalously close to each other, with a characteristic distance between them being much smaller than the mean free path.

\section{WL dephasing in 1d: Qualitative discussion of the leading-order cancellation}

Before turning to the path-integral evaluation of the WL dephasing rate in a spinless $1 \mathrm{~d}$ system, we present a simple argument demonstrating the cancellation of the leading contribution $1 / \tau_{\mathrm{ee}}$ to $1 / \tau_{\phi}^{\mathrm{wl}}$. For the purpose of a qualitative discussion, we can consider inelastic scattering within the picture of a classical thermal noise with characteristic frequencies $|\omega| \ll T$. Let $U_{0}^{ \pm}(x)$ be a given realization of the fluctuating scalar potential $U^{ \pm}(x, t=0)$ created by right-/left-movers at time $t=0$. The peculiarity of the $1 \mathrm{~d}$ geometry is that in the clean case the initial density profiles of right-/left-movers, whose dispersion relation is linear, remain unchanged: they move with the plasmon velocity either to the left or to the right as a whole, and so does the fluctuating potential:

$$
U^{ \pm}(x, t)=U_{0}^{ \pm}(x \mp u t) .
$$

We now demonstrate that the phases acquired by the time-reversed waves propagating along a closed threeimpurity loop are equal for arbitrary $U_{0}^{ \pm}(x)$ and hence cancel each other in the interference contribution even before the averaging over fluctuations of $U_{0}^{ \pm}(x)$. As we show in Appendix $\mathrm{F}$ the velocity of electrons moving along the "Cooperon path" is renormalized by the interaction and coincides with $u$. Referring to the paths shown in Fig. 6 6 a, we first follow the "forward" trajectory $x_{c}=0 \rightarrow x_{a} \rightarrow x_{b} \rightarrow 0$ for a particle which starts moving to the right at $t=0$ and is backscattered at these points at times $t_{a}, t_{b}$, and $t_{c}$, respectively, see Fig. 8. It is important to recall that the scattering amplitude $g_{4}$ has been eliminated through the renormalization of the Fermi velocity. Therefore, on the right-moving parts of the forward trajectory (segments $0 \rightarrow x_{a}$ and $x_{b} \rightarrow 0$ ) the phase changes by $\phi_{+}^{F}$ only due to the scattering off $U^{-}(x, t)$, while on the left-moving parts (segment $x_{a} \rightarrow x_{b}$ ) only the scattering off $U^{+}(x, t)$ matters and yields the phase shift $\phi_{-}^{F}$. Similarly, there are phase shifts for the backward path $\phi_{ \pm}^{B}$ due to the scattering off $U^{\mp}(x, t)$.

The phase $\phi_{-}^{F}$ acquired on the forward path between points $x_{a}$ and $x_{b}$ is given by

$$
\phi_{-}^{F}=-\frac{1}{u} \int_{-u t_{c}}^{0} U_{0}^{+}(x) d x .
$$

Here we use $t_{b}-t_{a}=t_{c} / 2$. Electrons moving on the backward path interact with the same right-moving potential $U^{+}(x, t)$ on the segments $0 \rightarrow x_{b}$ and $x_{a} \rightarrow 0$, see Fig. 9. The phase $\phi_{-}^{B}$ is written as

$$
\phi_{-}^{B}=-\frac{1}{u} \int_{-2 u\left(t_{c}-t_{b}+t_{a}\right)}^{0} U_{0}^{+}(x) d x=\phi_{-}^{F} .
$$

Similarly, $\phi_{+}^{F}=\phi_{+}^{B}$. This is clearly seen in Figs. $8 \mathrm{~d}$ and 9d, where the regions of the potential profile that contribute to the phase shifts are identical at time $t_{c}$ for the forward and backward paths. As a result, the interactioninduced phase shifts cancel out in the total Cooperon phase $\left(\phi_{+}^{F}+\phi_{-}^{F}\right)-\left(\phi_{+}^{B}+\phi_{-}^{B}\right)$ for an arbitrary profile $U_{0}(x)$. This means, in particular, that in a calculation of the conductance through a short piece of Luttinger liquid to third order in the impurity strength, which is 


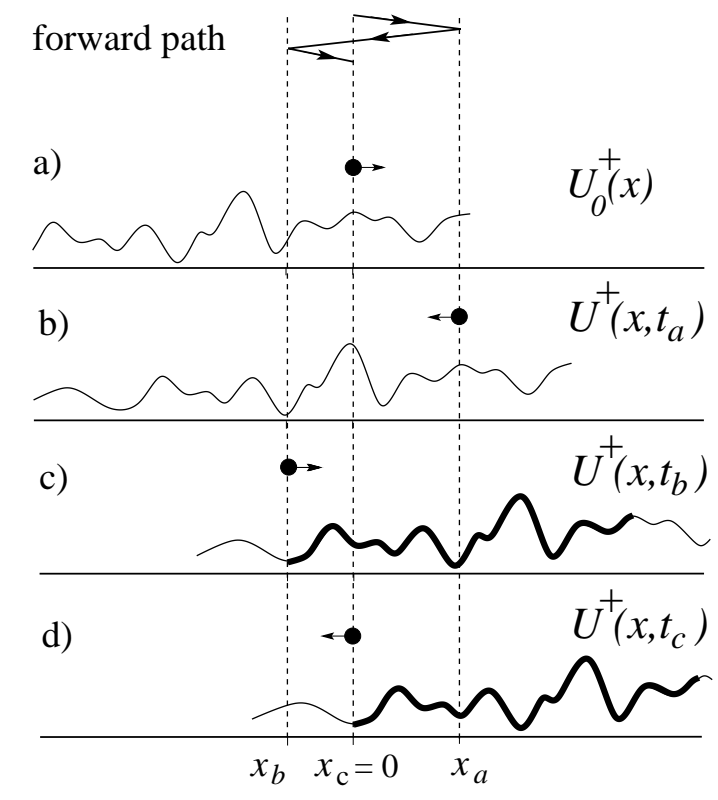

FIG. 8: Illustration of the quasiclassical electron dynamics in the presence of three impurities (whose positions are denoted by the dashed lines) and a right-moving component of the fluctuating scalar potential $U^{+}(x, t)$. Top: the forward path corresponds to that in Fig. 6a. The position (black dots) and the direction of the velocity (arrows) of the particle, as well as the profile of the fluctuating potential, are shown at different times: (a) $t=0$; (b) $t=t_{a}+0$; (c) $t=t_{b}+0$; (d) $t=t_{c}+0$. The phase $\phi_{-}^{F}$ of the electronic wave is accumulated when the particle moves to the left, that is between the snapshots (b) and (c). The segment of the fluctuating potential that contributes to the phase shift is marked in bold.

the order at which the interference term arises, the latter would remain unaffected by inelastic e-e collisions. In the calculation of the WL correction to the conductivity of an infinite quantum wire, we have, indeed, to include disorder in the interaction propagator, despite the ballistic character of dephasing in the WL regime and in accordance with the argument made in Sec. VIA This is done systematically within the path-integral approach in the next subsection.

\section{WL dephasing in 1d: Path-integral calculation}

The dephasing-induced action $S\left(t_{c}, t_{a}\right)$ acquired by the Cooperon is accumulated on the classical (saddle-point) path, whose geometry for three impurities if fixed by two length scales, the total length of the path $v_{F} t_{c}$ and the distance between two rightmost impurities $v_{F} t_{a} \leq$ $v_{F} t_{c} / 2$ (Fig. 10). As shown in Appendix B, the WL correction can then be represented as

$$
\Delta \sigma_{\mathrm{wl}}=-2 \sigma_{\mathrm{D}} \int_{0}^{\infty} d t_{c} \int_{0}^{\infty} d t_{a} P_{2}\left(t_{c}, t_{a}\right) \exp \left[-S\left(t_{c}, t_{a}\right)\right]
$$

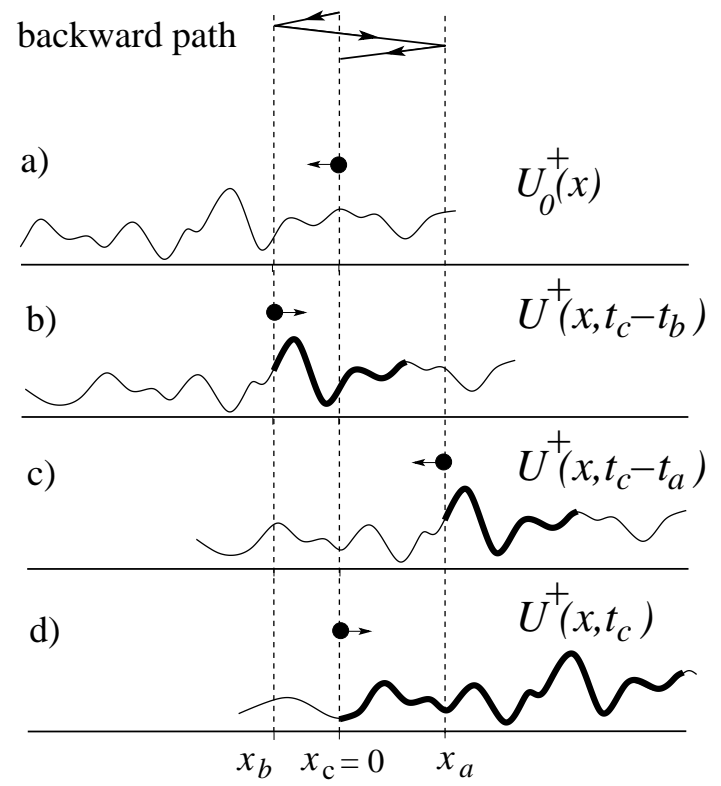

FIG. 9: Same as in Fig. 8 but for the backward part, Fig. 6a. (a) $t=0$; (b) $t=t_{c}-t_{b}+0$; (c) $t=t_{c}-t_{a}+0$; (d) $t=t_{c}+0$. The phase $\phi_{-}^{B}$ of the electronic wave is accumulated when the particle moves between the snapshots (a) and (b) and between the snapshots (c) and (d).

where

$$
P_{2}\left(t_{c}, t_{a}\right)=\left(1 / 8 \tau^{2}\right) \exp \left(-t_{c} / 2 \tau\right) \Theta\left(t_{c}-2 t_{a}\right)
$$

is the probability density of return to point $x=0$ after two reflections at points $x=v_{F} t_{a}$ and $x=-v_{F}\left(t_{c} / 2-t_{a}\right)$ (in this subsection, we ignore the difference between $v_{F}$ and $u$ ). The contribution $S_{i j}$ to the dephasing action associated with inelastic interaction between electrons propagating along the paths $x_{i}(t)$ and $x_{j}(t)$ is obtained similarly to higher dimensionalities: $\stackrel{46,58}{ }$

$$
\begin{aligned}
& S_{i j}=-T \int \frac{d \omega}{2 \pi} \int \frac{d q}{2 \pi} \int_{0}^{t_{c}} d t_{1} \int_{0}^{t_{c}} d t_{2} \frac{1}{\omega} \operatorname{Im} V_{\mu \nu}(\omega, q) \\
& \times \exp \left\{i q\left[x_{i}\left(t_{1}\right)-x_{j}\left(t_{2}\right)\right]-i \omega\left(t_{1}-t_{2}\right)\right\}
\end{aligned}
$$

where $V_{\mu \nu}(\omega, q)$ is the dynamically screened retarded interaction. Because of the HF cancellation of the bare interaction between electrons from the same chiral branch ( $g_{4}$ processes), the screened interaction $V(\omega, q)$ acquires the indices $\mu, \nu$ denoting the direction of motion of the interacting electrons, $\mu=\operatorname{sgn} \dot{x}_{i}, \nu=\operatorname{sgn} \dot{x}_{j}$. More specifically, the $g_{4}$ processes have been incorporated in the renormalization of the bare Fermi velocity. It is worth stressing that if we would keep both $g_{2}$ and $g_{4}$ processes in $V(\omega, q)$, the dephasing action in 1d could not be written in the form of Eq. (6.6). Indeed, in that case the dephasing action would generate the contribution of the diagrams of type (b) in Fig. 4, which is actually absent due to the HF cancellation (5.8).

The main steps in the derivation of Eq. (6.6) are as follows. First, the interaction is treated within the randomphase approximation (RPA), neglecting closed electronic 


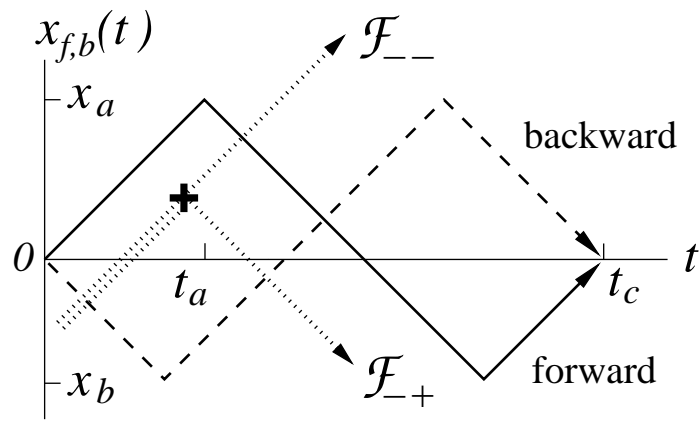

FIG. 10: Illustration of electron dynamics governing the WL and dephasing: Time-reversed paths $x_{f}(t)$ (solid) and $x_{b}(t)=x_{f}\left(t_{c}-t\right)$ (dashed) on which the interaction-induced action $S$ that yields dephasing of the Cooperon is accumulated. Dotted lines: the propagation of dynamically screened interaction. The interaction may change the direction of propagation upon scattering off disorder (as marked by a cross). Each interaction line gives a contribution to $S$ proportional to $\left(N_{f}-N_{b}\right)^{2}$, where $N_{f, b}$ is the number of its intersections with the forward $(f)$ and backward $(b)$ paths. One sees that $N_{f} \neq N_{b}$ only due to impurity scattering in the interaction propagator. Interaction and electron lines lying on top of each other do not yield dephasing because of the HF cancellation.

bubbles with more than two interaction legs, which is justified for the weak interaction, $\alpha \ll 1$. Second, we perform an independent averaging of the RPA bubble and the electronic trajectories over the disorder. This is justified if the characteristic energy transfer $\omega \sim 1 / \tau_{\phi}^{\mathrm{wl}}$ is much larger $1 / \tau$. Further, we treat the thermal electromagnetic fluctuations through which electrons interact with each other as a classical field, which is a valid approximation provided that $\omega$ is much smaller than $T$. As will be seen from the results of our calculation, the characteristic energy transfer $\omega \sim 1 / \tau_{\phi}^{\mathrm{wl}}$ does satisfy both these requirements for the considered case of weak interaction, $\alpha \ll 1$. Finally, we will neglect the influence of the interaction on the velocity ( $u$ vs $v_{F}$ ), which is again justified to the leading order in the interaction strength.

Expanding $V_{\mu \nu}$ to second order in $\alpha$, we have

$$
\operatorname{Im} V_{\mu \nu}(\omega, q)=-\pi \alpha^{2} v_{F} \omega \mathcal{F}_{\mu \nu}(\omega, q),
$$

where the functions $\mathcal{F}_{\mu \nu}$ Fourier-transformed to $(x, \tau)$ space are given by Eqs. (C.2), (C.3) with $\gamma=1 / \tau$. The action $S_{i j}$ can then be written in a simple form:

$S_{i j}=\pi \alpha^{2} v_{F} T \int_{0}^{t_{c}} d t_{1} \int_{0}^{t_{c}} d t_{2} \mathcal{F}_{\mu \nu}\left[x_{i}\left(t_{1}\right)-x_{j}\left(t_{2}\right), t_{1}-t_{2}\right]$,

where, to first order in $\tau^{-1}, \mathcal{F}_{\mu \nu}(x, t)$ read

$$
\begin{aligned}
& \mathcal{F}_{++}(x, t) \simeq \delta\left(x+v_{F} t\right)(1-|t| / 2 \tau), \\
& \mathcal{F}_{+-}(x, t) \simeq \Theta\left(v_{F}^{2} t^{2}-x^{2}\right) / 4 v_{F} \tau,
\end{aligned}
$$

and $\mathcal{F}_{--}(x, t)=\mathcal{F}_{++}(-x, t), \mathcal{F}_{-+}(x, t)=\mathcal{F}_{+-}(x, t)$. The total action is given by $S=2\left(S_{\mathrm{ff}}-S_{\mathrm{fb}}\right)$, where $f$ and $b$ stand for "forward" and "backward" time-reversed paths (Fig. 100).

Let us first calculate $S$ for the case when the disorder in interaction propagators (6.9), (6.10) is neglected, $\tau^{-1}=$ 0 . We get straightforwardly

$$
S_{\mathrm{ff}}=S_{\mathrm{fb}}=\pi \alpha^{2} T t_{c} / 2 .
$$

One sees that $S_{\mathrm{ff}}$ reproduces the Golden Rule result, Eqs. (5.13), (5.20). As we explain in Sec. VIE this rate is relevant for the $\mathrm{AB}$ dephasing in the multiple-connected (ring) geometry. However, in the present case of dephasing in a wire the self-energy processes $\left(S_{\mathrm{ff}}+S_{\mathrm{bb}}\right)$ are exactly canceled in the action by the vertex corrections $\left(S_{\mathrm{fb}}+S_{\mathrm{bf}}\right)$, yielding $S=0$. This confirms the conclusion of Sec. VIC for clean-system interaction propagators, the WL dephasing is absent.

Therefore, the dephasing in Eq. (6.4) is only due to the dressing of the dynamically screened interaction by impurities. A calculation of the dephasing action $S$ to order $\mathcal{O}\left(\tau^{-1}\right)$ is presented in Appendix $[$, with the result

$$
S\left(t_{c}, t_{a}\right)=2 \pi \alpha^{2} T t_{a}\left(t_{c}-2 t_{a}\right) / \tau .
$$

The dephasing vanishes for $t_{a}=0, t_{c} / 2$, since in these cases the two paths $f$ and $b$ are identical.

Substituting Eq. (6.12) in Eq. (6.4) we find for $\tau_{\phi}^{\mathrm{wl}} \ll \tau$ :

$$
\begin{aligned}
& \Delta \sigma_{\mathrm{wl}}=-\frac{1}{4} \sigma_{\mathrm{D}} \int_{0}^{\infty} d t_{c} \frac{1}{\tau^{2}} \exp \left(-t_{c} / 2 \tau\right) \\
& \times \int_{0}^{t_{c} / 2} d t_{a} \exp \left[-4 \pi \alpha^{2} T t_{a}\left(t_{c} / 2-t_{a}\right) / \tau\right] \\
& =-\frac{1}{4} \sigma_{\mathrm{D}}\left(\frac{\tau_{\phi}^{\mathrm{wl}}}{\tau}\right)^{2} \ln \frac{\tau}{\tau_{\phi}^{\mathrm{wl}}} \propto \frac{1}{\alpha^{2} T} \ln \left(\alpha^{2} T\right),
\end{aligned}
$$

where

$$
\frac{1}{\tau_{\phi}^{\mathrm{wl}}}=\alpha\left(\frac{\pi T}{\tau}\right)^{1 / 2}, \quad T \gg T_{1}=\frac{1}{\alpha^{2} \tau} .
$$

In agreement with the above findings, $1 / \tau_{\phi}^{\mathrm{wl}}$ vanishes in the clean limit $\frac{103}{10}$ in contrast to the total e-e scattering rate, Eq. (5.13). The logarithmic factor in Eq. (6.13) is related to the contribution of rare configurations of disorder in which two of the three impurities are anomalously close to each other $\left(t_{a} \rightarrow 0\right.$ or $\left.t_{a} \rightarrow t_{c} / 2\right)$ : in these configurations the dephasing is strongly suppressed. As a result, the characteristic $t$ in the integral (6.13) are spread over the whole range between $\tau_{\phi}^{\mathrm{wl}}$ and $\tau$.

The scale $T_{1}$ defined in Eq. (6.14) marks the temperature below which the localization effects become strong. It is worth mentioning that the $T$ dependence of $\sigma(T)=\sigma_{\mathrm{D}}(T)+\Delta \sigma_{\mathrm{wl}}(T)$ starts to be dominated, with decreasing $T$, by the WL term rather than by $\sigma_{\mathrm{D}}(T)$ at $T \sim T_{1} / \alpha$, i.e., well above $T_{1}$ for $\alpha \ll 1$. These results are illustrated in Fig. 11]

Let us briefly mention what happens below $T_{1}$. In the strongly localized phase, the only peculiarity of Luttinger liquid is the $T$ dependence of the scattering rate 


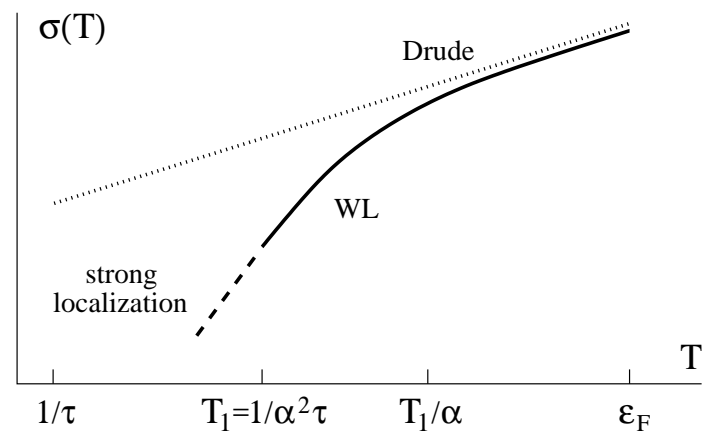

FIG. 11: Schematic behavior of $\sigma(T)$ on the log-log scale. Dotted line: the $T$-dependent Drude conductivity. Below $T_{1} / \alpha$ the WL correction, Eq. (6.13), dominates $d \ln \sigma / d \ln T$. Below $T_{1}$ the localization becomes strong.

$\tau^{-1}(T)$ and the related $T$ dependence of the localization length $\xi(T)$, persisting down to $T \tau(T) \sim 1$ (see Sec. IIIB 1); otherwise, the picture is similar to that in many-channel quantum wires or $2 \mathrm{~d}$ systems. If $\alpha \ll 1$, there exists a wide range of $T$ where the localization is strong but the conductivity is a power-law function of $T$. We call this transport regime in a weakly disordered system, associated with interaction-induced diffusion over localized states, Power-Law Hopping (PLH); see Refs. 65, 70, 104, 105 for details. An important point is that the lifetime of localized states due to inelastic e-e scattering can be calculated in the PLH regime at the Golden Rule level. At still lower $T$, the lifetime becomes infinite, so that the e-e interaction can no longer support hopping via localized states and the conductivity vanishes at a certain critical $T_{c}$, see Refs. 65,66 for details.

\section{E. Weak localization vs Aharonov-Bohm dephasing}

As shown in Sec. VID, the dephasing rate that cuts off the WL correction is much smaller than the Golden Rule result from Sec. $\mathrm{V}$, which governs the damping of the single-particle Green's function, Eq. (5.20). One may ask if the Golden Rule rate $1 / \tau_{\text {ee }}$ is observable at all. The answer is yes: this type of dephasing makes sense in a clean Luttinger liquid in the ring geometry, where it governs the decay rate $\left(\tau_{\phi}^{\mathrm{AB}}\right)^{-1}$ of Aharonov-Bohm $(\mathrm{AB})$ oscillations. ${ }^{45,100,106}$ Indeed, the amplitude $G_{A B}$ of the first harmonic of the $\mathrm{AB}$ conductance is proportional to the product of the Green's functions in two arms of the interferometer:

$$
\begin{aligned}
G_{A B} & \propto\left\{\frac{1}{\Omega_{n}} \int_{0}^{1 / T} d \tau e^{i \Omega_{n} \tau}\right. \\
& \left.\times g_{+}\left(L_{1}, \tau\right) g_{+}\left(-L_{2},-\tau\right)\right\}_{\Omega_{n} \rightarrow+0},
\end{aligned}
$$

where $L_{1,2}$ are the lengths of the arms of the ring. In the case of $L_{1}=L_{2} \equiv L$, the damping is solely due to the dephasing and is given by

$$
G_{A B} \propto \exp \left(-L / u \tau_{\mathrm{ee}}\right) \equiv \exp \left(-L / u \tau_{\phi}^{\mathrm{AB}}\right),
$$

i.e., $\tau_{\phi}^{\mathrm{AB}}=\tau_{\mathrm{ee}}$. The difference from the WL correction is in the absence of the vertex parts of the dephasing action. Specifically, $S_{12}=S_{21}=0$ in the AB setup [where $S_{i j}$ is given by Eq. (6.6) with $i, j$ equal to 1 or 2 for trajectories in arms 1 or 2, correspondingly], because of the absence of interaction between the arms, see Fig. 12, On

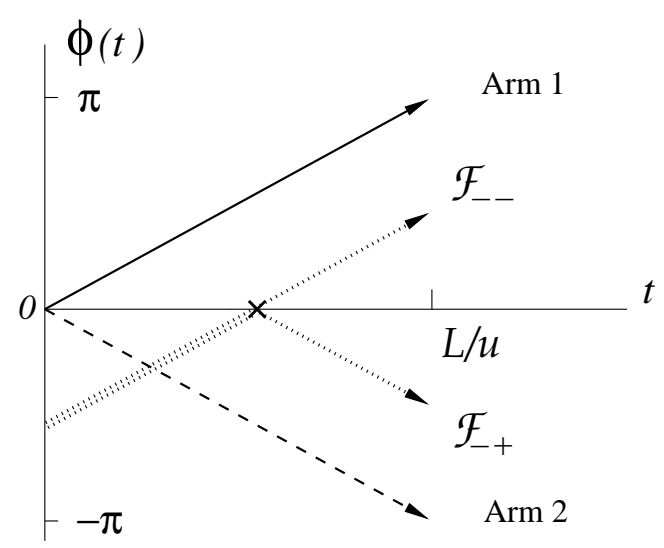

FIG. 12: Illustration of electron dynamics governing the dephasing of the first harmonics $G_{\mathrm{AB}}$ of the $\mathrm{AB}$ oscillations in the ring geometry with two arms of equal length $L$. The position of a particle on the ring is parametrized by the angle $\phi(t)$ : $\phi=0$ corresponds to the point at which the ring is connected to one lead, $\phi= \pm \pi$ - to the point at which it is connected to the other lead. The paths $\phi_{1}(t)$ (solid line) and $\phi_{2}(t)$ (dashed) traveling along arm 1 and arm 2 , respectively, interfere at the second contact at time $t=L / u$. Dotted lines: the propagation of the dynamically screened interaction. The interaction may change the direction of propagation upon scattering off the contact (as marked by a cross). Similarly to Fig. 11] each interaction line gives a contribution to the AB-dephasing action proportional to $\left(N_{1}-N_{2}\right)^{2}$, where $N_{1,2}$ is the number of its intersections with the trajectory in arm 1 or arm 2. One sees that for any dotted line the number of possible intersections in the time domain $0<t<L / u$ is strictly one, yielding only the "self-energy" contribution to the action and no "vertex part". Interaction and electron lines lying on top of each other do not contribute to the dephasing because of the $\mathrm{HF}$ cancellation.

the other hand, the self-energy part $S_{11}=S_{22}$ (analog of $S_{\mathrm{ff}}$ and $S_{\mathrm{bb}}$ in the WL problem) is given by Eq. (6.11) with $t_{c}=L / u$. An analogous $T^{-1}$ dependence of $\tau_{\phi}^{\mathrm{AB}}$ was also obtained in Ref. 107 for a quantum wire coupled capacitively to metallic gates. The preexponential factor in Eq. (6.15) depends on details of how the $\mathrm{AB}$ ring is connected to the leads. A particular AB setuptwo Luttinger liquids weakly coupled to each other at two points by tunneling - was thoroughly studied in Ref. 106. To summarize, the $\mathrm{WL}$ and $\mathrm{AB}$ dephasing rates in a Luttinger liquid are parametrically different in the spinless case studied in this paper; specifically, $\tau_{\phi}^{\mathrm{AB}} \ll \tau_{\phi}^{\mathrm{wl}}$. In diffusive many-channel wires, an analogous difference was shown in Ref. 108. 


\section{FUNCTIONAL BOSONIZATION: ALL-IN-ONE APPROACH}

Equations (6.13), (6.14) have been derived in two steps, emphasizing two key ingredients of the theory: the Luttinger-liquid renormalization of disorder and the Fermi-liquid-like dephasing of electrons scattered by the renormalized disorder. Although both effects are generated by e-e interactions, they are of essentially different origin: the renormalization of disorder is due to virtual processes with energy transfers in the range between $T$ and $\Lambda$, whereas the dephasing stems from real processes with energy transfers smaller than $T$. Conceptually, an advantage of the two-step approach is that it reveals the transparent physical picture of transport in the disordered Luttinger liquid. Alternatively, one can develop a unified approach where the virtual and real transitions are treated on an equal footing, which is what we do below. A fully bosonized approach, as well as a purely fermionic one, turns out to be not particularly advantageous for this purpose. Instead, in this paper we employ the method of so-called "functional bosonization", adjusted to deal with the particulars of e-e scattering in one spatial dimension. The method was introduced for a clean Luttinger liquid by Fogedby ${ }^{89}$ and by Lee and Chen .90 A transparent exposition and further development of the method in $1 \mathrm{~d}$ can be found in recent reviews by Yurkevich ${ }^{92}$ and Lerner and Yurkevich. ${ }^{93}$ For recent applications to single-impurity problems see Refs. 109.110.

In essence, the functional bosonization is a technique based on the conventional Hubbard-Stratonovich transformation that decouples the density-density interaction term in the fermionic action. Bosonic fields appear in this approach as auxiliary fields of the HubbardStratonovich transformation, whose fluctuations mediate the dynamically screened interaction between electrons. The peculiarity of a clean Luttinger liquid is that the RPA approximation for the dynamically screened interaction is exact $\stackrel{111}{=}$ This is a drastic simplification, so that the ease in which an arbitrary strong interaction in the clean case can be incorporated via the fully bosonized method is also the property of the functionalbosonization approach. In the disordered case, the functional-bosonization method has a technical advantage, especially useful for weak interactions, in that the conductivity is represented (in contrast to the fully bosonized approach) as a fermionic loop, albeit dressed by interactions in all possible ways.

\section{A. Functional bosonization: Background}

Our purpose here is to develop the functionalbosonization scheme in the presence of disorder and to apply it for the calculation of the weak-localization term (6.13) in the conductivity. The quantity to begin with is the fermionic Green's function. In the absence of disor- der, we write it in the Matsubara representation for an electron moving to the right $(+)$ or to the left $(-)$ as

$$
g_{\mu}(x, \tau)=g_{\mu}^{(0)}(x, \tau) e^{-B_{\mu \mu}(x, \tau)}, \quad \mu= \pm,
$$

where

$$
g_{ \pm}^{(0)}(x, \tau)=\mp \frac{i T}{2 v_{F}} \frac{1}{\sinh \left[\pi T\left(x / v_{F} \pm i \tau\right)\right]}
$$

is the free Green's function and the factor $\exp \left(-B_{\mu \mu}\right)$ accounts for interactions between electrons. The function $B_{\mu \mu}$ can be found by using the fact that, in the absence of backscattering due to e-e interaction, the Hubbard-Stratonovich transformation characterized by a decoupling field $\varphi(x, \tau)$ results in an identical representation of $g_{\mu}(x, \tau)$ as the average over $\varphi(x, \tau)$ of a function $\tilde{g}_{\mu}(x, \tau), 90,92,93$

$$
g_{\mu}(x, \tau)=\left\langle\tilde{g}_{\mu}(x, \tau)\right\rangle,
$$

where $\tilde{g}_{\mu}(x, \tau)$ describes the propagation of a particle of the same chirality in the field $\varphi(x, \tau)$ :

$$
\left[\partial_{\tau}-i \mu v_{F} \partial_{x}-i \varphi(x, \tau)\right] \tilde{g}_{\mu}(x, \tau)=\delta(x) \delta(\tau) .
$$

The angular brackets in Eq. (7.3) and throughout Sec. VII below denote the functional averaging over the field $\varphi(x, \tau)$. The representation (7.3) is equivalent to a local gauge transformation for the fermionic field of the right-/left-mover ${ }^{93}$

$$
\psi_{\mu}(x, \tau) \rightarrow \psi_{\mu}(x, \tau) \exp \left[i \theta_{\mu}(x, \tau)\right],
$$

where the bosonic field $\theta_{\mu}(x, \tau)$ is related to the decoupling field $\varphi(x, \tau)$ by

$$
\left(\partial_{\tau}-i \mu v_{F} \partial_{x}\right) \theta_{\mu}(x, \tau)=\varphi(x, \tau) .
$$

The Gaussian character of the Luttinger-liquid theory in the clean case means that the averaging in Eq. (7.3) is performed with an action in which all terms containing powers of $\varphi(x, \tau)$ higher than second vanish to zero. The correlator $\langle\varphi(x, \tau) \varphi(0,0)\rangle=V(x, \tau)$ then gives the dynamically screened RPA interaction.

As a result, the functions $B_{\mu \mu}(x, \tau)$ are represented as propagators of the fields $\theta_{\mu}(x, \tau)$ :

$$
B_{\mu \nu}(x, \tau)=\left\langle\left[\theta_{\mu}(0,0)-\theta_{\mu}(x, \tau)\right] \theta_{\nu}(0,0)\right\rangle,
$$

where for later use we also introduce the correlator of fields $\theta_{\mu}$ of different chirality, $\mu, \nu= \pm$. It is convenient to put the bare coupling constant $g_{4}=0$, shift the Fermi velocity accordingly (as discussed in Sec with two different interaction propagators $V_{++} \neq V_{+-}$ (see Appendix A). Then the correlators $B_{\mu \nu}(x, \tau)$ read

$$
\begin{aligned}
& B_{+ \pm}(x, \tau)=T \sum_{n} \int \frac{d q}{2 \pi}\left(e^{i q x-i \Omega_{n} \tau}-1\right) \\
& \times \frac{V_{+ \pm}\left(i \Omega_{n}, q\right)}{\left(i \Omega_{n}-q v_{F}\right)\left(i \Omega_{n} \mp q v_{F}\right)},
\end{aligned}
$$


where $\Omega_{n}=2 \pi n T$ is the bosonic frequency, $B_{--}(x, \tau)=$ $B_{++}(-x, \tau)$, and $B_{-+}(x, \tau)=B_{+-}(x, \tau)$. The integration with $V_{\mu \nu}$ given by Eq. (A.5) [or, equivalently, by Eqs. A.7, (A.8) with a renormalized Fermi velocity, see discussion in Sec. IIIA yields (see Appendix D):

$$
\begin{aligned}
B_{\mu \mu}(x, \tau) & =-\ln \eta_{\mu}(x, \tau)-\frac{\alpha_{b}}{2} \mathcal{L}(x, \tau), \\
B_{\mu,-\mu}(x, \tau) & =-\frac{\alpha_{r}}{2} \mathcal{L}(x, \tau),
\end{aligned}
$$

where

$$
\alpha_{b}=(1-K)^{2} / 2 K, \quad \alpha_{r}=\left(1-K^{2}\right) / 2 K,
$$

and

$$
\mathcal{L}(x, \tau)=\ln \frac{(\pi T / \Lambda)^{2}}{\sinh [\pi T(x / u+i \tau)] \sinh [\pi T(x / u-i \tau)]}
$$

The functions

$$
\eta_{ \pm}(x, \tau)=\frac{v_{F}}{u} \frac{\sinh \left[\pi T\left(x / v_{F} \pm i \tau\right)\right]}{\sinh [\pi T(x / u \pm i \tau)]}
$$

replace $v_{F} \rightarrow u$ in the free Green's functions $g_{ \pm}^{(0)}(x, \tau)$ in Eq. (7.1).

\section{B. Functional bosonization: Diagrammatic technique}

The exactness of the RPA approximation in 1d suggests the following diagrammatic technique. The Green's function (7.1) for a right-mover propagating from point $1=\left(x_{1}, \tau_{1}\right)$ to point $2=\left(x_{2}, \tau_{2}\right)$ can be represented by the diagram in Fig. 13. where the solid line is $g_{+}^{(0)}\left(x_{2}-x_{1}, \tau_{2}-\tau_{1}\right)$ and the wavy line connecting the end points denotes the pairing of two fluctuating factors $\exp \left[i \theta_{+}(2)\right]$ and $\exp \left[-i \theta_{+}(1)\right]$ with the Gaussian fields $\theta_{+}(2)$ and $\theta_{+}(1)$ :

$$
\begin{aligned}
& \left\langle\exp \left\{i\left[\theta_{+}(2)-\theta_{+}(1)\right]\right\}\right\rangle \\
& =\exp \left[-B_{++}\left(x_{2}-x_{1}, \tau_{2}-\tau_{1}\right)\right] .
\end{aligned}
$$

The fields $\theta_{+}(2)$ and $\theta_{+}(1)$ enter the exponent of Eq. (7.14) with different signs, according to the direction of the arrow on the solid line.

Consider now a backscattering vertex which describes reflection of the right-mover from an impurity (Figs. 14 and 15. before and after averaging over the fluctuations of $\theta_{\mu}$, respectively). The Green's function propagating from point 1 to point 3 upon backscattering on the static potential $U_{b}(x)$ at point 2 is given by

$$
\begin{aligned}
& \delta g\left(x_{3}, x_{1}, \tau_{3}-\tau_{1}\right)=\int_{0}^{1 / T} d \tau_{2} \int d x_{2} U_{b}\left(x_{2}\right) \\
\times & g_{+}^{(0)}\left(x_{2}-x_{1}, \tau_{2}-\tau_{1}\right) g_{-}^{(0)}\left(x_{3}-x_{2}, \tau_{3}-\tau_{2}\right) \\
\times & \left\langle\exp \left\{i\left[\theta_{+}(2)-\theta_{+}(1)+\theta_{-}(3)-\theta_{-}(2)\right]\right\}\right\rangle .
\end{aligned}
$$
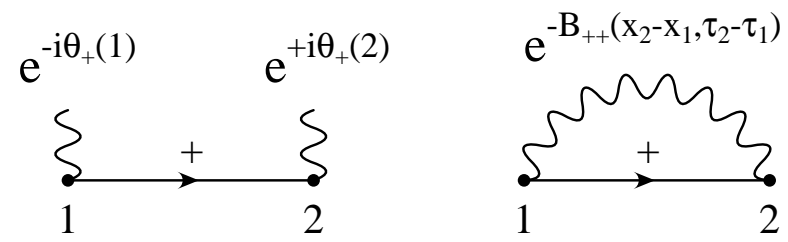

FIG. 13: (a) The Green's function of a right-mover propagating between points $1=\left(x_{1}, \tau_{1}\right)$ and $2=\left(x_{2}, \tau_{2}\right)$ in a given field $\varphi(x, \tau)$ acquires two phase factors $\exp \left[i \theta_{+}(2)\right]$ and $\exp \left[-i \theta_{+}(1)\right]$ (denoted as wavy lines) at the end points. Solid line: the free Green's function $g_{+}^{(0)}\left(x_{2}-x_{1}, \tau_{2}-\tau_{1}\right)$. (b) The wavy line connecting the points 1 and 2 denotes averaging of the phase factors over fluctuations of $\theta_{+}(1)$ and $\theta_{+}(2)$.

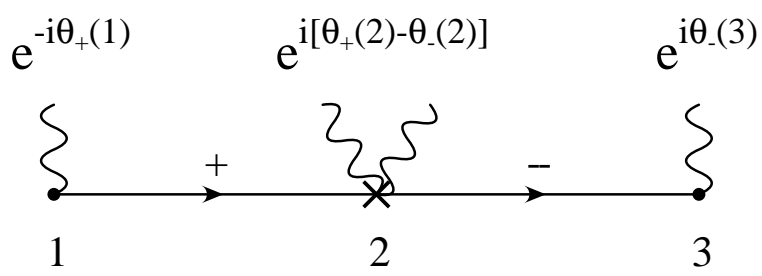

FIG. 14: The Green's function with a backscattering vertex (marked by a cross) between the end points. The vertex is dressed by a local fluctuating factor $\exp \left\{i\left[\theta_{+}(2)-\theta_{-}(2)\right]\right\}$.

A direct consequence of Eq. (7.5) is that the vertex at point 2 is dressed by the local fluctuating factor $\exp \left\{i\left[\theta_{+}(2)-\theta_{-}(2)\right]\right\}$. The correlations of the fields $\theta_{\mu}$ at different points in $x$ space in the last factor of Eq. (7.15) account for e-e interaction. Apart from the averages (7.14) of the fields of the same chirality, there appear averages of the type

$$
\begin{aligned}
& \left\langle\exp \left\{i\left[\theta_{+}(2)-\theta_{-}(1)\right]\right\}\right\rangle \\
& =\exp \left[-B_{+-}\left(x_{2}-x_{1}, \tau_{2}-\tau_{1}\right)-M_{0}\right]
\end{aligned}
$$

where

$$
M_{0}=\left\langle\theta_{+}^{2}(0,0)\right\rangle-\left\langle\theta_{+}(0,0) \theta_{-}(0,0)\right\rangle .
$$

Note that the factors $\exp \left( \pm M_{0}\right)$ cancel out in any closed fermionic loop, so that in the diagrammatic technique for observables one can omit them from the very beginning. The Gaussian character of the correlations means that the exponent of the last factor in Eq. (7.15) is represented as a sum of the correlators $B_{\mu \nu}$ :

$$
\begin{aligned}
& \left\langle\exp \left\{i\left[\theta_{+}(2)-\theta_{+}(1)+\theta_{-}(3)-\theta_{-}(2)\right]\right\}\right\rangle \\
& =\exp \left[-B_{++}\left(x_{2}-x_{1}, \tau_{2}-\tau_{1}\right)\right. \\
& +B_{+-}\left(x_{2}-x_{1}, \tau_{2}-\tau_{1}\right)-B_{--}\left(x_{3}-x_{2}, \tau_{3}-\tau_{2}\right) \\
& \left.+B_{+-}\left(x_{3}-x_{2}, \tau_{3}-\tau_{2}\right)-B_{+-}\left(x_{3}-x_{1}, \tau_{3}-\tau_{1}\right)\right],
\end{aligned}
$$

as illustrated in Fig. 15] [we used in Eq. (7.18) the fact that $\left.B_{+-}(2,2)=0\right]$. 


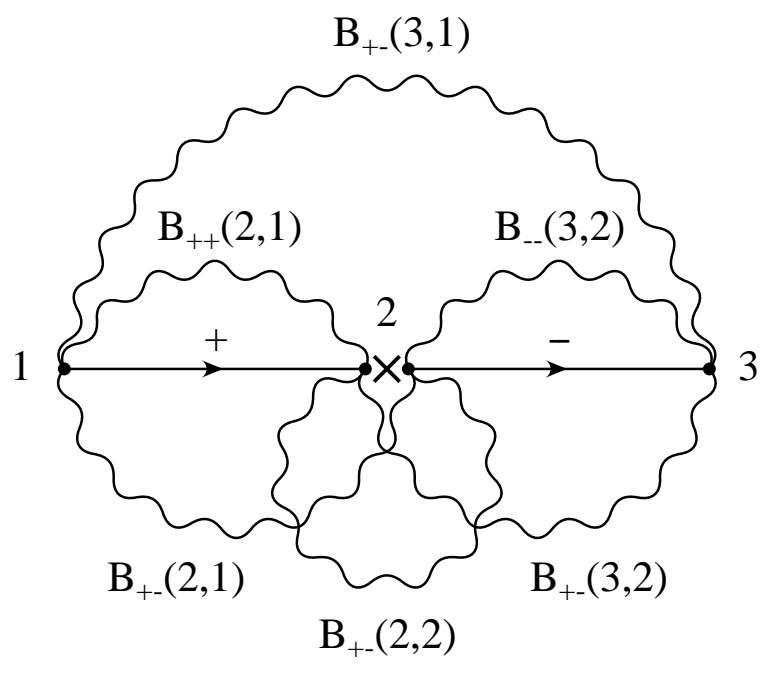

FIG. 15: Illustration of the diagrammatic technique with the propagators $B_{\mu \nu}$ : the correction $\delta g\left(x_{3}, x_{1}, \tau_{3}-\tau_{1}\right)$ [Eq. (7.15)] to the Green's function due to the backscattering at point 2 . The wavy lines denote averaging of the fluctuating factors $\exp \left( \pm \theta_{\mu}\right)$ shown in Fig. 14. The pairing of all the fields $\theta_{\mu}$ with each other according to Eq. (7.7) yields the corresponding contributions $B_{\mu \nu}$ to the factor (7.18). For ease of visualization, the ends of the free Green's functions are split off from the backscattering vertex at point 2 .

The appearance of the combination $\theta_{+}(2)-\theta_{-}(2)$ for backscattering at point 2 in Eq. (17.15) and the factorization of the correlations (7.18) in the simplest case of a single backscattering vertex suggests a way to proceed to two or more vertices. In effect, the e-e interaction can be completely gauged out to the impurity vertices, as emphasized in Ref. 93. Specifically, one can, in principle, calculate observables (given by closed fermionic loops) perturbatively to any order in the strength of disorder and exactly in the strength of e-e interaction by attaching a factor

$$
\exp \left\{ \pm i\left[\theta_{+}(N)-\theta_{-}(N)\right]\right\}
$$

to each backscattering vertex, where $N$ labels the vertex, and pairing all the fields $\theta_{\mu}(N)$ with each other. The averaging over the fluctuations of $\theta_{\mu}(N)$ is then performed with the correlators (7.9) - (7.10) calculated for a homogeneous system. The sign \pm in Eq. (7.19) should be chosen to correspond to the chirality with which an electron is incident on the impurity.

The WL correction to the conductivity is given by the fermionic loop with 6 backscattering vertices (Fig. 5), each of which should be dressed with the factors (7.19). However, taking disorder into account at this level would not be sufficient. As we showed in Sec.VI in order to calculate the effect of dephasing for spinless electrons, disorder should also be included, to first order in the strength of disorder $\gamma$, in the loops that make up the RPA series for the effective interaction. We recall that in the RPA approximation, substantiated in Sec. VI, no more than two external interaction lines connect to each of the fermionic loops that constitute the interaction propagator and the averaging of the interaction propagator over disorder is performed in such a way that no impurity lines connect different fermionic loops. As we show in Appendix E, in the language of the functional bosonization, the RPA approximation in the presence of disorder translates into a disorder-induced renormalization of the correlators $B_{\mu \nu}(x, \tau)$. Specifically, the renormalization consists in adding a disorder-induced damping to the propagators $V_{++}$and $V_{+-}$in Eq. (7.8). The renormalized interaction propagators are derived in Appendix A [see Eqs. A.24 - A.25] and are given in the Matsubara representation by

$$
\begin{aligned}
& V_{++}\left(i \Omega_{n}, q\right)=-\frac{g_{2}^{2}}{2 \pi v_{F}} \frac{q^{2} v_{F}^{2}-i q v_{F} \Omega_{n}+\left|\Omega_{n}\right| \gamma / 2}{q^{2} u^{2}+\Omega_{n}^{2}+\left|\Omega_{n}\right|(\gamma+\delta \gamma)}, \\
& V_{+-}\left(i \Omega_{n}, q\right)=g_{2} \frac{q^{2} v_{F}^{2}+\Omega_{n}^{2}+\left|\Omega_{n}\right|(\gamma+\delta \gamma / 2)}{q^{2} u^{2}+\Omega_{n}^{2}+\left|\Omega_{n}\right|(\gamma+\delta \gamma)},
\end{aligned}
$$

where $\gamma$ denotes the transport scattering rate, Eq. (A.13), and $\delta \gamma$ is given by Eq. A.26 (at variance with the preceding part of the paper, in this section we do not use the notation $\tau$ for the transport scattering time in order to avoid a possible confusion with the Matsubara time).

Thus, our strategy in deriving Eq. 6.13) via the functional bosonization is this: find the disorder-renormalized correlators $B_{\mu \nu}(x, \tau)$ to order $\mathcal{O}\left(\gamma g_{2}^{2}\right)$ and use them to average over fluctuations of the fields $\theta_{\mu}$ "attached" to 6 impurity vertices of the 3-impurity Cooperon (Fig. 16). The diagrams (b) and (c) in Fig. 5] are calculated in the same way.

\section{Functional bosonization with a disorder-renormalized RPA}

We now turn to the calculation of the effective (disorder-renormalized) propagators $B_{\mu \nu}(x, \tau)$ to order $\mathcal{O}\left(\gamma g_{2}^{2}\right)$ by using Eq. (7.8) and the interaction propagators $V_{++}$and $V_{+-}$given by Eqs. (7.20), (7.21). At second order in interaction, $B_{++}(x, \tau)$ reads

$$
\begin{aligned}
& B_{++}(x, \tau) \simeq-\frac{g_{2}^{2}}{2 \pi v_{F}} T \sum_{n} \int \frac{d q}{2 \pi}\left(e^{i q x-i \Omega_{n} \tau}-1\right) \\
& \times \frac{q^{2} v_{F}^{2}-i q v_{F} \Omega_{n}+\left|\Omega_{n}\right| \gamma / 2}{\left(-i \Omega_{n}+q v_{F}\right)^{2}\left(q^{2} v_{F}^{2}+\Omega_{n}^{2}+\left|\Omega_{n}\right| \gamma\right)} .
\end{aligned}
$$

Let us represent $B_{++}$as a sum $B_{++}=\mathcal{B}_{0}+\mathcal{B}_{>}+\mathcal{B}_{<}$, where $\mathcal{B}_{0}(x)$ is the contribution of $\Omega_{n}=0, \mathcal{B}_{>}(x, \tau)$ and $\mathcal{B}_{<}(x, \tau)=\mathcal{B}_{>}(-x,-\tau)$ are the contributions of $\Omega_{n}>0$ and $\Omega_{n}<0$, respectively. In turn, $\mathcal{B}_{>}(x, \tau)$ is a sum of two terms:

$$
\mathcal{B}_{>}=\mathcal{B}_{>}^{d p}+\mathcal{B}_{>}^{s p}
$$




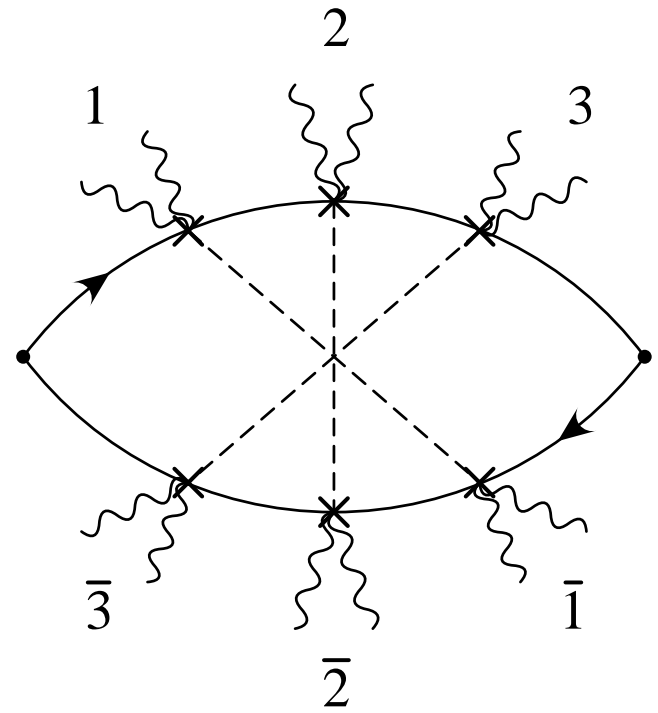

FIG. 16: The three-impurity Cooperon diagram with interaction effects encoded in the fluctuating factors $\exp \left( \pm \theta_{\mu}\right)$ (denoted by the wavy lines) attached to the backscattering vertices. Each impurity vertex $N=\left(x_{N}, \tau_{N}\right)$ is characterized by two fluctuating fields $\theta_{ \pm}(N)$, and similarly for $\bar{N}=\left(x_{N}, \bar{\tau}_{N}\right)$. Pairing of all the fields with each other (similarly to Figs. 14[15) describes both the elastic renormalization of the three impurities and the dephasing of the Cooperon.

where $\mathcal{B}_{>}^{d p}$ is the contribution of the double pole at $q v_{F}=$ $i \Omega_{n}$ and $\mathcal{B}_{>}^{s p}$ is the contribution of the single poles at $q v_{F}= \pm i\left(\Omega_{n}^{2}+\Omega_{n} \gamma\right)^{1 / 2}$.

The term coming from zero frequency does not depend on $\gamma$ :

$$
\mathcal{B}_{0}(x)=-\frac{g_{2}^{2}}{2 \pi v_{F}} T \int \frac{d q}{2 \pi} \frac{e^{i q x}-1}{q^{2} v_{F}^{2}}=\alpha^{2} \frac{\pi T|x|}{v_{F}},
$$

where $\alpha=g_{2} / 2 \pi v_{F}$, as before. The contribution of the double pole does not depend on $\gamma$ either,

$$
\begin{aligned}
\mathcal{B}_{>}^{d p}(x, \tau) & =\alpha^{2} \frac{\pi T x}{v_{F}} \Theta(x) \\
& \times \frac{1}{\exp \left[2 \pi T\left(x / v_{F}+i \tau\right)\right]-1},
\end{aligned}
$$

and is an expansion of $\ln \eta_{+}(7.13)$ in powers of $\alpha$. Expanding the single-pole contribution to first order in $\gamma$, we have

$$
\begin{aligned}
\mathcal{B}_{>}^{s p}(x, \tau) & \simeq-\frac{\alpha^{2}}{4}\left\{\ln \frac{2 \pi T / \Lambda}{1-\exp \left[-2 \pi T\left(|x| / v_{F}+i \tau\right)\right]}\right. \\
& -\frac{\gamma|x|}{2 v_{F}} \ln \frac{1}{1-\exp \left[-2 \pi T\left(|x| / v_{F}+i \tau\right)\right]} \\
& \left.-\frac{\gamma}{4 \pi T} F\left[2 \pi T\left(\frac{|x|}{v_{F}}+i \tau\right)\right]\right\},
\end{aligned}
$$

where

$$
F(z)=\sum_{n=1}^{\infty} \frac{\exp (-n z)-1}{n^{2}}
$$

The sum (7.27) is defined for $\operatorname{Re} z>0$. At small $|z| \ll 1$, it behaves as $F(z) \simeq z \ln |z|$, which cancels the second term in Eq. (17.26) at the ultraviolet cutoff $\tau \rightarrow 0,|x| \rightarrow$ $1 / v_{F} \Lambda$. Below, we will need the analytical continuation of $B_{\mu \nu}(x, \tau)$ in the complex plane of $\tau$. In particular, an important contribution to the dephasing will come from the analytical continuation of $F(z)$ to large negative $\operatorname{Re} z<0$. The latter can be done, e.g., by means of the integral representation

$$
F(z)=\int_{0}^{z} d z^{\prime} \ln \left[1-\exp \left(-z^{\prime}\right)\right] .
$$

Piecing all the terms together, $B_{++}(x, \tau)$ with a disorder-induced correction of order $\mathcal{O}\left(\gamma g_{2}^{2}\right)$ is written as

$$
B_{++}=\left.B_{++}\right|_{\gamma=0}+b,
$$

where $\left.B_{++}\right|_{\gamma=0}$ is given by Eq. (7.9),,$b=b_{1}+b_{2}$, and

$$
\begin{aligned}
& b_{1}(x, \tau) \simeq \alpha^{2} \frac{\gamma}{16 \pi T}\left\{\left(\frac{2 \pi T x}{v_{F}}\right)^{2}+\frac{2 \pi T|x|}{v_{F}}\right. \\
&\left.\times \ln \frac{1}{4 \sinh \left[\pi T\left(x / v_{F}+i \tau\right)\right] \sinh \left[\pi T\left(x / v_{F}-i \tau\right)\right]}\right\}, \\
& b_{2}(x, \tau) \simeq \alpha^{2} \frac{\gamma}{16 \pi T}\left\{F\left[2 \pi T\left(\frac{|x|}{v_{F}}+i \tau\right)\right]\right. \\
&\left.+F\left[2 \pi T\left(\frac{|x|}{v_{F}}-i \tau\right)\right]\right\} .
\end{aligned}
$$

Note that the second term in Eq. (7.30) can be viewed as an $x$ dependent renormalization of $\alpha$ induced by disorder, $\delta \alpha=-\alpha \gamma|x| / 4 v_{F}$, as follows from a comparison with Eq. (7.9). This is consistent with the RG flow of the interaction constant (4.28). However, Eqs. (7.30), (7.30) contain more information and allow us to study the dephasing missed in the RG equations.

Consider now the disorder-renormalized propagator $B_{+-}(x, \tau)$. To second order in $g_{2}$,

$$
\begin{aligned}
& B_{+-}(x, \tau) \simeq-T \sum_{n} \int \frac{d q}{2 \pi} \frac{e^{i q x-i \Omega_{n} \tau}-1}{\Omega_{n}^{2}+q^{2} v_{F}^{2}} \\
& \times\left(g_{2}+\frac{g_{2}^{2}}{4 \pi v_{F}} \frac{\left|\Omega_{n}\right| \gamma}{q^{2} v_{F}^{2}+\Omega_{n}^{2}+\left|\Omega_{n}\right| \gamma}\right) .
\end{aligned}
$$

Expanding Eq. (7.31) to linear order in $\gamma$, we readily obtain a correction to $B_{+-}(x, \tau)$ [Eq. (17.10)] which is again given by Eqs. (17.30), (7.30) but with the opposite sign, so that finally

$$
B_{+ \pm}=\left.B_{+ \pm}\right|_{\gamma=0} \pm b .
$$

\section{Cooperon in a dynamic environment}

Now that we have found the propagators $B_{\mu \nu}(x, \tau)$, let us turn to the calculation of the WL correction to the conductivity. The interaction induces the factor

$$
\exp \left(-S_{C}\right)=\left\langle\exp \left[i\left(\theta_{f}-\theta_{b}\right)\right]\right\rangle
$$


to the Cooperon loop, where $\theta_{f}$ and $\theta_{b}$ are the phases accumulated by an electron propagating along the "forward" and "backward" paths (Fig. 17 represents the paths from Fig. 6a in a way which is more convenient here) and the averaging is performed with the disorderrenormalized correlators (7.32). Again, $S_{C}$ accounts for both the dephasing and the elastic renormalization of impurities. The factor (7.33) is the same for all 3 diagrams for $\Delta \sigma_{\mathrm{wl}}$ in Fig. 5 .

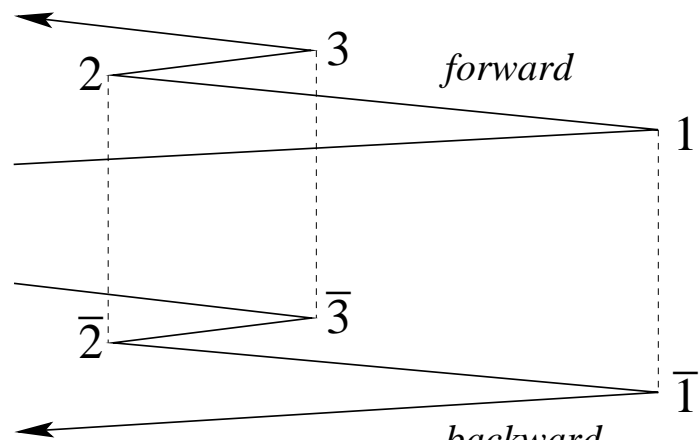

FIG. 17: Two time-reversed paths whose interference constitutes the three-impurity Cooperon. There are three backscatterings off impurities on each of the paths (the positions of the impurities are marked by the dashed lines). The onedimensional paths are stretched vertically for ease of visualization, with the "time axis" directed upwards for the forward path and downwards for the backward path. Note that this representation of the paths is identical to the graphs in the Berezinskii technique. ${ }^{69}$

The phases $\theta_{f, b}$ are given by

$$
\begin{aligned}
& \theta_{f}=\sum_{N=1}^{3}(-1)^{N+1}\left[\theta_{+}(N)-\theta_{-}(N)\right], \\
& \theta_{b}=\sum_{\bar{N}=1}^{3}(-1)^{\bar{N}+1}\left[\theta_{+}(\bar{N})-\theta_{-}(\bar{N})\right],
\end{aligned}
$$

where $N, \bar{N}$ are the impurity-vertex numbers, for the forward and backward paths, respectively; as shown in Fig. 6. The exponent in Eq. (7.33) is represented as

$$
S_{C}=-2\left(M_{f f}-M_{f b}\right)
$$

where

$$
\begin{aligned}
M_{f f} & =-\frac{1}{2}\left\langle\theta_{f}^{2}\right\rangle \\
& =\frac{1}{2} \sum_{N N^{\prime}}(-1)^{N+N^{\prime}} M\left(N, N^{\prime}\right)-M_{0}, \\
M_{f b} & =-\frac{1}{2}\left\langle\theta_{f} \theta_{b}\right\rangle \\
& =\frac{1}{2} \sum_{N \bar{N}}(-1)^{N+\bar{N}} M(N, \bar{N})-M_{0},
\end{aligned}
$$

and all the pairings between the phases associated with impurities $N$ and $N^{\prime}$ are given by the combination

$$
M\left(N, N^{\prime}\right)=B_{++}\left(x_{N}-x_{N^{\prime}}, \tau_{N}-\tau_{N^{\prime}}\right)
$$

$$
\begin{aligned}
& +B_{--}\left(x_{N}-x_{N^{\prime}}, \tau_{N}-\tau_{N^{\prime}}\right) \\
& -2 B_{+-}\left(x_{N}-x_{N^{\prime}}, \tau_{N}-\tau_{N^{\prime}}\right)
\end{aligned}
$$

and similarly for the pairings between $N$ and $\bar{N}$. The constant $M_{0}$ given by Eq. (7.17) cancels out in $S_{C}$. Note that on the closed contour $\left\langle\theta_{f}^{2}\right\rangle=\left\langle\theta_{b}^{2}\right\rangle$, which is accounted for by the factor of 2 in Eq. (7.36).

\section{E. Diffuson ladder and renormalization of disorder}

Before calculating the three-impurity Cooperon, it is instructive to look at the diffuson diagrams. More specifically, let us consider first the ladder diagrams without including self-energy corrections.

\section{One impurity line}

The simplest diagram for the density-density correlator of order $\mathcal{O}(\gamma)$ is shown in Fig. 18 and written as

$$
\begin{aligned}
d_{1} & =\frac{v_{F} \gamma_{0}}{2} \int_{0}^{1 / T} d \tau_{1} \int_{0}^{1 / T} d \bar{\tau}_{1} \\
& \times g_{+}^{(0)}\left(x_{1}, \tau_{1}\right) g_{-}^{(0)}\left(x-x_{1}, \tau-\tau_{1}\right) \exp [-M(1, \overline{1})] \\
& \times g_{+}^{(0)}\left(-x_{1},-\bar{\tau}_{1}\right) g_{-}^{(0)}\left(x_{1}-x, \bar{\tau}_{1}-\tau\right)
\end{aligned}
$$

The dashed line gives the bare strength of backscattering $v_{F} \gamma_{0} / 2$. In Eq. (7.40) we only integrate over internal time variables, keeping other coordinates in $(x, \tau)$ space fixed. The factor $\exp [-M(1, \overline{1})]$, which is taken at the same spatial point, depends on $\tau_{1}-\bar{\tau}_{1}$ only:

$$
\begin{gathered}
\exp [-M(1, \overline{1})]=\left\{\left(\frac{\Lambda}{\pi T}\right)^{2} \sin \left[\pi T\left(\tau_{1}-\bar{\tau}_{1}+\frac{i}{\Lambda}\right)\right]\right. \\
\left.\times \sin \left[\pi T\left(\tau_{1}-\bar{\tau}_{1}-\frac{i}{\Lambda}\right)\right]\right\}^{\alpha_{e}}
\end{gathered}
$$

where we used the ultraviolet cutoff from Eq. (D.7) and

$$
\alpha_{e}=\alpha_{r}-\alpha_{b}=1-K
$$

Note that the interaction-induced phase factors cancel out at the external vertices which do not change chirality. In particular, this means that in Eq. (7.40) there is no renormalization of the velocity by the factors (7.13). The latter is a general property of fermionic loops with a larger number of impurities: the Green's functions connecting to the external vertices with a small momentum transfer are characterized by the bare Fermi velocity $v_{F}$.

In the absence of e-e interaction, $d_{1}$ is equal to

$$
d_{1}^{(0)}=\frac{v_{F} \gamma_{0}}{2} I^{2}
$$




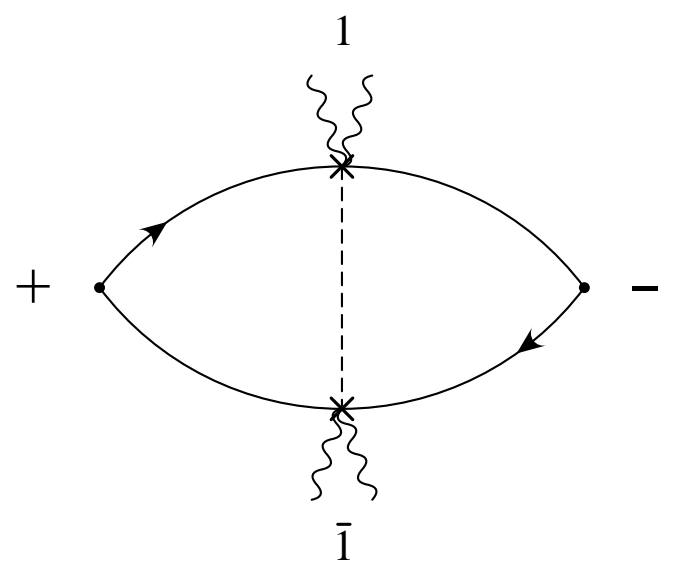

FIG. 18: Density-density correlator of first order in the impurity strength. The backscattering vertices are marked by the crosses. Pairing of the fluctuating factors $\exp \left( \pm \theta_{\mu}\right)$ (denoted by the wavy lines) with each other yields the factor $\exp [-M(1, \overline{1})]$ in Eq. (7.40), which describes the renormalization of the impurity strength by the interaction.

where

$$
\begin{aligned}
& I=\int_{0}^{1 / T} d \tau_{1} g_{+}^{(0)}\left(x_{1}, \tau_{1}\right) g_{-}^{(0)}\left(x-x_{1}, \tau-\tau_{1}\right) \\
& =-\frac{T}{2 v_{F}^{2}} \frac{1}{\sinh \left\{\pi T\left[\left(2 x_{1}-x\right) / v_{F}+i \tau\right]\right\}} \\
& \times\left[\Theta\left(x_{1}\right) \Theta\left(x_{1}-x\right)-\Theta\left(-x_{1}\right) \Theta\left(x-x_{1}\right)\right] .
\end{aligned}
$$

What is important to us here is that $I$ is given by the pole on the classical trajectory $t_{1}=x_{1} / v_{F}$ of a rightmoving electron (for $x_{1}>0$ ) or a left-moving hole (for $x_{1}<0$ ), where $t_{1}=-i \tau_{1}$ (Fig. 19). If we proceed to the $\Omega$ representation, the diagram for nonzero $x$ and $x_{1}$ is written as

$$
\begin{aligned}
& \int_{0}^{1 / T} d \tau e^{i \Omega_{n} \tau} d_{1}^{(0)}=\frac{\gamma_{0}}{4 \pi v_{F}^{3}}\left|\Omega_{n}\right| e^{-\left|\Omega_{n}\left(2 x_{1}-x\right)\right|} \\
& \times\left[\Theta\left(x_{1}\right) \Theta\left(x_{1}-x\right) \Theta\left(\Omega_{n}\right)+\Theta\left(-x_{1}\right) \Theta\left(x-x_{1}\right) \Theta\left(-\Omega_{n}\right)\right]
\end{aligned}
$$

and again is given by the (double) pole on the classical trajectory $t=\left(2 x_{1}-x\right) / v_{F}$, where $t=-i \tau$ and $\left|2 x_{1}-x\right|$ is the total length of the trajectory from point $x=0$ to point $x$ with backscattering at point $x_{1}$. This simple consideration suggests that the two-particle correlators in the presence of a weak e-e interaction should still be determined by a close vicinity of the classical trajectory.

To evaluate the integrals (7.40) at $\alpha_{e} \neq 0$, we assume for definiteness that $x_{1}>0$ and $x<x_{1}$. The singularities of the integrand of Eq. (7.40) in the upper half-plane of $\bar{\tau}_{1}$ for $-0<\operatorname{Re} \bar{\tau}_{1}<1 / T-0$ are then a pole at $\bar{\tau}_{1}=i x_{1} / v_{F}$ and a branch cut which comes from the factor (7.41). The branch cut starts at $\bar{\tau}_{1}=\tau_{1}+i / \Lambda$ and is sent upwards, as shown in Fig. 20a. The contour of integration over $\bar{\tau}_{1}$ can be closed upwards, so that the integral over $\bar{\tau}_{1}$ along the real axis from $\bar{\tau}_{1}=0$ to $\bar{\tau}_{1}=1 / T$ is given exactly

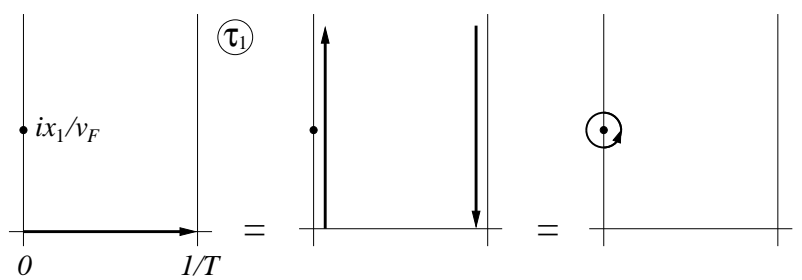

FIG. 19: Analytical structure of the integrand in Eq. (7.44), with a pole at $\tau_{1}=i x_{1} / v_{F}$, for $x_{1}>0$ and $x<x_{1}$. Closing the contour of integration upwards, the integral along the real axis of the Matsubara time is represented as a sum of two integrals along the imaginary axis at $\tau_{1}=+0$ and $\tau_{1}=1 / T-0$. Using the periodicity with respect to the shift $\tau_{1} \rightarrow \tau_{1} \pm 1 / T$, the sum gives a residue at the pole.

by the contributions of the pole and the branch cut. The contribution of the latter is proportional to $\alpha_{e}$ and can be omitted in the limit of weak interaction, after which the remaining integral over $\tau_{1}$ reads

$$
\begin{aligned}
d_{1} & \simeq \frac{\gamma_{0}}{2}\left(\frac{T}{2 v_{F}}\right)^{3} \frac{(\Lambda / \pi T)^{2 \alpha_{e}}}{\sinh \left\{\pi T\left[\left(2 x_{1}-x\right) / v_{F}+i \tau\right]\right\}} \\
& \times \int_{C} d \tau_{1} \frac{\sin ^{-1+2 \alpha_{e}}\left[\pi T\left(\tau_{1}-i x_{1} / v_{F}\right)\right]}{\sin \left\{\pi T\left[\tau_{1}-\tau+i\left(x_{1}-x\right) / v_{F}\right]\right\}}
\end{aligned}
$$

Here the contour of integration $C$ in the complex plane of $\tau_{1}$ runs anticlockwise around the branch cut along the imaginary axis of $\tau_{1}$, Fig. 20 p. For $\left|\alpha_{e}\right| \ll 1$ the singularity in Eq. (7.45) is almost a pole on the classical trajectory $t_{1}=x_{1} / v_{F}$ with $t_{1}=-i \tau_{1}$, so that, indeed, the system dynamics is determined by a close vicinity of the classical trajectory. We observe, however, that taking the interaction-dependent factor in the integrand straight at $t_{1} \rightarrow x_{1} / v_{F}$ yields an ultraviolet singularity that exactly cancels the factor $(\Lambda / \pi T)^{2 \alpha_{e}}$ in front of the integral in Eq. (7.45), which returns us to the noninteracting result. It is thus the integration in a close vicinity $t_{1}-x_{1} / v_{F} \lesssim 1 / T$ of the classical trajectory along the branch cut that gives the renormalization of disorder by interaction. If $\left|\alpha_{e}\right| \ll 1$, Eq. (7.45) reduces to

$$
d_{1} \simeq d_{1}^{(0)}(\Lambda / \pi T)^{2 \alpha_{e}} .
$$

The difference between Eq. (7.46) and the noninteracting result consists solely in the last factor which describes the renormalization of the disorder strength $\gamma_{0} \rightarrow \gamma$, where

$$
\gamma=\gamma_{0}(\Lambda / \pi T)^{2 \alpha_{e}}
$$

\section{2. "Two-impurity diffuson"}

Before generalizing to the Cooperon diagram, it is useful to consider a diagram of order $\mathcal{O}\left(\gamma^{2}\right)$ with two parallel impurity lines, Fig. 21. What is new in this "twoimpurity diffuson" as compared to Eq. (7.40) is that now 


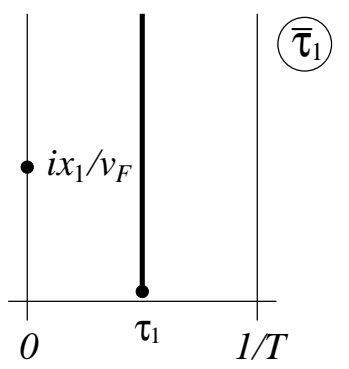

a)

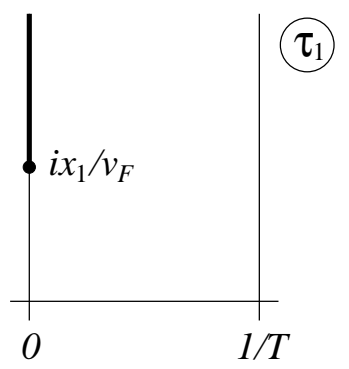

b)
FIG. 20: Analytical structure of the integrand in Eq. (7.40). (a) The contour of integration over $\bar{\tau}_{1}$ is closed upwards where the singularities of the integrand for $x_{1}>0$ and $x<x_{1}$ are a pole at $\bar{\tau}_{1}=i x_{1} / v_{F}$ and a branch cut which starts at $\bar{\tau}_{1}=\tau_{1}+i / \Lambda$ and runs upwards. (b) The integral over $\tau_{1}$ of the contribution of the pole in Fig. 20, is given exactly by the branch cut starting at $\tau_{1}=i x_{1} / v_{F}$ and running upwards along the imaginary axis of $\tau_{1}$.

there are factors $\exp ( \pm M)$ which connect different impurities. Specifically, the interaction-induced factor is

$$
\begin{aligned}
& \exp [-M(1, \overline{1})-M(2, \overline{2}) \\
& -M(1,2)-M(\overline{1}, \overline{2})+M(1, \overline{2})+M(\overline{1}, 2)] .
\end{aligned}
$$

Each of the cross-terms (the last 4 terms above), when taken on the classical trajectory, may give a large contribution to the exponent of Eq. (7.48). An important observation, however, is that on the classical trajectory they cancel each other exactly. This can be seen immediately since on the classical trajectory $\tau_{1}=\bar{\tau}_{1}$ and at this point $M(1,2)=M(\overline{1}, 2)$ and $M(\overline{1}, \overline{2})=M(1, \overline{2})$. The cancellation can be verified for diffuson diagrams of higher order in $\gamma$ and is a manifestation of the mere fact that for the density-density correlator there is no dephasing in the diffuson channel. No dephasing is generated both for the bare correlators $B_{\mu \nu}(x, \tau)$ and for those renormalized by disorder at the level of RPA.

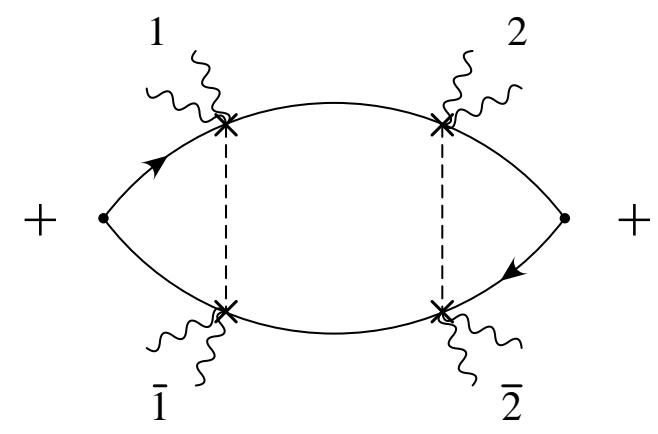

FIG. 21: "Two-impurity" diffuson with the fluctuating factors $\exp \left[ \pm \theta_{\mu}(N)\right]$ (denoted by the wavy lines) attached to the backscattering vertices (marked by the crosses).

As far as the integration around the classical trajectory of the two-impurity diffuson is concerned, it suffices for our purposes here to analyze the case of weak interaction. The analytical structure of the integral over four time variables, $\tau_{1}, \tau_{2}, \bar{\tau}_{1}$, and $\bar{\tau}_{2}$ can be simplified by selecting the singularities that are strongest in the limit $\left|\alpha_{e}\right| \ll 1$. Let us assume for definiteness that $x_{1}>0$ and $x_{2}<x_{1}$. In the plane of $\tau_{1}$ we have for $\operatorname{Im} \tau_{1} \geq 0$ : a pole at $\tau_{1}=$ $i x_{1} / v_{F}$ and three branch cuts starting at $\tau_{1}=\bar{\tau}_{1}+i / \Lambda$, $\tau_{2}+i\left(x_{1}-x_{2}\right) / u$, and $\bar{\tau}_{2}+i\left(x_{1}-x_{2}\right) / u$, as shown in Fig. 222. For weak interaction, these branch cuts are "weak" in the sense that they are characterized by a small exponent $\alpha_{e}$. The approximation we make consists in neglecting all weak cuts and keeping only "strong" cuts characterized by exponents close to 1 . Taking into account the weak cuts yields corrections small in $\alpha_{e} \ll 1$.

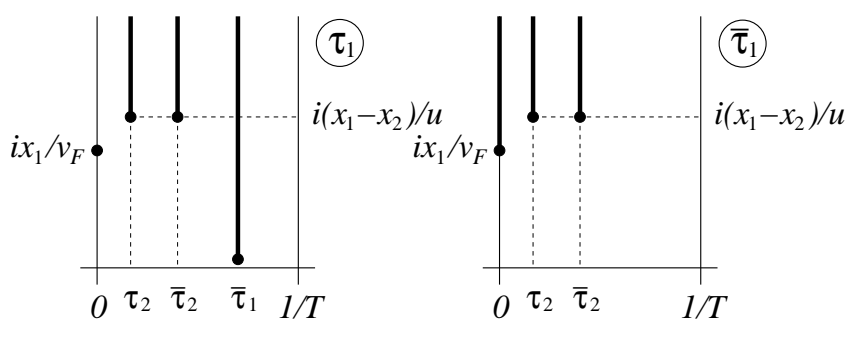

a)

b)

FIG. 22: Integration over the internal times in the twoimpurity diffuson shown in Fig. 21. (a) The analytical structure of the integral in the complex plane of $\tau_{1}$ : the contour of integration runs around a pole and three vertical branch cuts. (b) The contour of integration in the complex plane of $\bar{\tau}_{1}$ runs around three vertical branch cuts.

Picking up $\tau_{1}$ at the pole $\tau_{1}=i x_{1} / v_{F}$ yields the following singularities in the plane of $\bar{\tau}_{1}$ for $\operatorname{Im} \bar{\tau}_{1} \geq 0$ : a strong branch cut at $\bar{\tau}_{1}=i x_{1} / v_{F}$ and two weak branch cuts at $\bar{\tau}_{1}=\tau_{2}+i\left(x_{1}-x_{2}\right) / u$ and $\bar{\tau}_{2}+i\left(x_{1}-x_{2}\right) / u$. Neglecting the weak cuts and integrating along the strong one similarly to Eq. (7.45) gives the renormalization of the impurity at point $x_{1}$. During the integration along the strong cut, we pick up other factors in the integrand at the classical trajectory, i.e., at the starting point of the strong cut, which yields a cancellation of the cross-terms connecting two impurities. The remaining double integral over $\tau_{2}$ and $\bar{\tau}_{2}$ with the factor $\exp [-M(2, \overline{2})]$ has the same structure as in the case of a single impurity and gives the renormalization of the impurity at point $x_{2}$. The net result is that the two-impurity diffuson $d_{2}$ for $\left|\alpha_{e}\right| \ll 1$ simply acquires two identical factors $(\Lambda / \pi T)^{2 \alpha_{e}}$ which renormalize the impurities:

$$
d_{2} \simeq d_{2}^{(0)}(\Lambda / \pi T)^{4 \alpha_{e}}
$$

where

$$
\begin{aligned}
d_{2}^{(0)} & =\left(\frac{\gamma_{0}}{2 v_{F}}\right)^{2} \frac{\left(T / 2 v_{F}\right)^{2}}{\sinh ^{2}\left\{\pi T\left[\left(x+2 x_{1}-2 x_{2}\right) / v_{F}+i \tau\right]\right\}} \\
& \times\left[\Theta\left(x_{1}\right) \Theta\left(x_{1}-x_{2}\right) \Theta\left(x-x_{2}\right)\right. \\
& \left.+\Theta\left(-x_{1}\right) \Theta\left(x_{2}-x_{1}\right) \Theta\left(x_{2}-x\right)\right] .
\end{aligned}
$$


Self-energy corrections are incorporated in the same way leading to an additional factor $d_{2} \rightarrow d_{2} \exp \left(-\gamma \mid x+2 x_{1}-\right.$ $2 x_{2} \mid / v_{F}$ ), where $\gamma$ is given by Eq. (7.47). This procedure is straightforwardly extended to the diffuson ladder with three or more impurity legs, so that the difference of the full diffuson ladder in the presence of e-e interaction from that in the absence of interaction is only in the change $\gamma_{0} \rightarrow \gamma$ [Eq. (7.47)].

\section{F. Dephasing action and weak-localization correction}

Let us now return to the three-impurity Cooperon whose interaction-induced factor is given by Eqs. (7.33),(7.36)-(7.39). As the analysis of the two-impurity diffuson demonstrated, the analytical structure of the diagrams with increasing number of factors $\exp ( \pm M)$ rapidly gets quite complicated. The same example suggested, however, a systematic way to deal with the limit of weak interaction by ignoring in the first approximation all weak branch cuts. The integration around strong cuts then leads to the renormalization of impurities, while picking up nonsingular factors at the classical trajectory (the starting points of the strong cuts) generates the dephasing action. At this level, the calculation of the Cooperon is similar to that of the diffuson, with one essential difference: in contrast to the diffuson, the Cooperon factor $\exp \left(-S_{C}\right)$, Eq. (7.33), does not vanish on the classical trajectory and hence leads to dephasing.

Another important issue is related to the definition of the classical trajectory for the Cooperon; more specifically, to the question of what is the velocity that characterizes this trajectory. To answer this question, one has to carefully treat the factors $\eta_{ \pm}$(7.13) in the integration over the internal times of the Cooperon. As shown in Appendix E the classical Cooperon trajectory is characterized by a single velocity and this is $u$. Having established the fact that there is the unique velocity on the whole classical trajectory of the Cooperon, we can, in the leading approximation, ignore below the difference between $u$ and $v_{F}$ in the functions $M\left(N, N^{\prime}\right)$.

Neglecting first the disorder-induced corrections $b(x, \tau)$ in Eq. (7.32) and summing up according to Eqs. (7.37),(7.38) all 12 terms each of which is given by Eq. (7.39), we observe that the difference $M_{f f}-M_{f b}$ in this approximation "miraculously" cancels out to zero on the classical trajectory. This is in agreement with Eq. (6.14) saying that the dephasing rate for the Cooperon vanishes in the limit $\gamma \rightarrow 0$.

Combining Eqs. (7.32) and (7.39), the disorderrenormalized correlators $M$ are represented as

$$
M=\left.M\right|_{\gamma=0}+4 b,
$$

where $b=b_{1}+b_{2}$ is given by Eqs. (7.30), (7.30). In order to evaluate the dephasing action on the classical trajectory, we analytically continue $b_{1,2}(x, \tau)$ in the complex plane of $\tau$. The analytical continuation of $b_{2}(x, \tau)$ is effected via Eq. (7.28). On the imaginary axis of $\tau$, the functions $b_{1,2}$ at order $\mathcal{O}\left(\gamma \alpha^{2}\right)$ read

$$
\begin{aligned}
b_{1}(x, i t) & \simeq \frac{\pi}{4} \frac{\alpha^{2} T}{v_{F}^{2}} \gamma|x|\left[\left(|x|-v_{F} t\right) \Theta\left(-|x|+v_{F} t\right)\right. \\
& \left.+\left(|x|+v_{F} t\right) \Theta\left(-|x|-v_{F} t\right)\right] \\
b_{2}(x, i t) & \simeq-\frac{\pi}{8} \frac{\alpha^{2} T}{v_{F}^{2}} \gamma\left[\left(|x|-v_{F} t\right)^{2} \Theta\left(-|x|+v_{F} t\right)\right. \\
& \left.+\left(|x|+v_{F} t\right)^{2} \Theta\left(-|x|-v_{F} t\right)\right]
\end{aligned}
$$

for both $|x| / v_{F}$ and $|t| \gg 1 / T$. Taking this limit is justified for the calculation of the dephasing rate since upon averaging over the positions of impurities in Eq. (6.4) the characteristic distance between impurities is $v_{F} \tau_{\phi}^{\mathrm{wl}}$ or larger [in fact it is spread over the range between $v_{F} \tau_{\phi}^{\mathrm{wl}}$ and $v_{F} \tau$ because of the logarithmic factor in Eq.(6.13)], which in turn is much larger than $v_{F} / T$ according to Eq. (6.14). The sum of Eqs. (7.52), (7.53) gives

$$
\begin{aligned}
& b(x, i t) \simeq \frac{\pi}{8} \frac{\alpha^{2} T}{v_{F}^{2}} \gamma\left(x^{2}-v_{F}^{2} t^{2}\right) \\
& \times\left[\Theta\left(-|x|+v_{F} t\right)+\Theta\left(-|x|-v_{F} t\right)\right] .
\end{aligned}
$$

Introducing a short-hand notation

$$
\beta\left(N, N^{\prime}\right)=\frac{8}{\pi \alpha^{2} T \gamma} b\left[x_{N}-x_{N^{\prime}}, i\left(t_{N}-t_{N^{\prime}}\right)\right],
$$

where $b$ is given by the asymptote (17.54), we see that $\beta\left(N, N^{\prime}\right)=0$ on the classical trajectory of the Cooperon for all $N, N^{\prime}$ except for

$$
\beta(1, \overline{2})=\beta(2, \overline{3})=-2 t_{a}\left(t_{c}-2 t_{a}\right) .
$$

Summing up the terms (7.56) with the signs prescribed by Eqs. (7.38), (7.51) we obtain the dephasing action:

$$
S_{C}^{\mathrm{deph}} \simeq 2 \pi \alpha^{2} T \gamma t_{a}\left(t_{c}-2 t_{a}\right) .
$$

This result coincides with Eq. (6.12) for the dephasing action derived via the path-integral calculation in Sec. VID, The integration along the cuts around the classical trajectory yields the renormalization of disorder on short spacial scales of order $v_{F} / T$ around impurities, similarly to the renormalization of the diffuson ladder in Eqs. (7.46), (7.49). The three-impurity Cooperon with both the renormalization of disorder and the dephasing included is thus given by

$$
c_{3} \simeq c_{3}^{(0)}(\Lambda / \pi T)^{6 \alpha} \exp \left[-2 \pi \alpha^{2} T \gamma t_{a}\left(t_{c}-2 t_{a}\right)\right],
$$

where

$$
\begin{aligned}
c_{3}^{(0)} & =\left(\frac{\gamma_{0}}{2 v_{F}}\right)^{3} \frac{\left(T / 2 v_{F}\right)^{2}}{\sinh ^{2}\left[\pi T\left(x_{\Sigma} / v_{F}+i \tau\right)\right]} \\
& \times\left[\Theta\left(x_{1}\right) \Theta\left(x_{1}-x_{2}\right) \Theta\left(x_{3}-x_{2}\right)\right. \\
& \times \Theta\left(x_{3}-x\right) \Theta\left(x_{3}\right) \Theta\left(x_{1}-x\right) \\
& +\Theta\left(-x_{1}\right) \Theta\left(x_{2}-x_{1}\right) \Theta\left(x_{2}-x_{3}\right) \\
& \left.\times \Theta\left(x-x_{3}\right) \Theta\left(-x_{3}\right) \Theta\left(x-x_{1}\right)\right]
\end{aligned}
$$


is the three-impurity Cooperon bubble without interaction,

$$
x_{\Sigma}=2\left(x_{1}+x_{3}-x_{2}\right)-x
$$

$\left|x_{\Sigma}\right|$ is the total length of the classical trajectory along which the density fluctuations propagate from point $x=0$ to point $x$. The length of the Cooperon loop $v_{F} t_{c}$ and the distance between two right-most impurities $v_{F} t_{a}$ are related to the positions of impurities by $v_{F} t_{c} / 2=\max \left\{x_{3}, x_{1}\right\}-x_{2}$ and $v_{F} t_{a}=\left|x_{3}-x_{1}\right|$. Equation (7.58) describes the Cooperon bubble with scalar vertices - the current vertices are straightforwardly incorporated below.

To calculate the conductivity, one has to add also selfenergy corrections to the Green's functions constituting the Cooperon bubble. In the presence of e-e interaction, this is done in essentially the same way as the renormalization of impurities in Sec. VIIE and results in the additional factor $\exp \left(-\gamma\left|x_{\Sigma}\right| / v_{F}\right)$, where $\gamma$ is the renormalized rate of backscattering, which the Cooperon acquires on the length $\left|x_{\Sigma}\right|$. The WL correction $\Delta \sigma_{\mathrm{wl}}$ is then expressed in terms of $c_{3}$ from Eq. (7.58) as

$$
\begin{aligned}
& \Delta \sigma_{\mathrm{wl}}=-2 \times\left(\frac{1}{2}\right)^{2} \times\left\{\frac{e^{2} v_{F}^{2}}{\Omega_{n}} \int_{0}^{1 / T} d \tau \int d x e^{i \Omega_{n} \tau}\right. \\
& \left.\times \int d x_{1} \int d x_{2} \int d x_{3} c_{3} e^{-\gamma\left|x_{\Sigma}\right| / v_{F}}\right\}_{\Omega_{n} \rightarrow+0},
\end{aligned}
$$

where the factor of 2 accounts for the contribution of the diagrams (b) and (c) in Fig. 5. which give exactly the same contribution as the Cooperon, and the factor of $(1 / 2)^{2}$ comes from the dressing of the current-vertices by the diffuson ladders, similarly to Eq. (B.1). The current vertices in Eq. (7.61) are simply given by $\pm v_{F}$, since we formulate our model in terms of a linear electron spectrum from the very beginning.112 Integrating (7.61) we reproduce Eq. (6.13) which was obtained in Sec. VID in two distinct steps by treating the renormalization of disorder and dephasing separately. Here, we have demonstrated how these two effects arise "side by side" from the technical point of view in the framework of the functional bosonization. Specifically, the analytical structure of the density-density correlator in the time domain is such that the dephasing comes from the classical trajectory, whereas the renormalization of impurities is associated with the integration along strong branch cuts around the classical trajectory.

\section{SUMMARY}

In this paper, we have studied the transport properties of interacting spinless electrons in a disordered quantum wire within the framework of the Luttinger-liquid model. Our main result is the weak-localization correction (6.13), governed by the dephasing rate (6.14).
We have developed two alternative approaches for a systematic analytic treatment of the problem. One is a two-step procedure which combines the bosonic-RG treatment of high-energy renormalization processes at the first step with the subsequent analysis of low-energy real processes in the fermionic language. The other approach is based on the method of "functional bosonization" which makes it possible to treat both types of effects simultaneously.

We have demonstrated that the notion of weak localization is applicable to the strongly correlated onedimensional electron system. This finding is of conceptual importance, showing that the famous non-Fermiliquid character of the Luttinger liquid does not prevent this system from exhibiting the features characteristic of conventional mesoscopic electron structures. Our approach thus provides a framework for systematically studying the mesoscopic phenomena in strongly correlated electron systems. Further directions of research include mesoscopic fluctuations and noise in a disordered Luttinger liquid, spin-related effects (in particular, the magnetoresistance), several-channel quantum wires, transport in a disordered system in the limit of strong interaction, transport in a "granular Luttinger liquid" (strong impurities).

\section{ACKNOWLEDGMENTS}

We thank A. Altland, D. Aristov, T. Giamarchi, L. Glazman, M. Grayson, K. Le Hur, D. Maslov, A. Nersesyan, A. Tsvelik, A. Yashenkin, O. Yevtushenko, and I. Yurkevich for interesting discussions. The work was supported by the Schwerpunktprogramm "Quanten-HallSysteme" and the Center for Functional Nanostructures of the Deutsche Forschungsgemeinschaft, by RFBR, by the Program "Leading Russian Scientific Schools" (Grant No. 2192.2003.2), and by the Program of RAS "Condensed Matter Physics". The authors acknowledge the hospitality of the Condensed Matter and Statistical Physics Section of the Abdus Salam ICTP (Trieste), where part of this work was performed. The work of IVG, conducted as part of the project "Quantum Transport in Nanostructures" made under the EUROHORCS/ESF EURYI Awards scheme, was supported by funds from the Participating Organisations of EURYI and the EC Sixth Framework Programme. ADM acknowledges the hospitality of the Kavli Institute for Theoretical Physics at Santa Barbara during the completion of the manuscript and partial support by NSF under Grant No. PHY9907949 .

\section{APPENDIX A: POLARIZATION OPERATOR AND SCREENING IN DISORDERED WIRES}

In this Appendix, we derive the polarization operator and the dynamically screened RPA-interaction for a 
disordered $1 \mathrm{~d}$ electron system. As everywhere in the paper, we consider spinless electrons. The Luttinger-liquid power-law singularities only show up in the renormalized strength of disorder $\gamma$ and do not make any other difference in the derivation below.

We begin by considering the clean case. According to Dzyaloshinskii-Larkin theorem, $\stackrel{111}{=}$ the RPA equations for screened interaction are then exact. The propagators of interaction between right-movers $\left(V_{++}\right)$and between right- and left-movers $\left(V_{+-}\right)$obey

$$
\begin{aligned}
& V_{++}=g_{4}-g_{4} \Pi_{+} V_{++}-g_{2} \Pi_{-} V_{+-}, \\
& V_{+-}=g_{2}-g_{2} \Pi_{+} V_{++}-g_{4} \Pi_{-} V_{+-},
\end{aligned}
$$

where

$$
\Pi_{ \pm}\left(i \Omega_{n}, q\right)=\frac{1}{2 \pi} \frac{q}{q v_{F} \mp i \Omega_{n}}
$$

are the chiral polarization operators for right(+)/left(-) movers, $\Omega_{n}=2 \pi n T$ is the bosonic Matsubara frequency. The total polarization operator is a sum of the chiral terms:

$$
\Pi\left(i \Omega_{n}, q\right)=\rho \frac{q^{2} v_{F}^{2}}{q^{2} v_{F}^{2}+\Omega_{n}^{2}},
$$

where $\rho=1 / \pi v_{F}$ is the thermodynamic density of states, so that $\Pi(0, q)=\rho$. For later use we also introduce the two-particle propagator

$$
D_{ \pm}\left(i \Omega_{n}, q\right)=\frac{i \Omega_{n}}{\left|\Omega_{n}\right|} \frac{\pi \rho}{i \Omega_{n} \mp q v_{F}},
$$

which satisfies

$$
\begin{aligned}
& \int \frac{d p}{2 \pi} G_{ \pm}^{(0)}\left(i \epsilon_{m}+i \Omega_{n}, p+q\right) G_{ \pm}^{(0)}\left(i \epsilon_{m}, p\right)=D_{ \pm}\left(i \Omega_{n}, q\right) \\
& \times\left[\Theta\left(-\epsilon_{m}\right) \Theta\left(\epsilon_{m}+\Omega_{n}\right)+\Theta\left(\epsilon_{m}\right) \Theta\left(-\epsilon_{m}-\Omega_{n}\right)\right],
\end{aligned}
$$

where $G_{ \pm}^{(0)}\left(i \epsilon_{m}, p\right)=\left(i \epsilon_{m} \mp v_{F} p\right)^{-1}$ are the free Green's functions for a given chirality and the ultraviolet momentum cutoff is sent to infinity. The polarization operator and the two-particle propagator are related to each other via

$$
\Pi_{ \pm}\left(i \Omega_{n}, q\right)=\frac{\rho}{2}-\frac{\left|\Omega_{n}\right|}{2 \pi} D_{ \pm}\left(i \Omega_{n}, q\right) .
$$

The solution to Eqs. (A.1) and (A.2) for arbitrary $g_{2}$ and $g_{4} \operatorname{reads}^{27}$

$$
\begin{aligned}
V_{++} & =\left[g_{4}+\left(g_{4}^{2}-g_{2}^{2}\right) \frac{1}{2 \pi v_{F}} \frac{q v_{F}}{i \Omega_{n}+q v_{F}}\right] \\
& \times\left(\Omega_{n}^{2}+q^{2} v_{F}^{2}\right) /\left(\Omega_{n}^{2}+q^{2} u^{2}\right), \\
V_{+-} & =g_{2}\left(\Omega_{n}^{2}+q^{2} v_{F}^{2}\right) /\left(\Omega_{n}^{2}+q^{2} u^{2}\right),
\end{aligned}
$$

where

$$
u=v_{F}\left[\left(1+g_{4} / 2 \pi v_{F}\right)^{2}-\left(g_{2} / 2 \pi v_{F}\right)^{2}\right]^{1 / 2} .
$$

For $g_{4}=g_{2}$ we have

$$
\begin{aligned}
V_{++} & =V_{+-}=g_{2} \frac{\Omega_{n}^{2}+q^{2} v_{F}^{2}}{\Omega_{n}^{2}+u^{2} q^{2}}, \\
u & =v_{F} \sqrt{1+\frac{g_{2}}{\pi v_{F}}},
\end{aligned}
$$

while for $g_{4}=0$ the propagators $V_{++}$and $V_{+-}$are different from each other:

$$
\begin{aligned}
V_{++} & =\frac{g_{2}^{2}}{2 \pi v_{F}} \frac{q v_{F}\left(i \Omega_{n}-q v_{F}\right)}{\Omega_{n}^{2}+u^{2} q^{2}}, \\
V_{+-} & =g_{2} \frac{\Omega_{n}^{2}+q^{2} v_{F}^{2}}{\Omega_{n}^{2}+u^{2} q^{2}}, \\
u & =v_{F}\left[1-\left(g_{2} / 2 \pi v_{F}\right)^{2}\right]^{1 / 2} .
\end{aligned}
$$

The interaction between left-movers is given by $V_{++}$with $q \rightarrow-q$.

Let us now turn to the disordered case. We only take into account the backward scattering induced by disorder, since the forward scattering can be completely gauged out in the calculation of the conductivity. We average the RPA bubbles over disorder separately by using the ladder approximation, see Sec. VID The backscattering off disorder makes it necessary to introduce two indices $\mu, \nu= \pm$ in the polarization bubble $\Pi_{\mu \nu}$, corresponding to the chirality of the vertices of the bubble. The RPA equations for the interaction propagators in the presence of disorder read

$$
\begin{aligned}
V_{++} & =g_{4}-\left(g_{4} \Pi_{++}+g_{2} \Pi_{-+}\right) V_{++} \\
& -\left(g_{2} \Pi_{--}+g_{4} \Pi_{+-}\right) V_{+-}, \\
V_{+-} & =g_{2}-\left(g_{2} \Pi_{++}+g_{4} \Pi_{-+}\right) V_{+-} \\
& -\left(g_{4} \Pi_{--}+g_{2} \Pi_{+-}\right) V_{++},
\end{aligned}
$$

where

$$
\Pi_{\mu \nu}\left(i \Omega_{n}, q\right)=\frac{\rho}{2} \delta_{\mu \nu}-\frac{\left|\Omega_{n}\right|}{2 \pi} D_{\mu \nu}\left(i \Omega_{n}, q\right) .
$$

We now calculate the two-particle propagators $D_{\mu \nu}$ in the RPA. Performing the analytical continuation to real frequencies $i \Omega_{n} \rightarrow \omega+i 0$, we introduce the retarded twoparticle propagator $\left[D_{\mu \nu}^{R}(\omega, q)\right]^{(0)}$ with only self-energy impurity lines included,

$$
\left[D_{++}^{R}(\omega, q)\right]^{(0)}=\frac{i \pi \rho}{\omega-q v_{F}+i \gamma / 2},
$$

where

$$
\gamma=1 / \tau
$$

and $\tau$ is the transport scattering time. Note the factor of $1 / 2$ in front of $\gamma$ in Eq. (A.12), which reflects the fact that only backscattering off impurities is considered, i.e., $\tau_{q}=$ $2 \tau$, where $\tau_{q}$ is the quantum scattering time, in contrast to the case of isotropic scattering, where $\tau_{q}=\tau$. The 
propagator $\left[D_{--}^{R}(\omega, q)\right]^{(0)}$ is given by Eq. A.12 with the change $q \rightarrow-q$, whereas the nondiagonal propagators $\left[D_{\mu,-\mu}^{R}(\omega, q)\right]^{(0)}=0$.

The equations for dressed two-particle retarded propagators read

$$
\begin{aligned}
& D_{--}^{R}(\omega, q)=\left[D_{--}^{R}(\omega, q)\right]^{(0)}\left[1+\frac{\gamma}{2 \pi \rho} D_{+-}^{R}(\omega, q)\right], \\
& D_{+-}^{R}(\omega, q)=\left[D_{++}^{R}(\omega, q)\right]^{(0)} \frac{\gamma}{2 \pi \rho} D_{--}^{R}(\omega, q),
\end{aligned}
$$

and

$$
\begin{aligned}
& D_{++}^{R}(\omega, q)=D_{--}^{R}(\omega,-q), \\
& D_{+-}^{R}(\omega, q)=D_{-+}^{R}(\omega, q) .
\end{aligned}
$$

Solving Eqs. (A.14 - A.17), we get

$$
\begin{aligned}
& D_{--}^{R}(\omega, q)=i \pi \rho \frac{\omega-q+i \gamma / 2}{\omega^{2}-q^{2} v_{F}^{2}+i \omega \gamma}, \\
& D_{+-}^{R}(\omega, q)=-\pi \rho \frac{\gamma}{2} \frac{1}{\omega^{2}-q^{2} v_{F}^{2}+i \omega \gamma},
\end{aligned}
$$

and

$$
\begin{aligned}
\Pi_{--}(\omega, q) & =\Pi_{++}(\omega,-q) \\
& =\frac{\rho}{2} \frac{q^{2} v_{F}^{2}-q v_{F} \omega-i \omega \gamma / 2}{q^{2} v_{F}^{2}-\omega^{2}-i \omega \gamma}, \\
\Pi_{+-}(\omega, q) & =\Pi_{-+}(\omega, q) \\
& =\frac{\rho}{2} \frac{i \omega \gamma / 2}{q^{2} v_{F}^{2}-\omega^{2}-i \omega \gamma}, \\
\Pi(\omega, q) & =\sum_{\mu \nu} \Pi_{\mu \nu}=\rho \frac{q^{2} v_{F}^{2}}{q^{2} v_{F}^{2}-\omega^{2}-i \omega \gamma} .
\end{aligned}
$$

Equation A.22 reduces to Eq. A.3 in the clean limit $\gamma \rightarrow 0$ and acquires the conventional diffusive form

$$
\Pi^{\text {diff }}(\omega, q)=\rho D q^{2} /\left(D q^{2}-i \omega\right)
$$

in the limit $\omega \ll \gamma, q v_{F}$, where the $1 \mathrm{~d}$ diffusion constant $D=v_{F}^{2} / \gamma$.

From Eqs. (A.10), A.11, A.20, and A.21, we obtain the retarded interaction propagators for $g_{2}=g_{4}$ :

$$
\begin{aligned}
V_{++}^{R} & =V_{+-}^{R}=\frac{g_{2}}{1+g_{2} \Pi} \\
& =g_{2} \frac{q^{2} v_{F}^{2}-\omega^{2}-i \omega \gamma}{q^{2} u^{2}-\omega^{2}-i \omega \gamma}
\end{aligned}
$$

with $u$ given by Eq. (A.6). For $g_{4}=0$ we have

$$
\begin{aligned}
V_{++}^{R} & =-g_{2}^{2} \frac{\Pi_{--}}{\left(1+g_{2} \Pi_{+-}\right)^{2}-g_{2}^{2} \Pi_{--} \Pi_{++}} \\
& =-\frac{g_{2}^{2}}{2 \pi v_{F}} \frac{q^{2} v_{F}^{2}-q v_{F} \omega-i \omega \gamma / 2}{q^{2} u^{2}-\omega^{2}-i \omega(\gamma+\delta \gamma)},
\end{aligned}
$$

$$
\begin{aligned}
V_{+-}^{R} & =g_{2} \frac{1+g_{2} \Pi_{+-}}{\left(1+g_{2} \Pi_{+-}\right)^{2}-g_{2}^{2} \Pi_{--} \Pi_{++}} \\
& =g_{2} \frac{q^{2} v_{F}^{2}-\omega^{2}-i \omega(\gamma+\delta \gamma / 2)}{q^{2} u^{2}-\omega^{2}-i \omega(\gamma+\delta \gamma)},
\end{aligned}
$$

where $u$ is given by Eq. (A.9) and

$$
\delta \gamma=-\gamma \frac{g_{2}}{2 \pi v_{F}} .
$$

\section{APPENDIX B: WEAK-LOCALIZATION CORRECTION TO THE CONDUCTIVITY IN 1D}

In this Appendix, we calculate the WL correction to the conductivity in a noninteracting $1 \mathrm{~d}$ system, assuming that the phase coherence is broken by an external source of dephasing and the dephasing time is small; specifically, $\tau_{\phi} \ll \tau$. Under this condition, the conductivity can be expanded in powers of $\tau_{\phi} / \tau$. The lowest-order diagrams are depicted in Fig. 5. The sum of the diagrams with two impurity lines, shown in Fig. 7, yields a zero contribution to order $\left(\epsilon_{F} \tau\right)^{-1}$.

We formally consider only backward scattering, i.e., the impurity line corresponds to the correlation function of the backscattering random potential

$$
L^{-1}\left\langle\left|\mathcal{U}_{b}(q)\right|^{2}\right\rangle=1 / 2 \pi \rho \tau
$$

( $L$ is the system size) and changes chirality at every impurity vertices. For the anisotropic scattering, we have $\tau=\tau_{q} / 2$, where $\tau$ is the transport time and $\tau_{q}$ is the quantum lifetime. The retarded single-particle Green's function expressed in terms of $\tau$ reads

$$
G_{ \pm}^{R}(\epsilon, p)=\left(\epsilon \mp v_{F} p+i / 2 \tau_{q}\right)^{-1}=\left(\epsilon \mp v_{F} p+i / 4 \tau\right)^{-1} .
$$

The anisotropic scattering also introduces the current vertex renormalization: each current vertex is multiplied by a factor $\tau / \tau_{q}=1 / 2$.

The sum of the diagrams (b) and (c) in Fig. 5 with an impurity line covering the two-impurity Cooperon (two crossed impurity lines) is equal to the contribution $\sigma_{C 3}$ of the diagram with the three-impurity Cooperon. The total three-impurity WL correction is thus given by

$$
\Delta \sigma_{\mathrm{wl}}=2 \sigma_{C 3} .
$$

The expression for $\sigma_{C 3}$ reads

$$
\sigma_{C 3}=-2\left(\frac{\tau}{\tau_{q}}\right)^{2} e^{2} v_{F}^{2} \int \frac{d \epsilon}{2 \pi}\left(-\frac{\partial f_{\epsilon}}{\partial \epsilon}\right) \int \frac{d Q}{2 \pi} J(Q),
$$

where $f_{\epsilon}$ is the Fermi distribution function, the overall minus sign reflects the fact that the product of the current vertices is negative in the Cooperon with three backscattering lines, and the factor of 2 is related to two possibilities of setting chiralities at the vertices: $(+-)$ 
and $(-+)$. The function $J(Q)$ describes the fermionic loop:

$$
\begin{aligned}
& J(Q)=\frac{1}{(2 \pi \rho \tau)^{3}} \int \frac{d p}{2 \pi} \int \frac{d p_{1}}{2 \pi} \int \frac{d p_{2}}{2 \pi} \\
& \times G_{+}^{R}(p) G_{-}^{R}\left(p_{1}\right) G_{+}^{R}\left(p_{2}\right) G_{-}^{R}(-p+Q) \\
& \times G_{+}^{A}(p) G_{-}^{A}\left(-p_{2}+Q\right) G_{+}^{A}\left(-p_{1}+Q\right) G_{-}^{A}(-p+Q) .
\end{aligned}
$$

Using the equality

$$
G_{ \pm}^{R}(p) G_{ \pm}^{A}(p)=2 i \tau\left[G_{ \pm}^{R}(p)-G_{ \pm}^{A}(p)\right]
$$

we simplify Eq. (B.2):

$$
J(Q)=4 \tau^{2}|P(Q)|^{2}\left[P(Q)+P^{*}(Q)\right],
$$

where

$$
P(Q)=\frac{1}{2 \pi \rho \tau} \int \frac{d p}{2 \pi} G_{+}^{R}(p) G_{-}^{A}(-p+Q) .
$$

Now we introduce a phenomenological dephasing time $\tau_{\phi}$ through an additional decay of the Green's function:

$$
G_{ \pm}^{R}(\epsilon, p) \rightarrow\left(\epsilon \mp v_{F} q+i / 4 \tau+i / 2 \tau_{\phi}\right)^{-1}
$$

which yields

$$
\begin{aligned}
P(Q) & =\frac{1}{2 \tau} \frac{1}{i v_{F} Q+1 / 2 \tau+1 / \tau_{\phi}}, \\
J(Q) & =\frac{1}{\tau}\left(\frac{1}{2 \tau}+\frac{1}{\tau_{\phi}}\right) \frac{1}{\left[\left(v_{F} Q\right)^{2}+\left(1 / 2 \tau+1 / \tau_{\phi}\right)^{2}\right]^{2}}
\end{aligned}
$$

Substituting Eq. (B.4) in Eq. (B.1), we find for $\tau_{\phi} \ll \tau$ :

$$
\Delta \sigma_{\mathrm{wl}}=-\frac{1}{8} \sigma_{\mathrm{D}}\left(\frac{\tau_{\phi}}{\tau}\right)^{2}
$$

where $\sigma_{\mathrm{D}}=e^{2} v_{F} \tau / \pi$ is the Drude conductivity.

Equation (B.5) for the weak-localization correction can be cast in the form of an integral over the time needed for a particle to return to the starting point after two backscatterings:

$$
\Delta \sigma_{\mathrm{wl}}=-2 \sigma_{\mathrm{D}} \int_{0}^{\infty} d t_{c} P_{2}\left(t_{c}\right) \exp \left(-t_{c} / \tau_{\phi}\right)
$$

Here

$$
P_{2}(t) \simeq t / 16 \tau^{2}, \quad t \ll \tau
$$

is the return probability after two reflections (hence the subscript 2) for a particle which starts moving either to the right or to the left and the overall factor of 2 in Eq. (B.6) comes from the summation over the sign of the velocity at the starting point.

Let us now turn from the phenomenological factor $\exp \left(-t_{c} / \tau_{\phi}\right)$ to the actual dephasing factor. The latter depends not only on the total time $t_{c}$ but also on the geometry of the Cooperon path. We thus have to perform averaging over the geometry of the path at given $t_{c}$. For this purpose we need the probability density of return $P_{2}\left(t_{c}, t_{a}\right)$ for a particle which starts moving to the right at point $x=0$ under the condition that the total length is $v_{F} t_{c}$ and the first reflection occurs at point $x=v_{F} t_{a}$. This function is written as

$$
\begin{aligned}
& P_{2}\left(t_{c}, t_{a}\right)=v_{F} \int_{0}^{\infty} d t_{b} P_{1}\left(t_{a}\right) P_{1}\left(t_{b}-t_{a}\right) \Theta\left(t_{b}-t_{a}\right) \\
& \times \exp \left[-\left(t_{c}-t_{b}\right) / 2 \tau\right] \Theta\left(t_{c}-t_{b}\right) \delta\left[x\left(t_{c}\right)\right] .
\end{aligned}
$$

Here

$$
P_{1}(t)=\frac{d}{d t}\left(1-e^{-t / 2 \tau}\right)
$$

is the probability density of being reflected at point $v_{F} t$ (again, note that $\tau$ is the transport scattering time and only the backscattering is taken into account). The factor $\exp \left[-\left(t_{c}-t_{b}\right) / 2 \tau\right]$ in Eq. (B.7) describes the probability of avoiding the backscattering on the last segment of the path and

$$
x\left(t_{c}\right)=2 v_{F}\left(t_{a}-t_{b}\right)+v_{F} t_{c}
$$

for $t_{c}>t_{b}$. We obtain

$$
\begin{aligned}
& P_{2}\left(t_{c}, t_{a}\right)=\frac{1}{4 \tau^{2}} e^{-t_{c} / 2 \tau} \\
& \times \int_{0}^{\infty} d t_{b} \Theta\left(t_{b}-t_{a}\right) \Theta\left(t_{c}-t_{b}\right) \delta\left[2\left(t_{a}-t_{b}\right)+t_{c}\right] \\
& =\frac{1}{8 \tau^{2}} e^{-t_{c} / 2 \tau} \Theta\left(t_{c}-2 t_{a}\right) .
\end{aligned}
$$

For the dephasing action which depends on $t$ and $t_{a}$ we have

$$
\Delta \sigma_{\mathrm{wl}}=-2 \sigma_{\mathrm{D}} \int_{0}^{\infty} d t_{c} \int_{0}^{\infty} d t_{a} P_{2}\left(t_{c}, t_{a}\right) \exp \left[-S\left(t_{c}, t_{a}\right)\right],
$$

which reduces to Eq. (B.6) for the phenomenological action $S\left(t_{c}, t_{a}\right)=t_{c} / \tau_{\phi}$.

\section{APPENDIX C: DEPHASING ACTION IN THE PATH-INTEGRAL APPROACH}

In this Appendix, we present details of the calculation of the dephasing action defined by Eq. (6.6) and given in the final form by Eq. (6.12). For the imaginary parts of the retarded interaction propagators (A.24), A.25) which enter Eq. (6.6), we have, retaining only the terms of order $\mathcal{O}\left(g_{2}^{2}\right)$ :

$$
\operatorname{Im} V_{+ \pm}^{R}(\omega, q) \simeq-\frac{g_{2}^{2}}{4 \pi v_{F}} \omega \gamma \frac{\left(q v_{F}-\omega\right)\left(q v_{F} \mp \omega\right)}{\left(q^{2} v_{F}^{2}-\omega^{2}\right)^{2}+\omega^{2} \gamma^{2}} .
$$

Next, we introduce functions $\mathcal{F}_{+ \pm}(\omega, q)$ which obey

$$
\operatorname{Im} V_{+ \pm}^{R}(\omega, q)=-\frac{g_{2}^{2}}{4 \pi v_{F}} \omega \mathcal{F}_{+ \pm}(\omega, q) .
$$


Putting $g_{2}=0$ in $\mathcal{F}_{+ \pm}$and doing the Fourier transform to $(x, t)$ space, we get

$$
\begin{aligned}
\mathcal{F}_{++}(x, t) & =e^{-\gamma|t| / 2}\left[\delta\left(x_{+}\right)\right. \\
& \left.+\frac{\gamma^{2}\left|x_{-}\right|}{16 v_{F}^{2}} f_{++}\left(-\frac{\gamma^{2}}{16 v_{F}^{2}} x_{+} x_{-}\right)\right], \\
\mathcal{F}_{+-}(x, t) & =\frac{\gamma}{4 v_{F}} e^{-\gamma|t| / 2} f_{+-}\left(-\frac{\gamma^{2}}{16 v_{F}^{2}} x_{+} x_{-}\right) .
\end{aligned}
$$

Here $x_{ \pm}=x \pm v_{F} t$

$$
\begin{aligned}
f_{++}(z) & =\frac{1}{\sqrt{z}} I_{1}(2 \sqrt{z}) \Theta(z) \\
f_{+-}(z) & =I_{0}(2 z) \Theta(z)
\end{aligned}
$$

$I_{0}(z)$ and $I_{1}(z)$ are the modified Bessel functions. Since the dephasing action becomes of order unity at times $t \sim \tau_{\phi}^{\mathrm{wl}} \ll \tau$, we expand $\mathcal{F}_{++}(x, t)$ and $\mathcal{F}_{+-}(x, t)$ to the first order in the disorder strength $\gamma$, which yields Eqs. (6.9), (6.10).

Let us now turn to the calculation of the dephasing action $S\left(t, t_{a}\right)$ which can be represented as

$$
S\left(t, t_{a}\right)=S_{\mathrm{ff}}+S_{\mathrm{bb}}-S_{\mathrm{fb}}+S_{\mathrm{bf}}=2\left(S_{\mathrm{ff}}-S_{\mathrm{fb}}\right),
$$

where $S_{i j}$ are given by Eq. (6.6). We split the forward path into three segments corresponding to the right- $(\mathrm{R})$ and left- $(\mathrm{L})$ moving parts, where the coordinate $x_{f}(t)$ behaves as

$$
\begin{aligned}
& \mathrm{I}_{\mathrm{f}}, \quad R: \quad 0<t<t_{a} ; \quad x_{f}(t)=v_{F} t \\
& \mathrm{II}_{\mathrm{f}}, \quad L: \quad t_{a}<t<t_{a}+\frac{t_{c}}{2} ; \quad x_{f}(t)=v_{F}\left(2 t_{a}-t\right) \\
& \mathrm{III}_{\mathrm{f}}, \quad R: \quad t_{a}+\frac{t}{2}<t<t_{c} ; \quad x_{f}(t)=v_{F}\left(t-t_{c}\right) .
\end{aligned}
$$

Similarly, for the backward (time-reversed) path we have $x_{b}(t)=x_{f}\left(t_{c}-t\right)$ :

$$
\begin{aligned}
& \mathrm{I}_{\mathrm{b}}, \quad L: \quad 0<t<\frac{t_{c}}{2}-t_{a} ; \quad x_{b}(t)=-v_{F} t \\
& \mathrm{II}_{\mathrm{b}}, \quad R: \quad \frac{t_{c}}{2}-t_{a}<t<t_{c}-t_{a} ; \\
& \quad x_{b}(t)=v_{F}\left(2 t_{a}-t_{c}+t\right) \\
& \mathrm{III}_{\mathrm{b}}, \quad L: \quad t_{c}-t_{a}<t<t_{c} ; \quad x_{b}(t)=v_{F}\left(t_{c}-t\right) .
\end{aligned}
$$

The "self-energy" part $S_{\mathrm{ff}}$ of the action is written as

$$
S_{\mathrm{ff}}=\frac{g_{2}^{2}}{4 \pi v_{F}^{2}} T J_{\mathrm{ff}}, \quad J_{\mathrm{ff}}=\sum_{n=1}^{3} J_{n}+2 \sum_{n=4}^{6} J_{n},
$$

where each of the terms $J_{n}$ corresponds to the integration over a certain pair of the forward-path segments (for brevity, we set $v_{F}=1$ in the arguments of $\mathcal{F}_{\mu \nu}$ below):

$$
\mathrm{I}_{\mathrm{f}}-\mathrm{I}_{\mathrm{f}}: \quad J_{1}=v_{F} \int_{0}^{t_{a}} d t_{1} \int_{0}^{t_{a}} d t_{2}
$$

$$
\begin{aligned}
& \times \mathcal{F}_{++}\left(t_{1}-t_{2}, t_{1}-t_{2}\right), \\
& \mathrm{II}_{\mathrm{f}}-\mathrm{II}_{\mathrm{f}}: \quad J_{2}=v_{F} \int_{t_{a}}^{t_{a}+t_{c} / 2} d t_{1} \int_{t_{a}}^{t_{a}+t_{c} / 2} d t_{2} \\
& \times \mathcal{F}_{--}\left(-t_{1}+t_{2}, t_{1}-t_{2}\right), \\
& \mathrm{III}_{\mathrm{f}}-\mathrm{III}_{\mathrm{f}}: \quad J_{3}=v_{F} \int_{t_{a}+t_{c} / 2}^{t_{c}} d t_{1} \int_{t_{a}+t_{c} / 2}^{t_{c}} d t_{2} \\
& \times \mathcal{F}_{++}\left(t_{1}-t_{2}, t_{1}-t_{2}\right), \\
& \mathrm{I}_{\mathrm{f}}-\mathrm{II}_{\mathrm{f}}: \quad v_{F} \int_{0}^{t_{a}} d t_{1} \int_{t_{a}}^{t_{a}+t_{c} / 2} d t_{2} \\
& \times\left[\mathcal{F}_{+-}\left(t_{1}+t_{2}-2 t_{a}, t_{1}-t_{2}\right)\right. \\
&\left.+\mathcal{F}_{-+}\left(-t_{1}-t_{2}+2 t_{a}, t_{1}-t_{2}\right)\right], \\
& \mathrm{I}_{\mathrm{f}}-\mathrm{III}_{\mathrm{f}}: \quad v_{F} \int_{0}^{t_{a}} d t_{1} \int_{t_{a}+t_{c} / 2}^{t_{c}} d t_{2} \\
& 2 J_{5}=\left[\mathcal{F}_{++}\left(t_{1}-t_{2}+t_{c}, t_{1}-t_{2}\right)\right. \\
&\left.+\mathcal{F}_{++}\left(-t_{1}+t_{2}-t_{c}, t_{1}-t_{2}\right)\right], \\
& \mathrm{II}_{\mathrm{f}}-\mathrm{III}_{\mathrm{f}}: \quad v_{F} \int_{t_{a}}^{t_{a}+t_{c} / 2} d t_{1} \int_{t_{a}+t_{c} / 2}^{t_{c}} d t_{2} \\
& 2 J_{6}=\left[\mathcal{F}_{-+}\left(-t_{1}-t_{2}+2 t_{a}+t_{c}, t_{1}-t_{2}\right)\right. \\
&\left.+\mathcal{F}_{+-}\left(t_{1}+t_{2}-2 t_{a}-t_{c}, t_{1}-t_{2}\right)\right], \\
&
\end{aligned}
$$

The "vertex" part $S_{\mathrm{fb}}$ of the action is given by

$$
S_{\mathrm{fb}}=\frac{g_{2}^{2}}{4 \pi v_{F}^{2}} T J_{\mathrm{fb}}, \quad J_{\mathrm{fb}}=\sum_{n=7}^{15} J_{n},
$$

where

$$
\begin{aligned}
& \mathrm{I}_{\mathrm{f}}-\mathrm{I}_{\mathrm{b}}: \quad J_{7}=v_{F} \int_{0}^{t_{a}} d t_{1} \int_{0}^{t_{c} / 2-t_{a}} d t_{2} \\
& \times \mathcal{F}_{+-}\left(t_{1}+t_{2}, t_{1}-t_{2}\right), \\
& \mathrm{I}_{\mathrm{f}}-\mathrm{II}_{\mathrm{b}}: \quad J_{8}=v_{F} \int_{0}^{t_{a}} d t_{1} \int_{t_{c} / 2-t_{a}}^{t_{c}-t_{a}} d t_{2} \\
& \times \mathcal{F}_{++}\left(t_{1}-t_{2}-2 t_{a}+t_{c}, t_{1}-t_{2}\right) \text {, } \\
& \mathrm{I}_{\mathrm{f}}-\mathrm{III}_{\mathrm{b}}: \quad J_{9}=v_{F} \int_{0}^{t_{a}} d t_{1} \int_{t_{c}-t_{a}}^{t_{c}} d t_{2} \\
& \times \mathcal{F}_{+-}\left(t_{1}+t_{2}-t_{c}, t_{1}-t_{2}\right) \text {, } \\
& \mathrm{II}_{\mathrm{f}}-\mathrm{I}_{\mathrm{b}}: \quad J_{10}=v_{F} \int_{t_{a}}^{t_{a}+t_{c} / 2} d t_{1} \int_{0}^{t_{c} / 2-t_{a}} d t_{2} \\
& \times \mathcal{F}_{--}\left(-t_{1}+t_{2}+2 t_{a}, t_{1}-t_{2}\right) \text {, } \\
& \mathrm{II}_{\mathrm{f}}-\mathrm{II}_{\mathrm{b}}: \quad J_{11}=v_{F} \int_{t_{a}}^{t_{a}+t_{c} / 2} d t_{1} \int_{t_{c} / 2-t_{a}}^{t_{c}-t_{a}} d t_{2} \\
& \times \mathcal{F}_{-+}\left(-t_{1}-t_{2}+t_{c}, t_{1}-t_{2}\right) \text {, } \\
& \mathrm{II}_{\mathrm{f}}-\mathrm{III}_{\mathrm{b}}: \quad J_{12}=v_{F} \int_{t_{a}}^{t_{a}+t_{c} / 2} d t_{1} \int_{0}^{t_{c} / 2-t_{a}} d t_{2} \\
& \times \mathcal{F}_{--}\left(-t_{1}+t_{2}+2 t_{a}, t_{1}-t_{2}\right) \text {, } \\
& \mathrm{III}_{\mathrm{f}}-\mathrm{I}_{\mathrm{b}}: \quad J_{13}=v_{F} \int_{t_{c} / 2+t_{a}}^{t_{c}} d t_{1} \int_{0}^{t_{c} / 2-t_{a}} d t_{2} \\
& \times \mathcal{F}_{+-}\left(t_{1}+t_{2}-t_{c}, t_{1}-t_{2}\right) \text {, }
\end{aligned}
$$




$$
\begin{aligned}
\mathrm{III}_{\mathrm{f}}-\mathrm{II}_{\mathrm{b}}: \quad J_{14} & =v_{F} \int_{t_{c} / 2+t_{a}}^{t_{c}} d t_{1} \int_{t_{c} / 2-t_{a}}^{t_{c}-t_{a}} d t_{2} \\
& \times \mathcal{F}_{++}\left(t_{1}-t_{2}-2 t_{a}, t_{1}-t_{2}\right), \\
\mathrm{III}_{\mathrm{f}}-\mathrm{III}_{\mathrm{b}}: \quad J_{15} & =v_{F} \int_{t_{a}+t_{c} / 2}^{t_{c}} d t_{1} \int_{t_{c}-t_{a}}^{t_{c} / 2-t_{a}} d t_{2} \\
& \times \mathcal{F}_{+-}\left(t_{1}+t_{2}-2 t_{c}, t_{1}-t_{2}\right) .
\end{aligned}
$$

Carrying out the integration yields:

$$
\begin{aligned}
J_{1} & =\frac{t_{a}}{2} \\
J_{2} & =\frac{t_{c}}{4} \\
J_{3} & =\frac{1}{2}\left(\frac{t_{c}}{2}-t_{a}\right), \\
J_{4} & =\gamma \frac{t_{a} t_{c}}{8}, \\
J_{6} & =\gamma \frac{t_{c}}{8}\left(\frac{t_{c}}{2}-t_{a}\right), \\
J_{8} & =\frac{t_{a}}{2}-\gamma \frac{t_{a}}{4}\left(\frac{t_{c}}{2}-t_{a}\right), \\
J_{9} & =\gamma \frac{t_{a}^{2}}{4}, \\
J_{10} & =\frac{1}{2}\left(\frac{t_{c}}{2}-t_{a}\right)-\gamma \frac{t_{a}}{4}\left(\frac{t_{c}}{2}-t_{a}\right), \\
J_{14} & =\frac{1}{2}\left(\frac{t_{c}}{2}-t_{a}\right)-\gamma \frac{t_{a}}{4}\left(\frac{t_{c}}{2}-t_{a}\right), \\
J_{5} & =J_{7}=J_{15}=0 \\
J_{13} & =\frac{t_{c}}{2}\left(\frac{t_{c}}{2}-t_{a}\right)^{2}, \\
J_{12} & =\frac{t_{a}}{2}-\gamma \frac{t_{a}}{4}\left(\frac{t_{c}}{2}-t_{a}\right),
\end{aligned}
$$

and

$$
\begin{aligned}
J_{\mathrm{ff}} & =\frac{t_{c}}{2}+\gamma \frac{t_{c}^{2}}{8} \\
J_{\mathrm{fb}} & =\frac{t_{c}}{2}+\frac{\gamma}{4}\left[\frac{t_{c}^{2}}{2}-8 t_{a}\left(\frac{t_{c}}{2}-t_{a}\right)\right], \\
J_{\mathrm{ff}}-J_{\mathrm{fb}} & =2 \gamma t_{a}\left(\frac{t_{c}}{2}-t_{a}\right) .
\end{aligned}
$$

We see that the difference $J_{\mathrm{ff}}-J_{\mathrm{fb}}$ vanishes at $\gamma \rightarrow 0$, i.e., in the absence of disorder in the interaction propagators. Finally, we arrive at the expression for the total action

$$
\begin{aligned}
S\left(t, t_{a}\right) & =2 \times \frac{g_{2}^{2}}{4 \pi v_{F}^{2}} T\left(J_{\mathrm{ff}}-J_{\mathrm{fb}}\right) \\
& =\frac{g_{2}^{2}}{\pi v_{F}^{2}} T \gamma t_{a}\left(\frac{t_{c}}{2}-t_{a}\right) .
\end{aligned}
$$

Using $\alpha=g_{2} / 2 \pi v_{F}$, we obtain Eq. 6.12).

\section{APPENDIX D: FUNCTIONAL BOSONIZATION IN THE CLEAN CASE}

In this Appendix, we calculate the correlators $B_{\mu \nu}(x, \tau)$ in a clean Luttinger liquid for an arbitrary strength of interaction. Following the discussion in Sec. IIIA, we consider only the $g_{2}$ interaction, while the $g_{4}$ interaction is accounted for in the shift of the Fermi velocity, Eq. (3.12). Substituting the interaction propagator $V_{++}\left(i \Omega_{n}, q\right)$ given by Eq. A.7] [with $v_{F}$ understood here and below as the renormalized velocity (3.12)] in Eq. (7.8), we have

$$
\begin{aligned}
& B_{++}(x, \tau)=-T \sum_{n} \int \frac{d q}{2 \pi} \frac{g_{2}^{2}}{2 \pi v_{F}} \frac{q v_{F}}{\Omega_{n}^{2}+q^{2} u^{2}} \\
& \times \frac{e^{i q x-i \Omega_{n} \tau}-1}{-i \Omega_{n}+q v_{F}}=\mathcal{B}_{0}+\mathcal{B}_{>}+\mathcal{B}_{<},
\end{aligned}
$$

where $\mathcal{B}_{0}(x), \mathcal{B}_{>}(x, \tau)$, and $\mathcal{B}_{<}(x, \tau)$ are the contributions of terms with $\Omega_{n}=0, \Omega_{n}>0$, and $\Omega_{n}<0$ in the Matsubara sum over the bosonic frequency $\Omega_{n}=2 \pi n T$, respectively. The sums over $\Omega_{n}>0$ and $\Omega_{n}<0$ are related to each other as

$$
\mathcal{B}_{<}(x, \tau)=\mathcal{B}_{>}(-x,-\tau)
$$

To accurately treat the ultraviolet cutoff for an arbitrary ratio $u / v_{F}$, we introduce a finite range of interaction $d$, so that $g_{2}(q)$ depends on the transferred momentum $q$ and vanishes for $q d \gg 1$. The RPA approximation, leading to Eq. (D.1), is exact in the Luttinger-liquid model for arbitrary $g_{2}(q)$. In the integrand of Eq. (D.1), $g_{2}(q), v_{F}(q)$, and $u(q)$ are related to each other at given $q$ in precisely the same way, Eq. (4.2), as in the case of $g_{2}(q)=$ const. Everywhere below in this Appendix, except for Eq. (D.6), when writing $g_{2}, v_{F}$, or $u$, we understand them as taken at $q=0$.

The term in Eq. (D.1) with $\Omega_{n}=0$ at $|x| \gg d$ reads

$$
\mathcal{B}_{0}(x)=\frac{v_{F}^{2}-u^{2}}{2 u^{2}} \frac{2 \pi T}{v_{F}}|x| .
$$

In the integration over $q$ at $\Omega_{n}>0$, there are poles at $q=i \Omega_{n} / v_{F}$ and $q= \pm i \Omega_{n} / u$. The first only contributes to the integral if $\Omega_{n} \lesssim v_{F} / d$, whereas the latter-only if $\Omega_{n} \lesssim u / d$, which sets the limits of summation over $n$ :

$$
\begin{aligned}
& \mathcal{B}_{>}(x, \tau)=2 \pi T \Theta(x) \sum_{n=1}^{\infty} \frac{e^{-\Omega_{n} / \Lambda_{v}}\left[e^{-\Omega_{n}\left(x / v_{F}+i \tau\right)}-1\right]}{\Omega_{n}} \\
& -\pi T\left(\frac{v_{F}}{u}+\operatorname{sgn} x\right) \sum_{n=1}^{\infty} \frac{e^{-\Omega_{n} / \Lambda}\left[e^{-\Omega_{n}(|x| / u+i \tau)}-1\right]}{\Omega_{n}},
\end{aligned}
$$

where there appear two ultraviolet scales $\Lambda_{v}=v_{F} / d$ and $\Lambda=u / d$ and we approximate the cutoffs by the exponential factors $\exp \left(-\Omega_{n} / \Lambda_{v}\right)$ and $\exp \left(-\Omega_{n} / \Lambda\right)$ (the exact shape of the cutoffs plays no role in the infrared physics we deal with in this paper). 
Using for $N \gg 1$ and $\operatorname{Re} z \gg 1 / N$ the formula

$$
\sum_{n=1}^{N} \frac{e^{-n z}-1}{n} \simeq-\ln \left(1-e^{-z}\right)-\ln N
$$

we write $\mathcal{B}_{>}(x, \tau)$ for $|x| \gg d$ as

$$
\begin{aligned}
\mathcal{B}_{>}(x, \tau) & =\Theta(x) \ln \left\{\frac{\Lambda}{\Lambda_{v}} \frac{1-\exp [-2 \pi T(x / u+i \tau)]}{1-\exp \left[-2 \pi T\left(x / v_{F}+i \tau\right)\right]}\right\} \\
& -\frac{v_{F}-u}{2 u} \ln \frac{2 \pi T / \Lambda}{1-\exp [-2 \pi T(|x| / u+i \tau)]} .
\end{aligned}
$$

Combining Eqs. (D.3), (D.5), and (D.2), we obtain $B_{++}(x, \tau)$ given by Eq. (7.9). Note that the factor $v_{F} / u$ in $\eta_{+}(x, \tau)$ [Eq. [7.13)] comes from the ratio $\Lambda / \Lambda_{v}$ in Eq. (D.5). The remaining cutoff in $\mathcal{L}(x, \tau)$ is given by $\Lambda$.

The Green's function [Eq. (7.1)] of a right-mover then reads for $|x| \gg d$ :

$$
\begin{aligned}
& g_{+}(x, \tau)=g_{+}^{(0)}(x, \tau) \exp \left[-B_{++}(x, \tau)\right] \\
& =-\frac{i T}{2 u} \frac{1}{\sinh [\pi T(x / u+i \tau)]} \\
& \times \frac{(\pi T / \Lambda)^{\alpha_{b}}}{\{\sinh [\pi T(x / u+i \tau)] \sinh [\pi T(x / u-i \tau)]\}^{\alpha_{b} / 2}} .
\end{aligned}
$$

Note that the Fermi velocity $v_{F}$ drops out completely in the product $g_{+}^{(0)}(x, \tau) \eta_{+}(x, \tau)$. The Green's function in the real-time domain, Eq. (5.16), is obtained by means of the Wick rotation $\tau \rightarrow i t+\operatorname{sgn} t / \Lambda$.

The calculation of $B_{+-}(x, \tau)$ is performed in a similar way: substituting Eq. (A.8) in Eq. (7.8), we get

$$
B_{+-}(x, \tau)=-T \sum_{n} \int \frac{d q}{2 \pi} g_{2} \frac{e^{i q x-i \Omega_{n} \tau}-1}{q^{2} u^{2}+\Omega_{n}^{2}} .
$$

Note that the denominator $\Omega_{n}^{2}+q^{2} v_{F}^{2}$ in Eq. (7.8) is canceled by the numerator in Eq. (A.8), so that $v_{F}$ disappears from the problem right away and the integration over $q$ in Eq. (D.6) is determined at $\Omega_{n} \neq 0$ solely by the plasmon poles $q= \pm i \Omega_{n} / u$. For $|x| \gg d$, we arrive at Eq. (7.10).

In Sec. VII and in Appendix E, we do contour integrals in the complex plane of the Matsubara time $\tau$ and should be careful about the behavior of $B_{\mu \nu}(x, \tau)$ at $x \rightarrow 0$ for $\tau \rightarrow 0$. When integrating in the complex plane of $\tau$, it is convenient to have the ultraviolet cutoffs in $B_{\mu \nu}(x, \tau)$ that are independent of $\tau$. Our choice is to use Eq. (D.4), which yields a cutoff which depends on $x$ :

$$
x \rightarrow x+\frac{u}{\Lambda} \operatorname{sgn} x=x+d \operatorname{sgn} x,
$$

i.e., $|x| \rightarrow|x|+d$ in the propagators $B_{\mu \nu}(x, \tau)$ in Eqs. (7.9) (7.10), and similarly in the disorder-induced corrections to them in Eqs. (7.30), (7.30).

\section{APPENDIX E: "DIRTY RPA" IN THE FUNCTIONAL BOSONIZATION}

In this Appendix, we demonstrate that the correlators (7.7) in the presence of disorder are modified according to Eq. (7.8) with the effective RPA interaction $V_{\mu \nu}$ (7.20), (7.21) whose dynamical properties reflect the disorder-induced backscattering ("dirty RPA"). For this purpose, we directly calculate the disorder-induced correction to the averages of the type $\left\langle\exp \left\{i\left[\theta_{\mu}(x, \tau)-\theta_{\nu}(0,0)\right]\right\}\right\rangle$ in the framework of the diagrammatic technique formulated in Sec. VIIB In the expansion in powers of $\gamma$, the rules of the technique prescribe that impurity vertices are connected with each other via the correlators $\left.B_{\mu \nu}(x, \tau)\right|_{\gamma=0}$ in a spatially homogeneous system. To obey Wick's theorem, multiple fermionic loops can only be present in this technique if each of them contains backscattering vertices, since all fermionic loops without impurities have been accounted for by the "clean RPA" propagators. We show below that the result for $B_{\mu \nu}$ obtained this way coincides for $\alpha \ll 1$ with the result of the "dirty RPA", Eq. (7.32).

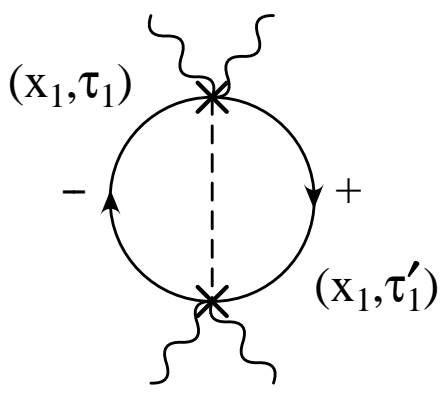

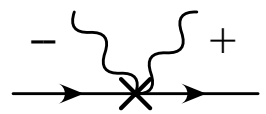

$(0,0)$

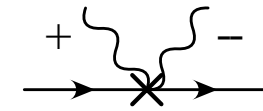

$(\mathrm{x}, \tau)$
FIG. 23: Diagram in the functional-bosonization technique, which describes the addition of disorder in the RPA interaction propagator. Two backscattering vertices $(0,0)$ and $(x, \tau)$ are connected to each other via the fermionic bubble with a single impurity at point $x_{1}$.

Let us consider the average of first order in $\gamma$,

$$
\begin{aligned}
& \left\langle e^{i\left[\theta_{+}(x, \tau)-\theta_{+}(0,0)\right]}\right\rangle-\left\langle e^{i\left[\theta_{+}(x, \tau)-\theta_{+}(0,0)\right]}\right\rangle_{\gamma=0} \\
& \simeq \int d x_{1} I\left(x, \tau, x_{1}\right),
\end{aligned}
$$

given by the diagram in Fig. 23 . We are interested in the pairings between the fields $\theta_{+}(0,0)$ and $\theta_{+}(x, \tau)$ that connect up the points $(0,0)$ and $(x, \tau)$ via the fermionic bubble that contains two backscatterings at point $x_{1}$ :

$$
I\left(x, \tau, x_{1}\right)=\frac{v_{F} \gamma_{0}}{2} e^{-B_{++}(x, \tau)} \int_{0}^{1 / T} d \tau_{1} \int_{0}^{1 / T} d \tau_{1}^{\prime}
$$




$$
\begin{aligned}
& \times g_{+}^{(0)}\left(0, \tau_{1}^{\prime}-\tau_{1}\right) g_{-}^{(0)}\left(0, \tau_{1}-\tau_{1}^{\prime}\right) \\
& \times e^{-B_{++}\left(0, \tau_{1}-\tau_{1}^{\prime}\right)-B_{--}\left(0, \tau_{1}-\tau_{1}^{\prime}\right)+2 B_{+-}\left(0, \tau_{1}-\tau_{1}^{\prime}\right)} \\
& \times\left[e^{-B_{++}\left(x_{1}, \tau_{1}^{\prime}\right)+B_{+-}\left(x_{1}, \tau_{1}^{\prime}\right)+B_{++}\left(x_{1}, \tau_{1}\right)-B_{+-}\left(x_{1}, \tau_{1}\right)}-1\right] \\
& \times\left[e^{B_{++}\left(x-x_{1}, \tau-\tau_{1}^{\prime}\right)-B_{+-}\left(x-x_{1}, \tau-\tau_{1}^{\prime}\right)}\right. \\
& \left.\times e^{-B_{++}\left(x-x_{1}, \tau-\tau_{1}\right)+B_{+-}\left(x-x_{1}, \tau-\tau_{1}\right)}-1\right] .
\end{aligned}
$$

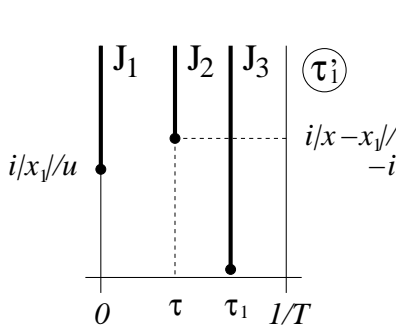

a)

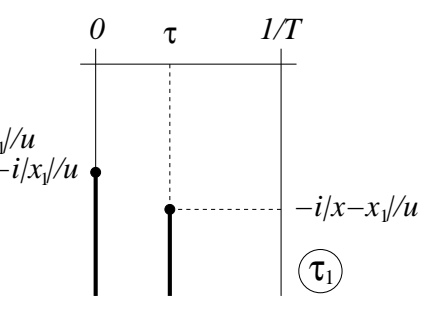

b)
FIG. 24: Analytical structure of the integrand in Eq. (E.2) in the complex plane of $\tau_{1}^{\prime}$ and $\tau_{1}$. (a) The contour of integration over $\tau_{1}^{\prime}$ is closed upwards and runs around three branch cuts $\left[J_{1,2,3}\right.$ are the corresponding terms in Eq. (E.4)]. (b) The contour of integration over $\tau_{1}$ for the first two terms in Eq. (E.4) is closed downwards and runs around two branch cuts.

All functions $B_{\mu \nu}$ in Eq. (E.2) are understood as taken at zero disorder. The analytical structure of the integrand in the complex plane of $\tau_{1}$ and $\tau_{1}^{\prime}$ is similar to that in the integrals analyzed in Secs. VIIEIVIIF, Specifically, in the plane of $\tau_{1}^{\prime}$ we have six vertical branch cuts starting at $\tau_{1}^{\prime}= \pm i\left|x_{1}\right| / u, \tau_{1}^{\prime}= \pm i\left|x-x_{1}\right| / u$, and $\tau_{1}^{\prime}=\tau_{1} \pm i / \Lambda$, and going to $\operatorname{Im} \tau_{1}^{\prime}= \pm i \infty$, Fig. 24a. On top of that, there are singularities associated with the functions $\eta_{\mu}$ (7.13). Note that the strong singularity (a double pole if $\alpha \rightarrow 0$ ) at $\tau_{1}^{\prime}=\tau_{1}$ (if $\Lambda$ is sent to infinity) that comes from the product of two free Green's functions $g_{ \pm}^{(0)}\left[0, \pm\left(\tau_{1}^{\prime}-\tau_{1}\right)\right]$ is largely canceled by the vanishing of the rest of the integrand at this same point. Evaluation of the integrals (E.2) at arbitrary $\alpha$ leads to rather cumbersome expressions which are not particularly interesting for our purposes. Let us extract the factor that renormalizes the impurity strength,

$$
\begin{aligned}
I\left(x, \tau, x_{1}\right)= & -\frac{v_{F} \gamma_{0}}{2 u^{2}}\left(\frac{\Lambda}{\pi T}\right)^{2 \alpha_{e}} e^{-\left.B_{++}(x, \tau)\right|_{\gamma=0}} \\
& \times J\left(x, \tau, x_{1}\right)
\end{aligned}
$$

and analyze the behavior of the factor $J\left(x, \tau, x_{1}\right)$ at small $\alpha$. By introducing a short-hand notation $s(z)=$ $\sinh (\pi T z), J\left(x, \tau, x_{1}\right)$ is written as

$$
\begin{aligned}
& J\left(x, \tau, x_{1}\right)=-\left(\frac{T}{2}\right)^{2} \int_{0}^{1 / T} d \tau_{1} \int_{0}^{1 / T} d \tau_{1}^{\prime} \\
& \times \frac{1}{s^{1-\alpha_{e}}\left[i\left(\tau_{1}^{\prime}-\tau_{1}\right)\right] s^{1-\alpha_{e}}\left[-i\left(\tau_{1}^{\prime}-\tau_{1}\right)\right]} \\
& \times\left\{\left[\frac{s\left(\left|x_{1}\right| / u+i \tau_{1}^{\prime}\right) s\left(\left|x_{1}\right| / u-i \tau_{1}^{\prime}\right)}{s\left(\left|x_{1}\right| / u+i \tau_{1}\right) s\left(\left|x_{1}\right| / u-i \tau_{1}\right)}\right]^{\alpha_{e} / 2} \chi_{1}-1\right\} \\
& \times\left\{\left[\frac{s\left(\left|x-x_{1}\right| / u+i \tau-i \tau_{1}\right)}{s\left(\left|x-x_{1}\right| / u+i \tau-i \tau_{1}^{\prime}\right)}\right]^{\alpha_{e} / 2}\right. \\
& \left.\times\left[\frac{s\left(\left|x-x_{1}\right| / u-i \tau+i \tau_{1}\right)}{s\left(\left|x-x_{1}\right| / u-i \tau+i \tau_{1}^{\prime}\right)}\right]^{\alpha_{e} / 2} \chi_{2}-1\right\},
\end{aligned}
$$

where

$$
\begin{aligned}
\chi_{1} & =\frac{s\left(x_{1} / v_{F}+i \tau_{1}^{\prime}\right) s\left(x_{1} / u+i \tau_{1}\right)}{s\left(x_{1} / u+i \tau_{1}^{\prime}\right) s\left(x_{1} / v_{F}+i \tau_{1}\right)}, \\
\chi_{2} & =\frac{s\left[\left(x-x_{1}\right) / v_{F}+i\left(\tau-\tau_{1}\right)\right]}{s\left(\left(x-x_{1}\right) / u+i\left(\tau-\tau_{1}\right)\right]} \\
& \times \frac{s\left[\left(x-x_{1}\right) / u+i\left(\tau-\tau_{1}^{\prime}\right)\right]}{s\left[\left(x-x_{1}\right) / v_{F}+i\left(\tau-\tau_{1}^{\prime}\right)\right]} .
\end{aligned}
$$

In Sec. VIIC, while calculating the disorder-induced correction to $B_{\mu \nu}$ to order $\mathcal{O}\left(\gamma \alpha^{2}\right)$, we neglected the difference between $v_{F}$ and $u$ in the interaction propagators. To compare with the result of Sec. VIIC we neglect the difference between the two velocities here as well; in particular, by putting $\chi_{1}=\chi_{2}=1$. We discuss the role of such factors in the calculation of the dephasing rate in Appendix $\mathrm{F}$

The integral over $\tau_{1}^{\prime}$ in Eq. (E.3) can be represented as a sum of three terms,

$$
J=-\frac{T}{2} \int_{0}^{1 / T} d \tau_{1}\left(J_{1}+J_{2}+J_{3}\right)
$$

each of which is the integral around a branch cut in the complex plane of $\tau_{1}^{\prime}$, Fig. 24k. Inspection of Eq. (E.3) shows that $J_{3}$, the term related to the branch cut starting at $\tau_{1}^{\prime}=\tau_{1}+i / \Lambda$, can be omitted for $\alpha_{e} \ll 1$, since $J_{3} \sim \mathcal{O}\left(\alpha_{e}^{3}\right)$, which gives a subleading contribution to $J$ [one power of $\alpha_{e}$ comes from the difference of the integrand on two sides of the cut and two more come from the expansion of the expressions in the curly brackets in Eq. (E.3) in $\tau_{1}^{\prime}-\tau_{1}$ ]. The integrals $J_{1,2}$ are written for $\alpha_{e} \ll 1$ as 


$$
\begin{aligned}
& J_{1} \simeq \alpha_{e} \frac{\pi T}{2} \int_{0}^{\infty} d t_{1}^{\prime} \frac{1}{s^{2\left(1-\alpha_{e}\right)}\left(t_{1}^{\prime}+\frac{\left|x_{1}\right|}{v_{F}}+i \tau_{1}\right)}\left[\frac{s\left(t_{1}^{\prime}\right) s\left(t_{1}^{\prime}+2 \frac{\left|x_{1}\right|}{v_{F}}\right)}{s\left(\frac{\left|x_{1}\right|}{v_{F}}+i \tau_{1}\right) s\left(\frac{\left|x_{1}\right|}{v_{F}}-i \tau_{1}\right)}\right]^{\alpha_{e} / 2} \\
& \times\left\{\left[\frac{s\left(\frac{\left|x-x_{1}\right|}{v_{F}}+i \tau-i \tau_{1}\right) s\left(\frac{\left|x-x_{1}\right|}{v_{F}}-i \tau+i \tau_{1}\right)}{s\left(t_{1}^{\prime}+\frac{\left|x-x_{1}\right|}{v_{F}}+\frac{\left|x_{1}\right|}{v_{F}}+i \tau\right) s\left(t_{1}^{\prime}-\frac{\left|x-x_{1}\right|}{v_{F}}+\frac{\left|x_{1}\right|}{v_{F}}+i \tau\right)}\right]^{\alpha_{e} / 2} e^{i \pi \alpha_{e} / 2}-1\right\}, \\
& \left.\left.J_{2} \simeq-\alpha_{e} \frac{\pi T}{2} \int_{0}^{\infty} d t_{1}^{\prime} \frac{s\left(\frac{\left|x-x_{1}\right|}{v_{F}}+i \tau-i \tau_{1}\right) s\left(\frac{\left|x-x_{1}\right|}{v_{F}}-i \tau+i \tau_{1}\right)}{s^{2\left(1-\alpha_{e}\right)}\left(t_{1}^{\prime}+\frac{\left|x-x_{1}\right|}{v_{F}}+i \tau_{1}-i \tau\right)}\right]\right]^{\alpha_{e} / 2} \\
& \times\left\{\left[\frac{s\left(t_{1}^{\prime}\right) s\left(t_{1}^{\prime}+2 \frac{\left|x-x_{1}\right|}{v_{F}}\right)}{s\left(\frac{\left|x_{1}\right|}{v_{F}}+i \tau_{1}\right) s\left(\frac{\left|x_{1}\right|}{v_{F}}-i \tau_{1}\right)}\right]^{\alpha_{e} / 2}\right),
\end{aligned}
$$

In turn, each of the integrals of $J_{1}$ and $J_{2}$ over $\tau_{1}$ can be split into a sum of two integrals along branch cuts in the complex plane of $\tau_{1}$ (Fig. 24b), so that in total we have $J \simeq\left(J_{1 a}+J_{1 b}\right)+\left(J_{2 a}+J_{2 b}\right)$, where for $\alpha_{e} \ll 1$ :

$$
\begin{aligned}
& J_{1 a} \simeq-\alpha_{e}^{2}\left(\frac{\pi T}{2}\right)^{2} \int_{0}^{\infty} d t_{1} \int_{0}^{\infty} d t_{1}^{\prime} \frac{1}{s^{2\left(1-\alpha_{e}\right)}\left(t_{1}+t_{1}^{\prime}+2 \frac{\left|x_{1}\right|}{v_{F}}\right)}\left[\frac{s\left(t_{1}^{\prime}\right) s\left(t_{1}^{\prime}+2 \frac{\left|x_{1}\right|}{v_{F}}\right)}{s\left(t_{1}\right) s\left(t_{1}+2 \frac{\left|x_{1}\right|}{v_{F}}\right)}\right]^{\alpha_{e} / 2} \\
& \times\left\{\left[\frac{s\left(t_{1}+\frac{\left|x-x_{1}\right|}{v_{F}}+\frac{\left|x_{1}\right|}{v_{F}}-i \tau\right) s\left(t_{1}-\frac{\left|x-x_{1}\right|}{v_{F}}+\frac{\left|x_{1}\right|}{v_{F}}-i \tau\right)}{s\left(t_{1}^{\prime}+\frac{\left|x-x_{1}\right|}{v_{F}}+\frac{\left|x_{1}\right|}{v_{F}}+i \tau\right) s\left(t_{1}^{\prime}-\frac{\left|x-x_{1}\right|}{v_{F}}+\frac{\left|x_{1}\right|}{v_{F}}+i \tau\right)}\right]^{\alpha_{e} / 2}-1\right\}, \\
& J_{1 b} \simeq \alpha_{e}^{2}\left(\frac{\pi T}{2}\right)^{2} \int_{0}^{\infty} d t_{1} \int_{0}^{\infty} d t_{1}^{\prime} \frac{1}{s^{2\left(1-\alpha_{e}\right)}\left(t_{1}+t_{1}^{\prime}+\frac{\left|x_{1}\right|}{v_{F}}+\frac{\left|x-x_{1}\right|}{v_{F}}+i \tau\right)}\left[\frac{s\left(t_{1}^{\prime}\right)}{s\left(t_{1}+\frac{\left|x_{1}\right|}{v_{F}}+\frac{\left|x-x_{1}\right|}{v_{F}}+i \tau\right)}\right]^{\alpha_{e} / 2} \\
& \times\left[\frac{s\left(t_{1}^{\prime}+2 \frac{\left|x_{1}\right|}{v_{F}}\right) s\left(t_{1}\right) s\left(t_{1}+2 \frac{\left|x-x_{1}\right|}{v_{F}}\right)}{s\left(t_{1}-\frac{\left|x_{1}\right|}{v_{F}}+\frac{\left|x-x_{1}\right|}{v_{F}}+i \tau\right) s\left(t_{1}^{\prime}+\frac{\left|x-x_{1}\right|}{v_{F}}+\frac{\left|x_{1}\right|}{v_{F}}+i \tau\right) s\left(t_{1}^{\prime}-\frac{\left|x-x_{1}\right|}{v_{F}}+\frac{\left|x_{1}\right|}{v_{F}}+i \tau\right)}\right]^{\alpha_{e} / 2}, \\
& J_{2 a} \simeq \alpha_{e}^{2}\left(\frac{\pi T}{2}\right)^{2} \int_{0}^{\infty} d t_{1} \int_{0}^{\infty} d t_{1}^{\prime} \frac{1}{s^{2\left(1-\alpha_{e}\right)}\left(t_{1}+t_{1}^{\prime}+\frac{\left|x_{1}\right|}{v_{F}}+\frac{\left|x-x_{1}\right|}{v_{F}}-i \tau\right)}\left[\frac{s\left(t_{1}^{\prime}+\frac{\left|x-x_{1}\right|}{v_{F}}-\frac{\left|x_{1}\right|}{v_{F}}-i \tau\right)}{s\left(t_{1}\right)}\right]^{\alpha_{e} / 2} \\
& \times\left[\frac{s\left(t_{1}^{\prime}+\frac{\left|x-x_{1}\right|}{v_{F}}+\frac{\left|x_{1}\right|}{v_{F}}-i \tau\right) s\left(t_{1}+\frac{\left|x_{1}\right|}{v_{F}}+\frac{\left|x-x_{1}\right|}{v_{F}}-i \tau\right) s\left(t_{1}+\frac{\left|x_{1}\right|}{v_{F}}-\frac{\left|x-x_{1}\right|}{v_{F}}-i \tau\right)}{s\left(t_{1}+2 \frac{\left|x_{1}\right|}{v_{F}}\right) s\left(t_{1}^{\prime}\right) s\left(t_{1}^{\prime}+2 \frac{\left|x-x_{1}\right|}{v_{F}}\right)}\right]^{\alpha_{e} / 2},
\end{aligned}
$$

and $J_{2 b}$ is obtained from $J_{1 a}$ by interchanging $x_{1} \leftrightarrow x-$ $x_{1}$. The terms $J_{1 a}$ and $J_{2 b}$ on one hand and $J_{1 b}$ and $J_{2 a}$ on the other give essentially different contributions to $J\left(x, \tau, x_{1}\right)$. The integrals $J_{1 a}$ and $J_{2 b}$, as a function of the position of the impurity $x_{1}$, are not exponentially small only in a close vicinity of width $\sim u / T$ around $x_{1}=0$ and $x_{1}=x$, respectively. After averaging over $x_{1}$, these "local" terms yield a contribution of order $\mathcal{O}\left(\alpha_{e}^{2}\right)$ which does not depend, in the leading approximation, on $x$ and $\tau$. On the contrary, $J_{1 b}$ and $J_{2 a}$ for small $\alpha_{e}$ do not depend on $x_{1}$ for $x_{1}$ between points $x_{1}=0$ and $x_{1}=x$. The integration over $x_{1}$ for $|x| / v_{F} \gg 1 / T$ yields then the leading contribution to Eq. (E.1). Only the latter terms are thus of importance to us. In the limit of small $\alpha_{e}$, their contribution to $J$ is represented as

$$
\begin{aligned}
& J\left(x, \tau, x_{1}\right) \simeq \alpha_{e}^{2}\left(\frac{\pi T}{2}\right)^{2} \int_{0}^{\infty} d t_{1} \int_{0}^{\infty} d t_{1}^{\prime} \\
& \times\left\{\sinh ^{-2}\left[\pi T\left(t_{1}+t_{1}^{\prime}+\frac{\left|x_{1}\right|}{v_{F}}+\frac{\left|x-x_{1}\right|}{v_{F}}+i \tau\right)\right]\right.
\end{aligned}
$$




$$
+(\tau \rightarrow-\tau)\}
$$

Analytically continuing $J\left(x, \tau, x_{1}\right)$ onto the imaginary axis of $\tau$, we observe that $J\left(x, i t, x_{1}\right)$ is exponentially small for large positive values of $\left(T / v_{F}\right)\left(\left|x_{1}\right|+\left|x-x_{1}\right| \pm\right.$ $\left.v_{F} t\right)$ and is a linear function of this sum when it is large and negative. Since we are interested in the limit of $|x| / v_{F},|t| \gg 1 / T$, we can approximate $J\left(x, i t, x_{1}\right)$ as

$$
\begin{aligned}
& J\left(x, i t, x_{1}\right) \simeq-\frac{\pi}{2} \alpha_{e}^{2} T\left(t-\frac{\left|x_{1}\right|}{v_{F}}-\frac{\left|x-x_{1}\right|}{v_{F}}\right) \\
& \times \Theta\left(-\left|x_{1}\right|-\left|x-x_{1}\right|+v_{F} t\right)+(t \rightarrow-t) .
\end{aligned}
$$

Integrating over $x_{1}$ we obtain

$$
\int d x_{1} I\left(x, i t, x_{1}\right)=-e^{-\left.B_{++}(x, i t)\right|_{\gamma=0}} b(x, i t),
$$

where $b(x, i t)$ is given by Eq. (7.54). We thus provided a direct verification of the RPA form of Eq. (7.8) by deriving the first term in the expansion of $\exp \left(-B_{++}\right)$in powers of $\gamma$ without using the disorder-renormalized effective interaction (7.20). This procedure can be extended to higher powers of $\gamma$ and to the correlator $B_{+-}$.

An important point concerns the factors $\sinh ^{ \pm \alpha_{e} / 2}(\pi T z)$, where $z$ denotes the arguments of the functions $s(z)$ in Eqs. (E.6), (E.7). These were omitted in the limit of small $\alpha_{e}$ in Eq. (E.8). However, even for $\alpha_{e} \ll 1$, this step cannot be justified for arbitrary $x, x_{1}$, and $\tau$, e.g., if $\alpha_{e}|x| T / v_{F} \gg 1$. What is important to us is that all these factors actually cancel each other after the analytical continuation onto the imaginary axis of $\tau \rightarrow i t$ for large positive values of $-\left|x_{1}\right|-\left|x-x_{1}\right| \pm u t$. As Eq. (E.9) shows, only this range of $t$ is relevant to $J\left(x, i t, x_{1}\right)$, which is the quantity used in the calculation of the dephasing rate. The RPA for pairing of all four fields at points $(0,0)$ and $(x, \tau)$, shown in Fig. 24, namely of the combinations $\theta_{+}(x, \tau)-\theta_{-}(x, \tau)$ and $\theta_{+}(0,0)-\theta_{-}(0,0)$ with each other, via the fermionic bubble with two backscatterings at $x=x_{1}$ can be reproduced in a similar way.

\section{APPENDIX F: CLASSICAL TRAJECTORY FOR THE COOPERON}

In this Appendix, we substantiate an important step in our derivation of the interaction-induced action $S_{C}$ of the Cooperon within the functional-bosonization scheme. Specifically, we analyze the role of the factors $\eta_{ \pm}(x, \tau)$, Eq. (7.13), which contain both the Fermi velocity $v_{F}$ and the plasmon velocity $u$. We demonstrate that in the spinless case, considered in this paper, the velocity of electrons on the "Cooperon path" which contributes to $\Delta \sigma_{\mathrm{wl}}$ is given by $u$ and not by $v_{F}$. Put another way, the velocity of the interfering quasiclassical trajectories is renormalized by the interaction and coincides with that of the RPA propagators. As a result, one can omit the potentially dangerous factors $\eta_{ \pm}(x, \tau)$ in the calculation of the WL dephasing.

The function $c_{3}$ in Eq. (7.61), which gives the threeimpurity Cooperon loop, is explicitely written as

$$
\begin{aligned}
& c_{3}\left(x, \tau_{f}-\tau_{i}, x_{1}, x_{2}, x_{3}\right)=\left(\frac{v_{F} \gamma_{0}}{2}\right)^{3} \\
& \times \int_{0}^{1 / T} d \tau_{1} \int_{0}^{1 / T} d \bar{\tau}_{1} \int_{0}^{1 / T} d \tau_{2} \int_{0}^{1 / T} d \bar{\tau}_{2} \int_{0}^{1 / T} d \tau_{3} \int_{0}^{1 / T} d \bar{\tau}_{3} \\
& \times\left\langle g_{+}^{(0)}\left(x_{1}, \tau_{1}-\tau_{i}\right) e^{i\left[\theta_{+}\left(x_{1}, \tau_{1}\right)-\theta_{-}\left(x_{1}, \tau_{1}\right)\right]}\right. \\
& \times \quad g_{-}^{(0)}\left(x_{2}-x_{1}, \tau_{2}-\tau_{1}\right) e^{-i\left[\theta_{+}\left(x_{2}, \tau_{2}\right)-\theta_{-}\left(x_{2}, \tau_{2}\right)\right]} \\
& \times \quad g_{+}^{(0)}\left(x_{3}-x_{2}, \tau_{3}-\tau_{2}\right) e^{i\left[\theta_{+}\left(x_{3}, \tau_{3}\right)-\theta_{-}\left(x_{3}, \tau_{3}\right)\right]} \\
& \times \quad g_{-}^{(0)}\left(x-x_{3}, \tau_{f}-\tau_{3}\right) \\
& \times \quad g_{-}^{(0)}\left(x_{1}-x, \bar{\tau}_{1}-\tau_{f}\right) e^{-i\left[\theta_{+}\left(x_{1}, \bar{\tau}_{1}\right)-\theta_{-}\left(x_{1}, \bar{\tau}_{1}\right)\right]} \\
& \times \quad g_{+}^{(0)}\left(x_{2}-x_{1}, \bar{\tau}_{2}-\bar{\tau}_{1}\right) e^{i\left[\theta_{+}\left(x_{2}, \bar{\tau}_{2}\right)-\theta_{-}\left(x_{2}, \bar{\tau}_{2}\right)\right]} \\
& \times \quad g_{-}^{(0)}\left(x_{3}-x_{2}, \bar{\tau}_{3}-\bar{\tau}_{2}\right) e^{-i\left[\theta_{+}\left(x_{3}, \bar{\tau}_{3}\right)-\theta_{-}\left(x_{3}, \bar{\tau}_{3}\right)\right]} \\
& \left.\times \quad g_{+}^{(0)}\left(-x_{3}, \tau_{i}-\bar{\tau}_{3}\right)\right\rangle,
\end{aligned}
$$

where we write the external times of the bubble as $\tau_{i}$ and $\tau_{f}$ [in Eq. (7.61), $\tau_{f}-\tau_{i}=\tau$ ]. To examine the role of the factors $\eta_{ \pm}$, it is convenient to introduce the function

$$
C_{3}=\int_{0}^{1 / T} d \tau_{i} \int_{0}^{1 / T} d \tau_{f} c_{3} e^{i \Omega_{n}\left(\tau_{f}-\tau_{i}\right)}
$$

by integrating first over the external times.

The averaging $\langle\ldots\rangle$ over the fluctuating fields $\theta_{ \pm}(x, \tau)$ yields the factor

$$
\exp \left[-2\left(M_{f f}-M_{f b}\right)\right],
$$

defined in Eqs. (7.37) (7.39), in the integrand of Eq. (F.1). In the absence of interaction $\left(M_{f f}=M_{f b}=\right.$ $0)$, the integrals over the internal times are determined by the poles of the free Green's functions [specified by Eq. (F.4) below with $u \rightarrow v_{F}$ ]. For given coordinates $x_{1}$, $x_{2}, x_{3}$, and $x$, we refer to this set of times as the "classical trajectory" of the Cooperon with velocity $v_{F}$.

Each interaction-induced term $M\left(N, N^{\prime}\right)$ in Eqs. (7.37), (7.38) contains the combination $B_{++}+B_{--}$, and hence depends on both $u$ and $v_{F}$. If one neglects disorder in the RPA propagators ("clean RPA"), the factor $\exp \left[-M\left(N, N^{\prime}\right)\right]$ is represented as

$$
\begin{aligned}
e^{-M\left(N, N^{\prime}\right)} & =e^{-\alpha_{e} \mathcal{L}\left(x_{N}-x_{N^{\prime}}, \tau_{N}-\tau_{N^{\prime}}\right)} \\
& \times \zeta\left(x_{N}-x_{N^{\prime}}, \tau_{N}-\tau_{N^{\prime}}\right),
\end{aligned}
$$

where $\zeta(x, \tau)=\eta_{+}(x, \tau) \eta_{-}(x, \tau), \quad \alpha_{e}=1-K$, and the functions $\mathcal{L}(x, \tau)$ and $\eta_{ \pm}(x, \tau)$ are given by Eqs. (7.12), (17.13). The dependence on $v_{F}$ comes from the factors $\eta_{ \pm}$. The "dirty RPA" does not affect the latter. We thus see that each pair of impurities $N$ and $N^{\prime}$ in Eq. (F.1) generates two poles associated with the 
functions $\eta_{ \pm}$:

$$
\begin{aligned}
& \zeta(x, \tau)=\left(\frac{v_{F}}{u}\right)^{2} \\
& \times \frac{\sinh \left[\pi T\left(x / v_{F}+i \tau\right)\right] \sinh \left[\pi T\left(x / v_{F}-i \tau\right)\right]}{\sinh [\pi T(x / u+i \tau)] \sinh [\pi T(x / u-i \tau)]} .
\end{aligned}
$$

The singularities in Eq. (F.1) coming from the functions $\zeta(x, \tau)$ are analogous to those in the bare Green's functions $g_{ \pm}^{(0)}(x, \tau)$. However, they can appear either as " $u$ poles" at $i \tau= \pm x / u$ or as " $v_{F}$-poles" at $i \tau= \pm x / v_{F}$, depending on the sign of the factor $(-1)^{N+N^{\prime}}$ or $(-1)^{N+\bar{N}}$ in Eqs. (7.37), (7.38).

We can readily integrate over $\tau_{i}$ and $\tau_{f}$ the free Green's functions that connect to the external vertices. Since there are no interaction-induced factors at the external vertices, the integration yields again the free Green's functions. The function $C_{3}$ then becomes

$$
\begin{aligned}
& C_{3}=\left(\frac{v_{F} \gamma_{0}}{2}\right)^{3} \frac{1}{v_{F}^{2}} \\
& \times \int_{0}^{1 / T} d \tau_{1} \int_{0}^{1 / T} d \bar{\tau}_{1} \int_{0}^{1 / T} d \tau_{2} \int_{0}^{1 / T} d \bar{\tau}_{2} \int_{0}^{1 / T} d \tau_{3} \int_{0}^{1 / T} d \bar{\tau}_{3} \\
& \times \quad g_{+}^{(0)}\left(x_{1}-x_{3}, \tau_{1}-\bar{\tau}_{3}\right) \zeta\left(x_{1}-x_{3}, \tau_{1}-\bar{\tau}_{3}\right) \\
& \times \quad g_{-}^{(0)}\left(x_{2}-x_{1}, \tau_{2}-\tau_{1}\right) \zeta\left(x_{2}-x_{1}, \tau_{2}-\tau_{1}\right) \\
& \times \quad g_{+}^{(0)}\left(x_{3}-x_{2}, \tau_{3}-\tau_{2}\right) \zeta\left(x_{3}-x_{2}, \tau_{3}-\tau_{2}\right) \\
& \times \quad g_{-}^{(0)}\left(x_{1}-x_{3}, \bar{\tau}_{1}-\tau_{3}\right) \zeta\left(x_{1}-x_{3}, \bar{\tau}_{1}-\tau_{3}\right) \\
& \times \quad g_{+}^{(0)}\left(x_{2}-x_{1}, \bar{\tau}_{2}-\bar{\tau}_{1}\right) \zeta\left(x_{2}-x_{1}, \bar{\tau}_{2}-\bar{\tau}_{1}\right) \\
& \times \quad g_{-}^{(0)}\left(x_{3}-x_{2}, \bar{\tau}_{3}-\bar{\tau}_{2}\right) \zeta\left(x_{3}-x_{2}, \bar{\tau}_{3}-\bar{\tau}_{2}\right) \\
& \times \quad \zeta^{-1}\left(x_{1}-x_{3}, \tau_{1}-\tau_{3}\right) \zeta^{-1}\left(x_{1}-x_{3}, \bar{\tau}_{1}-\bar{\tau}_{3}\right) \\
& \times \quad \zeta^{-1}\left(x_{1}-x_{2}, \tau_{1}-\bar{\tau}_{2}\right) \zeta^{-1}\left(x_{3}-x_{2}, \tau_{3}-\bar{\tau}_{2}\right) \\
& \times \quad \zeta^{-1}\left(x_{2}-x_{1}, \tau_{2}-\bar{\tau}_{1}\right) \zeta^{-1}\left(x_{2}-x_{3}, \tau_{2}-\bar{\tau}_{3}\right) \\
& \times \quad \zeta\left(0, \tau_{1}-\bar{\tau}_{1}\right) \zeta\left(0, \tau_{2}-\bar{\tau}_{2}\right) \zeta\left(0, \tau_{3}-\bar{\tau}_{3}\right) \\
& \times \quad R\left(x_{1}, x_{2}, x_{3}, \tau_{1}, \tau_{2}, \tau_{3}, \bar{\tau}_{1}, \bar{\tau}_{2}, \bar{\tau}_{3}\right) \\
& \times \quad W\left(x_{1}, \tau_{1}, x_{3}, \bar{\tau}_{3}, \Omega_{n}\right) \\
& \times \quad W\left(x-x_{3}, \tau_{3}, x-x_{1}, \bar{\tau}_{1},-\Omega_{n}\right), \\
& \times \quad x,
\end{aligned}
$$

where $\zeta(0, \tau)=\left(v_{F} / u\right)^{2}$, the function $R$ combines all the factors coming from the first factor in Eq. (F.2), and

$$
\begin{aligned}
& W\left(x_{1}, \tau_{1}, x_{3}, \bar{\tau}_{3}, \Omega_{n}\right) \\
= & e^{-\left|\Omega_{n} x_{1}\right|-i \Omega_{n} \tau_{1}}\left[\Theta\left(\Omega_{n}\right) \Theta\left(x_{1}\right)-\Theta\left(-\Omega_{n}\right) \Theta\left(-x_{1}\right)\right] \\
+ & e^{-\left|\Omega_{n} x_{3}\right|-i \Omega_{n} \bar{\tau}_{1}}\left[\Theta\left(\Omega_{n}\right) \Theta\left(x_{3}\right)-\Theta\left(-\Omega_{n}\right) \Theta\left(-x_{3}\right)\right] .
\end{aligned}
$$

In the single-particle Green's function, the Fermi velocity $v_{F}$ drops out completely in the product $g_{ \pm}^{(0)}(x, \tau) \eta_{ \pm}(x, \tau)$. One can see that each free Green's function in Eq. (F.3) is multiplied by a corresponding factor $\eta_{ \pm}$, so that all the $v_{F}$-poles associated with the free Green's functions in Eq. F.3 are replaced by $u$ poles. One might think that $v_{F}$ disappears completely from the problem, and hence the integration over the internal times in Eq. (F.3) straightforwardly yields a new classical trajectory defined by

$$
\begin{aligned}
i \tau_{1} & =-x_{1} / u, \\
i \tau_{2} & =\left(x_{2}-2 x_{1}\right) / u, \\
i \tau_{3} & =\left(2 x_{2}-2 x_{1}-x_{3}\right) / u, \\
i \bar{\tau}_{3} & =-x_{3} / u, \\
i \bar{\tau}_{2} & =\left(x_{2}-2 x_{3}\right) / u, \\
i \bar{\tau}_{1} & =\left(2 x_{2}-2 x_{3}-x_{1}\right) / u
\end{aligned}
$$

However, the impurity vertices are all interconnected by the interaction propagators. Among the latter, there are propagators that connect vertices which are not connected by the single-particle Green's functions [e.g., points $\left(x_{1}, \tau_{1}\right)$ and $\left.\left(x_{2}, \overline{\tau_{2}}\right)\right]$. As a result, the $v_{F}$-poles in the corresponding functions $\eta_{ \pm}$remain uncompensated.

In the noninteracting case, the sequence of points in real space

$$
1 \rightarrow 2 \rightarrow 3 \rightarrow 1 \rightarrow 2 \rightarrow 3 \rightarrow 1
$$

forms a closed loop with segments given by the Green's functions $g_{ \pm}^{(0)}$. Now that we have the interaction-induced functions $\eta_{ \pm}$that connect every pair of impurities, other trajectories can contribute to $C_{3}$, whose segments are given either by the Green's function or by the function $\eta_{ \pm}$. In view of this, the notion of a single classical trajectory contributing to $\Delta \sigma_{\mathrm{wl}}$ requires a more accurate justification. As we show below, the interaction itself "chooses" a unique classical trajectory which is defined in Eq. (F.4 , i.e., the one that is determined by a combination of the Green's functions with the renormalized velocity $v_{F} \rightarrow u$. The contributions of all other trajectories are exponentially suppressed. Note that within the fermionic path-integral approach used in Sec. VI, the "correct" velocity is chosen automatically on the saddlepoint trajectory of the total action.

When analyzing the role of the factors $\eta_{ \pm}$, we can, in the first approximation, neglect other interactioninduced factors in Eq. F.1 by setting the exponent $\alpha_{e}=0$ in Eq. (F.2), i.e., by putting $R=1$ in Eq. (F.3) while keeping the difference between $v_{F}$ and $u$ in the functions $\zeta$. This simplifies the analytical structure of the integrand in Eq. (F.3) by reducing the integration along branch cuts to the integration around simple poles only. The integration around all the poles then yields $C_{3}$ represented as a sum over a whole set of trajectories, not only the one given by Eq. (F.5).

The integrand of Eq. (F.3) contains products of the type $g_{ \pm}^{(0)}(x, i t) \zeta(x, i t)$ which, as a function of $t$, have poles on the classical trajectory (F.4) at $t= \pm x / u$ with a residue equal to

$$
\begin{aligned}
& {\left.[u t \mp(x+u \operatorname{sgn} x / \Lambda)] g_{ \pm}^{(0)}(x, i t) \zeta(x, i t)\right|_{t \rightarrow \pm x / u}} \\
& \simeq-\frac{1}{2 \pi i} \frac{v_{F}}{u} \exp \left(\frac{u-v_{F}}{u v_{F}} \pi T|x|\right)
\end{aligned}
$$


for $|x| / u \gg 1 / T$. Writing the remaining factors $\zeta$ which are not singular at this pole as

$$
\begin{aligned}
\zeta(x, i t) & \simeq\left(\frac{v_{F}}{u}\right)^{2} \exp \left[\pi T\left(\left|t_{+}^{v}\right|+\left|t_{-}^{v}\right|-\left|t_{+}^{u}\right|-\left|t_{-}^{u}\right|\right)\right] \\
& \times \operatorname{sgn} t_{+}^{v} \operatorname{sgn} t_{-}^{v} \operatorname{sgn} t_{+}^{u} \operatorname{sgn} t_{-}^{u}
\end{aligned}
$$

for $\left|t_{ \pm}^{v, u}\right| \gg 1 / T$, where $t_{ \pm}^{v}=t \pm x / v$ and $t_{ \pm}^{u}=t \pm x / u$, we observe that all the exponential factors coming from Eqs. (F.6), (F.7) cancel out on the classical trajectory (F.4).

On the contrary, any other sequence of poles has at least some of the segments of the trajectory passed with velocity $v_{F}$ and no such compensation occurs, which leads to an exponential decay of the contribution to $C_{3}$. The exponent depends on a particular sequence of poles but for any sequence is at least of the order of
$\left(\left|u-v_{F}\right| / v_{F}\right) T t_{c}$. One sees that the contribution of any of the "nonclassical" trajectories is exponentially suppressed either by the "Golden-Rule dephasing" or simply by the thermal smearing. The role of the factors $\eta_{ \pm}$ is thus two-fold: they renormalize the velocity on the "classical" Cooperon trajectory $\left(v_{F} \rightarrow u\right)$ and suppress the contribution of all nonclassical trajectories. Taking into account the factor $R$ in Eq. (F.3) within the "clean RPA" does not give any decay on this classical trajectory either. Including disorder within the "dirty RPA" (Sec. VIIC and Appendix E) in the factor $R$ yields an exponential decay on the classical trajectory as well, as discussed in Sec. VIIF but this decay is much weaker than on the nonclassical trajectories, so that the latter can indeed be neglected in the calculation of $\Delta \sigma_{\mathrm{wl}}$.
* Also at A.F. Ioffe Physico-Technical Institute, 194021 St. Petersburg, Russia.

$\dagger$ Also at Petersburg Nuclear Physics Institute, 188350 St. Petersburg, Russia.

1 Carbon Nanotubes, Special Issue of Physics World (June 2000).

2 O.M. Auslaender, A. Yacoby, R. de Picciotto, K.W. Baldwin, L.N. Pfeiffer, and K.W. West, Phys. Rev. Lett. 84, 1764 (2000); Science 295, 825 (2002); O.M. Auslaender, H. Steinberg, A. Yacoby, Y. Tserkovnyak, B.I. Halperin, K.W. Baldwin, L.N. Pfeiffer, and K.W. West, ibid. 308, 88 (2005).

3 E. Levy, A. Tsukernik, M. Karpovski, A. Palevski, B. Dwir, E. Pelucchi, A. Rudra, E. Kapon, and Y. Oreg, cond-mat/0509027

4 S.V. Zaitsev-Zotov, Y.A. Kumzerov, Y.A. Firsov, and P. Monceau, J. Phys. Cond. Matter 12, L303 (2000); Pis'ma v ZhETF 77, 162 (2003) [JETP Lett. 77, 135 (2003)].

5 W. Kang, H.L. Stormer, L.N. Pfeiffer, K.W. Baldwin, and K.W. West, Nature 403, 59 (2000); I. Yang, W. Kang, K.W. Baldwin, L.N. Pfeiffer, and K.W. West, Phys. Rev. Lett. 92, 056802 (2004).

6 M. Grayson, D. Schuh, M. Huber, M. Bichler, and G. Abstreiter, Appl. Phys. Lett. 86, 032101 (2005) and to be published.

7 A.N. Aleshin, H.J. Lee, Y.W. Park, and K. Akagi, Phys. Rev. Lett. 93, 196601 (2004); A.N. Aleshin, Adv. Mat. 18, 17 (2006) and references therein.

8 E. Slot, M.A. Holst, H.S.J. van der Zant, and S.V. ZaitsevZotov, Phys. Rev. Lett. 93, 176602 (2004).

9 L. Venkataraman, Y.S. Hong, and P. Kim, Phys. Rev. Lett. 96, 076601 (2006).

10 M. Bockrath, D.H. Cobden, J. Lu, A.G. Rinzler, R.E. Smalley, L. Balents, and P.L. McEuen, Nature 397, 598 (1999).

11 Z. Yao, H. Postma, L. Balents, and C. Dekker, Nature 402, 273 (1999).

12 Z. Yao, C.L. Kane, and C. Dekker, Phys. Rev. Lett. 84, 2941 (2000).

13 H.R. Shea, R. Martel, and Ph. Avouris, Phys. Rev. Lett.
84, $4441(2000)$.

14 J. Nygård, D.H. Cobden, and P.E. Lindelof, Nature 408, $342(2000)$.

15 M. Bockrath, W. Liang, D. Bozovic, J.H. Hafner, C.M. Lieber, M. Tinkham, and H. Park, Science 291, 283 (2001)

16 H.W.Ch. Postma, T. Teepen, Z. Yao, M. Grifoni, and C. Dekker, Science 293, 76 (2001).

17 R. Krupke, F. Hennrich, H.B. Weber, D. Beckmann, O. Hampe, S. Malik, M.M. Kappes, and H. v. Löhneysen, Appl. Phys. A 76, 397 (2003).

18 N. Mason, M.J. Biercuk, and C.M. Marcus, Science 303, 655 (2004).

19 H.T. Man and A.F. Morpurgo, Phys. Rev. Lett. 95, 026801 (2005).

20 J. Wei, M. Shimogawa, Z. Wang, I. Radu, R. Dormaier, and D.H. Cobden, Phys. Rev. Lett. 95, 256601 (2005).

21 P.J. Leek, M.R. Buitelaar, V.I. Talyanskii, C.G. Smith, D. Anderson, G.A.C. Jones, J. Wei, and D.H. Cobden, Phys. Rev. Lett. 95, 256802 (2005).

22 H.T. Man, I.J.W. Wever, and A.F. Morpurgo, Phys. Rev. B 73, 241401 (2006).

23 A.Y. Kasumov, R. Deblock, M. Kociak, B. Reulet, H. Bouchiat, I.I. Khodos, Yu.B. Gorbatov, V.T. Volkov, C. Journet, and M. Burghard, Science 284, 1508 (1999).

24 A.F. Morpurgo, J. Kong, C.M. Marcus, and H. Dai, Science 286, 263 (1999).

25 P. Jarillo-Herrero, J.A. van Dam, and L.P. Kouwenhoven, Nature 439, 953 (2006).

26 S. Li, Z. Yu, C. Rutherglen, and P.J. Burke, Nano Letters, 4, 2003 (2004).

27 J. Sólyom, Adv. Phys. 28, 201 (1979).

28 J. Voit, Rep. Prog. Phys. 57, 977 (1994).

29 H.J. Schulz, in Mesoscopic Quantum Physics, edited by E. Akkermans, G. Montambaux, J.-L. Pichard, and J. Zinn-Justin (North-Holland, Amsterdam, 1995).

30 A.O. Gogolin, A.A. Nersesyan, and A.M. Tsvelik, Bosonization and Strongly Correlated Systems (Cambridge University Press, Cambridge, 1998).

31 H.J. Schulz, G. Cuniberti, and P. Pieri, in Field Theories for Low-Dimensional Condensed Matter Systems, 
edited by G. Morandi, P. Sodano, P. Tagliacozzo, and V. Tognetti (Springer, Berlin, 2000)

32 T. Giamarchi, Quantum Physics in One Dimension (Oxford University Press, Oxford, 2004).

33 W. Apel and T.M. Rice, J. Phys. C 16, L271 (1982); Phys. Rev. B 26, 7063 (1982).

34 T. Giamarchi and H.J. Schulz, Phys. Rev. B 37, 325 (1988).

35 M.-R. Li and E. Orignac, Europhys. Lett. 60, 432 (2002).

36 T. Giamarchi, T. Nattermann, and P. Le Doussal in Fundamental Problems of Mesoscopic Physics, edited by I.V. Lerner, B.L. Altshuler, and Y. Gefen (Kluwer, Dordrecht, 2004).

37 T. Nattermann, T. Giamarchi, and P. Le Doussal, Phys. Rev. Lett. 91, 056603 (2003).

38 S.V. Malinin, T. Nattermann, and B. Rosenow, Phys. Rev. B 70, 235120 (2004).

39 A. Glatz and T. Nattermann, Phys. Rev. B 69, 115118 (2004).

40 S.N. Artemenko and S.V. Remizov, Phys. Rev. B 72, 125118 (2005); S.N. Artemenko, J. de Physique IV 131, 175 (2005).

41 A.L. Chudnovskiy, Phys. Rev. B 72, 195302 (2005).

42 B. Rosenow and T. Nattermann, Phys. Rev. B 73, 085103 (2006).

43 C. Mora, R. Egger, and A. Altland, cond-mat/0602411.

44 A.G. Yashenkin, I.V. Gornyi, A.D. Mirlin, and D.G. Polyakov, in preparation.

45 I.V. Gornyi, A.D. Mirlin, and D.G. Polyakov, Phys. Rev. Lett. 95, 046404 (2005).

46 B.L. Altshuler and A.G. Aronov, in Electron-Electron Interactions in Disordered Systems, edited by A.L. Efros and M. Pollak (North-Holland, Amsterdam, 1985).

47 Mesoscopic Phenomena in Solids, edited B.L. Altshuler, P.A. Lee, and R.A. Webb (North-Holland, Amsterdam, 1991).

48 J. Imry, Introduction to Mesoscopic Physics (Oxford University Press, Oxford, 1997).

49 K.B. Efetov, Supersymmetry in Disorder and Chaos (Cambridge University Press, Cambridge, 1997).

50 I.L. Aleiner, B.L. Altshuler, and M.E. Gershenson, Waves in Random Media 9, 201 (1999).

51 A.D. Mirlin, Phys. Rep. 326, 259 (2000).

52 L.P. Gorkov, A.I. Larkin, and D.E. Khmelnitskii, Pis'ma v ZhETF 30, 248 (1979) [JETP Lett. 30, 228 (1979)].

53 A.M. Rudin, I.L. Aleiner, and L.I. Glazman, Phys. Rev. B 55, 9322 (1997).

54 G. Zala, B.N. Narozhny, and I.L. Aleiner, Phys. Rev. B 64, 214204 (2001).

55 A.M. Finkel'stein, Zh. Éksp. Teor. Fiz. 84, 168 (1983) [Sov. Phys. JETP 57, 97 (1983)]; Z. Phys. B: Condens. Matter 56, 189 (1984); in Electron Liquid in Disordered Conductors, edited by I.M. Khalatnikov, Sov. Sci. Rev. A. Phys. 14, 1 (1990).

56 L.S. Levitov and A.V. Shytov, Pis'ma v ZhETF 66, 200 (1997) [JETP Lett. 66, 214 (1997)].

57 A. Kamenev and A. Andreev, Phys. Rev. B 60, 2218 (1999).

58 B.L. Altshuler, A.G. Aronov, and D.E. Khmelnitskii, J. Phys. C 15, 7367 (1982).

59 P.W. Anderson, Phys. Rev. 109, 1492 (1958).

60 E. Abrahams, P.W. Anderson, D.C. Licciardello, and T.V. Ramakrishnan, Phys. Rev. Lett. 42, 673 (1979).
61 N. Mott, Metal-Insulator-Transitions (Taylor \& Francis, London, 1974).

62 B.I. Shklovskii and A.L. Efros, Electronic Properties of Doped Semiconductors (Springer, Berlin, 1984); A.L. Efros and B.I. Shklovskii, in Electron-Electron Interaction in Disordered Systems, edited by A.L. Efros and M. Pollak, (North-Holland, Amsterdam, 1985).

63 L. Fleishman, D.C. Licciardello, and P.W. Anderson, Phys. Rev. Lett. 40, 1340 (1978).

64 L. Fleishman and P.W. Anderson, Phys. Rev. B 21, 2366 (1980).

65 I.V. Gornyi, A.D. Mirlin, and D.G. Polyakov, Phys. Rev. Lett. 95, 206603 (2005)

66 D.M. Basko, I.L. Aleiner, and B.L. Altshuler, Ann. Phys. (N.Y.) 321, 1126 (2006).

67 M.E. Gertsenshtein and V.B. Vasil'ev, Radiotekhnika i Elektronika 4, 611 (1959); Teor. Veryatn. Primen. 4, 424 (1959) [Theor. Probab. Appl. 4, 391 (1959)].

68 N.F. Mott and W.D. Twose, Adv. Phys. 10, 107 (1961); R.E. Borland, Proc. Phys. Soc. London 78, 926 (1961); Proc. R. Soc. London, Ser. A 274, 529 (1963).

69 V.L. Berezinskii, Zh. Éksp. Teor. Fiz. 65, 1251 (1973) [Sov. Phys. JETP 38, 620 (1974)].

70 A.A. Gogolin, V.I. Mel'nikov, and É.I. Rashba, Zh. Éksp. Teor. Fiz. 69, 327 (1975) [Sov. Phys. JETP 42, 168 (1975)].

71 A.A. Abrikosov and I.A. Ryzhkin, Adv. Phys. 27, 147 (1978).

72 D.J. Thouless, Phys. Rev. Lett. 39, 1167 (1977).

73 V.L. Berezinskii and L.P. Gor'kov, Zh. Éksp. Teor. Fiz. 77, 2498 (1979) [Sov. Phys. JETP 50, 1209 (1979)].

74 A.A. Gogolin, Phys. Rep. 86, 1 (1982).

75 I.M. Lifshitz, S.A. Gredeskul, and L.A Pastur, Introduction to the Theory of Disordered Systems (New York, Wiley, 1988).

76 L.P. Gor'kov and I.E. Dzyaloshinskii, Pis'ma v ZhETF 18, 686 (1973) [JETP Lett. 18, 401 (1973)].

77 D.C. Mattis, Phys. Rev. Lett. 32, 714 (1974).

78 A. Luther and I. Peschel, Phys. Rev. Lett. 32, 992 (1974).

79 K.A. Matveev, D. Yue, and L.I. Glazman, Phys. Rev. Lett. 71, 3351 (1993); D. Yue, L.I. Glazman, and K.A. Matveev, Phys. Rev. B 49, 1966 (1994).

${ }^{80}$ C.L. Kane and M.P.A. Fisher, Phys. Rev. Lett. 68, 1220 (1992); Phys. Rev. B 46, 15233 (1992).

81 A. Furusaki and N. Nagaosa, Phys. Rev. B 47, 4631 (1993).

82 S.T. Chui and J.W. Bray, Phys. Rev. B 16, 1329 (1977).

${ }^{83}$ Y. Suzumura and H. Fukuyama, J. Phys. Soc. Jpn. 52, 2870 (1983); ibid. 53, 3918 ( 1984).

84 P.W. Anderson, D.J. Thouless, E. Abrahams, and D.S. Fisher, Phys. Rev. B 22, 3519 (1980).

85 A. Furusaki and N. Nagaosa, Phys. Rev. B 47, 3827 (1993).

86 D.L. Maslov, Phys. Rev. B 52, R14368 (1995).

87 N.P. Sandler and D.L. Maslov, Phys. Rev. B 5513808 (1997).

88 D.L. Maslov, Fundamental aspects of electron correlations and quantum transport in one-dimensional systems, Lecture course at the LXXXI Les Houches School on Nanoscopic Quantum Transport (2004); cond-mat/0506035.

89 H.C. Fogedby, J. Phys. C 9, 3757 (1976).

90 D.K.K. Lee and Y. Chen, J. Phys. A 21, 4155 (1988). 
91 P. Kopietz, Bosonization of Interacting Electrons in Arbitrary Dimensions (Springer, Berlin, 1997).

92 I.V. Yurkevich, in Strongly Correlated Fermions and Bosons in Low-Dimensional Disordered Systems, edited by I.V. Lerner, B.L. Altshuler, and V.I. Fal'ko (Kluwer, Dordrecht, 2002), cond-mat/0112270

93 I.V. Lerner and I.V. Yurkevich, Impurity in the Tomonaga-Luttinger model: a functional integral approach, to be published in Proc. LXXXI Les Houches School on Nanoscopic Quantum Transport (2004); cond-mat/0508223.

94 See, e.g., D.G. Polyakov and I.V. Gornyi, Phys. Rev. B 68, 035421 (2003) and references therein.

95 A. Kawabata, J. Phys. Soc. Japan 65, 30 (1996).

96 Y. Oreg and A.M. Finkelstein, Phys. Rev. B 54, 14265 (1996).

97 T. Giamarchi, Phys. Rev. B 44, 2905 (1991).

98 T. Giamarchi, Ph.D. Thesis (1987, unpublished).

99 B.N. Narozhny, G. Zala, and I.L. Aleiner, Phys. Rev. B 65, 180202 (2002).

100 K. Le Hur, Phys. Rev. B 65, 233314 (2002).

101 More generally, this applies also to a Cooperon ladder with an arbitrary even number of backscattering impurity lines.

102 V.M. Gasparyan and A.Yu. Zyuzin, Fiz. Tverd. Tela 27, 1662 (1985) [Sov. Phys. Solid State 27, 999 (1985)]; A.P. Dmitriev, V.Yu. Kachorovskii, and I.V. Gornyi, Phys. Rev. B 56, 9910 (1997).

103 Strictly speaking, this is true for a linear dispersion only. In reality, a finite curvature $m^{-1}$ will lead to an additional contribution to the dephasing rate $\sim \alpha^{2} T^{2} / m v_{F}^{2}$.
One sees, however, that Eq. (6.14) gives the leading contribution in a wide temperature range $T \ll\left(m^{2} v_{F}^{4} / \alpha^{2} \tau\right)^{1 / 3}$.

104 A.A. Gogolin and G.T. Zimanyi, Solid State Comm. 46, 469 (1983).

105 A similar transport regime in the context of a dynamical localization was found in D.M. Basko, Phys. Rev. Lett. 91, 206801 (2003).

106 K. Le Hur, Phys. Rev. Lett. 95, 076801 (2005); cond-mat/0606387.

107 G. Seelig and M. Büttiker, Phys. Rev. B 64, 245313 (2001); G. Seelig, S. Pilgram, A.N. Jordan, and M. Büttiker, ibid. 68, 161310 (2003).

108 T. Ludwig and A.D. Mirlin, Phys. Rev. B 69, 193306 (2004).

109 V. Fernández, K. Li, and C. Naón, Phys. Lett. B 452, 98 (1999); V.I. Fernández and C.M. Naón, Phys. Rev. B 64, 033402 (2001).

110 A. Grishin, I.V. Yurkevich, and I.V. Lerner, Phys. Rev. B 69, 165108 (2004).

111 I.E. Dzyaloshinskii and A.I. Larkin, Zh. Éksp. Teor. Fiz. 65, 411 (1973) [Sov. Phys. JETP 38, 202 (1974)].

112 Note that if we were to start with a quadratic dispersion and then linearize, an additional term in the current vertices would appear proportional to $\pm \partial_{\tau} \theta_{ \pm}$, as pointed out in Ref. 93. This, however, only yields an ultraviolet renormalization of the bare Fermi velocity $v_{F}$ by e-e interactions with high-energy transfers. In this sense, in our model $v_{F}$ already includes all "Fermi-liquid-like" renormalizations of the electron spectrum. 